\title{
Designing Practical and Fair Sequential Team Contests*
}

\author{
Nejat Anbarc1 ${ }^{\dagger} \quad$ Ching-Jen Sun ${ }^{\ddagger} \quad$ M. Utku Ünver ${ }^{\S}$
}

This Draft: April 2021

\begin{abstract}
Economists have long recognized that the effect of the order of actions in sequential contests on performance of the contestants is far from negligible. We model the tiebreak mechanisms, known as penalty shootouts, which have sequential move order and are used in several teamsports contests, as a practical dynamic mechanism-design problem. We characterize all orderindependent mechanisms; in such mechanisms two balanced teams have equal chances to win the shootout whenever the score is tied after equal numbers of attempts and hence move order has no relevance for winning chances. Using additional desirable properties, we uniquely characterize practical mechanisms. In most sports, such as football and hockey, the order in which teams take the penalties is fixed, known as ABAB, and a few high-level football competitions recently adopted the alternating-order variant mechanism, ABBA. Our results imply that these two and all other exogenous-order mechanisms - with one exception - are order dependent in regular rounds. Although ABBA is order independent in sudden-death rounds, ABAB fails there, too. Our theory supports empirical studies linking ABAB to unfair outcomes and multiple equilibria in terms of winning chances of the first- vs. second-kicking teams in different football traditions.
\end{abstract}

Keywords: Tiebreak mechanisms, penalty shootouts, fairness in sequential contests, mechanism design, market design, order independence

JEL Codes: D78, D63, D47, C79

${ }^{*}$ We would like to thank J. Apesteguia, G. Artemov, T. Bergstrom, S. Brams, N. Feltovich, S. Grant, R. Holden, M. Jackson, J. Kline, L. Lenten, S. Loertscher, I. MacKenzie, P. Man, F. Massari, A. McLennan, C. Mezzetti, S. Özertürk, I. Palacios-Huerta, M. Pycia, R. Silvers, T. Sönmez, W. Thomson, J. Wooders, Ü. Zenginobuz, and the audiences at ASSET, Australasian Econ Theory, NBER Decentralization, SSCW, Stony Brook Game Theory, Monash Market Design, and SfED conference presentations and various university seminars for their comments. This draft benefited from many constructive comments of an anonymous advisory editor and two referees of the journal.

${ }^{\dagger}$ Professor, Durham University, Department of Economics and Finance; Professorial Research Fellow, Deakin University, Department of Economics; nejat.anbarci@durham.ac.uk

${ }^{\ddagger}$ Associate Professor, Deakin University, Department of Economics; ching-jen.sun@deakin.edu.au

$\S$ Professor, Boston College, Department of Economics; Professorial Research Fellow, Deakin University, Department of Economics; unver@bc.edu 


\section{Introduction}

In this paper, we model shootouts that are used as tiebreak mechanisms in several team sports such as football and hockey as a mechanism design problem with order independence of the outcome in mind: which team starts kicking first should not matter for the outcome of the shootout but lead to even chances of winning when all players are equally talented.

Economists have long recognized that the effect of the order of actions in sequential contests on performance of the contestants is far from negligible. Examples in specific sequential individual and team contests are plenty, e.g., R\&D races (Fudenberg et al., 1983 and Harris and Vickers, 1985, 1987), dynamic games in general (Cabral, 2002), job promotions (Rosen, 1986), political campaigns (Klumpp and Polborn, 2006), music competitions (Ginsburgh and van Ours, 2003) as well as penalty shootouts in football matches (Apesteguia and Palacios-Huerta, 2010) and tennis matches (Cohen-Zada, Krumer, and Shapir, 2018 and the references therein). ${ }^{1}$ Clearly, an order of actions that provides a systematic first- or second-mover advantage to one of the parties may decrease the probability of the 'better' contestant to win, causing efficiency and fairness issues. Therefore, a focal direction is to aim for order independence in such team contests.

The history and experience of football and other sports' tiebreak mechanisms, known as penalty shootouts, present us a unique natural experiment to understand the strategic role of move order. Penalty shootouts currently constitute the only way to determine the winning team when the score is tied in major football elimination tournament matches after the regular 90-minute period and the 30-minute extra time, known as overtime. It is customary to use tiebreak mechanisms in many other sports as well to determine the eventual winner when the regular match ends with a tie, e.g., tennis, ice hockey, field hockey, water polo, handball, cricket, and rugby.

In a football shootout, since 1970 each team takes five penalty kicks from the penalty mark in fixed order $(A B A B$ for short, meaning that Team A kicks first then Team B kicks, then in the second round Team A kicks first again and so on), and the order of the kicks has always been decided by the referee's initial even coin toss. If the shootout score is tied after each team takes five penalty kicks, sudden-death rounds are reached, which go on until the tie is broken, such that the kicking order remains the same as regular rounds. ${ }^{2}$

A particular observation shared by multiple empirical studies regarding football penalty shootouts is that the degree of how much the kicking order in the ABAB mechanism matters may differ across different football competitions/traditions. For example, although kicking order does not matter for the German national cup, the Spanish national cup shootouts favor first-kicking teams significantly. On the other hand, in English cups, the first-kicking team has only a slight advantage. $^{3}$ Hence, in different tournament/country environments, ABAB leads to different fo-

\footnotetext{
${ }^{1}$ Football is called soccer in some countries, most notably in the US and Australia.

${ }^{2}$ Recently some top-level competitions started to experiment with alternating-order shootout mechanism $A B B A$, where order of kicking alternates after each round. See Appendix D.2 more on background on ABBA.

${ }^{3}$ See Table 5.1 in Palacios-Huerta (2014) and Table 1 in Kocher, Lenz, and Sutter (2012), which are summarized in Figure A.1 in Appendix D.1.
} 
cal outcomes in terms of first- and second-moving teams' winning chances. In addition, some researchers provide evidence that the first-kicking team wins significantly more often overall with ABAB, while some others dispute some of that evidence. ${ }^{4}$ No study, however, provides evidence that the second-kicking team wins more often overall.

Shootouts tend to be shorter and more structured than a regular match. They can be modeled like dynamic versions of contests. We introduce such a model in which the kickers not only care about their team's winning the shootout but also about the individual performance they display during taking their penalty shot. We provide empirical evidence to support this modeling assumption from Bar-Eli and Azar (2009) and Almeida, Volossovitch, and Duarte (2016): even high level players often aim at safer spots where the kick can be saved more often by the goalie than optimal spots, which provide higher chances of scoring, but also higher chances of kicking out. To capture this feature of penalty kicks, we assume that for a kicker, a save of his kick by a goalie is less irritating and more desirable than kicking the penalty out (as in the former case, the miss is caused by somebody else's, i.e., goalkeeper's, luck or effort, but not by the kicker's own mistake as in the latter case). We explain this empirical evidence in detail in Section 2.

Then we define order independence as the requirement that equally balanced teams - in terms of their players' shootout abilities - have equal chance of winning any time when the score is tied at the beginning of any round, i.e., after equal numbers of attempts, under all state-symmetric equilibria of the induced shootout game. ${ }^{5,6}$ Note that this property has implications only when the score is tied at the beginning of a round but is silent when it is not tied. Thus, it implies ex-ante fairness, i.e., an equal chance of winning at the beginning of the shootout at all state-symmetric equilibria even following a totally unfair coin toss.

First, we characterize order-independent mechanisms in regular rounds in Theorem 1. All exogenous mechanisms that have a predetermined kicking-order pattern - with one exception - are found to be order dependent, even if the sudden-death rounds were order independent for these mechanisms, e.g., even if the winner were determined by an even coin flip in sudden death. There is only one class of exogenous order-independent mechanisms, in which the kicking order after Round 1 is determined by an even coin flip in each round.

An important implication of this finding is that, as $\mathrm{ABAB}$ and $\mathrm{ABBA}$ have exogenous orders, regardless of the initial coin flip to determine which team goes first, they are both order dependent in regular rounds.

The whole class of order-independent mechanisms in regular rounds has the feature that when

\footnotetext{
${ }^{4}$ See Apesteguia and Palacios-Huerta (2010) for evidence on the first kicking team winning significantly more often. Kocher, Lenz, and Sutter (2012), on the other hand, dispute this finding. Later Palacios-Huerta (2014) uses a larger data set to find again a first-mover advantage (see also Figure A.1 in Appendix D.1).

${ }^{5} \mathrm{~A}$ precursor of our concept of order independence can be found in Che and Hendershott (2008), who use it for only one round in which teams take turns.

${ }^{6} \mathrm{~A}$ state-symmetric equilibrium is a Markov perfect Bayesian equilibrium in which each kicker uses the same strategy when the state of the game defined by the score difference and kicking order is (symmetrically for each team) the same at the round he moves.
} 
the score is tied at the beginning of a round, the kicking order does not matter for that round. Thus, we obtain order independence at the beginning of the shootouts.

On the other hand, all other order-independent mechanisms have an endogenous kicking-order determination when score is not tied at the beginning of a round: the probability of which team moves first in this round is the same for both teams whenever they are in each other's shoes. E.g., consider two cases in which Team 1 is ahead $1-0$ and Team 2 is ahead $0-1$ at the beginning of Round 2, respectively; then Team 1's probability of moving first in Round 2 in the first case is the same as Team 2's probability of moving first in the second case. We refer to the class of mechanisms that fully characterize the continuum of order-independent mechanisms in regular rounds as uneven score symmetric.

Then we move from regular rounds to sudden-death rounds. That is, we consider order independence in sudden-death rounds when the score is tied after regular rounds. Interestingly, as the score is never uneven at the beginning of any sudden-death round, both ABAB and ABBA are tautologically uneven score symmetric in sudden-death rounds. ABAB induces an infinite game such that each sudden-death round is a repetition of the previous one and the game only ends when one team scores and the other does not. We show that ABAB is not order-independent in sudden-death rounds for reasons very different than those for regular rounds (Theorem 2). It turns out that $\mathrm{ABAB}$ leads to multiplicity of equilibria as in that game: For every equilibrium in which Team 1 wins more often, there is a dual equilibrium in which Team 1 and Team 2 players swap their strategies, and hence Team 2 wins more often, and yet there is always an equilibrium in which both teams win with equal probability. ${ }^{7}$

On the other hand, we show in Theorem 3 that alternating order of the teams as in ABBA is enough to rule out asymmetric equilibria in which teams win with different probabilities as state-symmetric and to sustain order independence back. Then we provide a large class of orderindependent mechanisms in sudden-death rounds in Theorem 4.

Order independence implies ex-ante fairness. If there is a unique equilibrium of ABAB after who goes first is determined and this dictates the first moving team wins at least as much probability as the second moving team (or just the opposite), then ABAB will be ex-ante fair as long as it determines who kicks first with an even coin toss. However, an interesting implication of our result is that despite the initial even coin toss, $\mathrm{ABAB}$ is not ex-ante fair if it has multiple equilibria in sudden-death rounds. Under the sufficient conditions for equilibrium multiplicity we pin down in Theorem 6 in Appendix B, it has a state-symmetric equilibrium in which one team wins with a higher probability regardless it kicks first or second (such an equilibrium also exists for the other team winning more often). This implies ABAB is not ex-ante fair as we cannot tell, in general, which state-symmetric equilibrium will be selected.

As our notion of order independence involves all state-symmetric equilibrium winning chances

\footnotetext{
${ }^{7}$ We also show in Appendix $\mathrm{H}$ that it is possible to devise an equilibrium refinement using the sequential kicking aspect in a single round so that any potential equilibrium candidate with the second-mover advantage does not survive this refinement.
} 
being equal, this is different from the commonly used notion of procedural ex-ante fairness (or also commonly referred to as symmetry). ${ }^{8}$ We also give a tight characterization of practical procedurally fair and order-independent mechanisms that can be adopted in real-life shootouts in Theorem 5 depending on the difficulty level of the shootouts. ${ }^{9}$ We can classify penalty shootouts as easy task or difficult task based on the goal scoring probability. A shootout is easy if the goal probability is greater than $50 \%$ for any kick in any round and it is difficult if the goal probability is less than $50 \%$. A football shootout is an example of an easy task, while a hockey shootout is an example of a difficult task. One particular class of mechanisms in easy-task shootouts is the behind-first mechanisms: the team which is behind at the end of a round goes first in the next round; but if the score is tied after any round, then any random or fixed exogenous or endogenous order is admissible at the next round. We conclude by elaborating which of these mechanisms are practical and procedurally fair as well as order independent. ${ }^{10}$

\section{Background: Football Shootouts}

Until 1970, elimination matches in football that were tied after extra time were either decided by a coin toss or replayed in two days if it was a finals match. Finally, the events in the 1968 European Football Championship that involved coin toss as tie breaks led FIFA in 1970 to try penalty shootouts instead. Given that the current shootout mechanism, ABAB, is no panacea, FIFA, England, and The Netherlands are now experimenting with alternating the orders of kicking teams, i.e., using the alternating-order ABBA scheme.

The outcomes of players' kicks pertain to their teams as well as to themselves. From the team's perspective a goal is preferred to a non-goal, and clearly there is no difference at all between a saved kick and a kick that misses the goal.

\footnotetext{
${ }^{8}$ Flipping a fair coin to determine which team will kick first makes $\mathrm{ABAB}$ only procedurally fair under such equilibrium multiplicity. However, as we have explained above, who goes first has no importance whatsoever for an order-independent mechanism in Round 1. Only when the score is not tied at the beginning of a round, an order-independent mechanism needs to endogenously regulate the kicking order, which is not done by $\mathrm{ABAB}$ even though it starts with an initial even coin toss.

${ }^{9}$ See Appendix F for further details of this characterization.

${ }^{10}$ Economists have long focused on design of sports contests, such as the optimal number of entrants/teams in a race/league, the optimal structure of prizes (revenue sharing) for a tournament (league), and so on (see Szymanski, 2003 for a review of this particular literature and see Bergantinos and Moreno-Ternero, 2018 for a more recent paper). Two other papers in this strand are about sports competition restructuring through efficient matching (Frechétte, Roth, and Ünver, 2007) and fair matching of teams in a competition (Boczoń and Wilson, 2018). After initial draft of our paper became publicly available (Anbarc1, Sun, and Ünver, 2015), order of moves in sports competitions is also studied by Echenique (2017) and Brams, Ismail, Kilgour, and Stromquist (2018). The first study shows under certain exogenous scoring probability assumptions ABBA is fairer than ABAB. The latter study uses the idea of the catch-up rule (Brams and Ismail, 2018) to introduce schemes to increase competitiveness in various sports, where parties take turns in service sports such as tennis and volleyball. Server is the loser of the previous point in catch-up rule. Csató (2020) introduces a variant of this to make it even fairer.
} 
Bar-Eli and Azar along with Lurie have written key papers analyzing and modeling the behavior of penalty kickers (Bar-Eli and Azar, 2009; Bar-Eli, Azar, and Lurie, 2009). Based on the distributional data of 311 penalty kicks from important top league games, World Cup, and other international championships that had televised or archived footage in Israel's major TV networks, Bar-Eli and Azar (2009) statistically find that very frequently kickers use a safer strategy of kicking the ball to the lower part of the goal, and this is stopped frequently by the goalkeepers. On the other hand, they use the risky strategy of kicking the ball to the upper part of the goal less often, although it results with $100 \%$ success rate when it hits the target. They conclude based on the data that it is optimal to kick this portion of the goal rather than the frequented lower portion. They explain the reason as follows for our purposes (on page 189):

"A third possible reason for kicking low might be that players prefer to take the risk that the goalkeeper will stop the ball rather than the risk that their kick will miss the goal, because in the former case it will not be perceived as being entirely their fault that a goal is not scored, while in the latter case it will be. Such preferences can lead to wrong decisions by players and their team will be hurt."

Almeida, Volossovitch and Duarte (2016) confirm these findings using a similar analysis of the penalty kicks in UEFA competitions between 2010 and 2015. They use a data set consisting of a total of 536 penalties. We give some of the summary statistics of their regression analysis here. They show that when the shots are aimed low, they are more likely saved (out of 320 kicks, 62 saved - 19.3\%, 247 goals - 77\%, 11 missed out - 3.7\%, for left-lower and left-right) compared to when they are aimed high (out of 80 kicks, 3 saved - 3.75\%, 69 goals $-86 \%$, and 8 missed out $10 \%$ for left-upper and right-upper). Observe that despite the fact that the successful strategy is kicking upper-left or upper-right of the goal based on data, only about $15 \%$ of the kicks were aimed in this region, while $60 \%$ of the kicks were aimed lower left or right. ${ }^{11}$ These pieces of evidence from multiple studies indicate that indeed kickers care about other outcomes besides scoring a goal. One rational explanation for their consistent behavior is they get higher disutility from missing the target altogether than their kick being saved by the goalkeeper as pointed out by Bar-Eli and Azar (2009). ${ }^{12}$ Our one dimensional goal model setup that we introduce in the next section is a proxy

\footnotetext{
${ }^{11}$ The rest were aimed lower or upper middle regions, with much lower success rate for lower-middle region of the goal.

${ }^{12} \mathrm{~A}$ famous anecdote supporting this explanation, the empirical evidence, and also our utility representation comes from Italy's Roberto Baggio, who had a stellar career and his five goals in the tournament helped Italy to reach the final's match of the 1994 World Cup against Brazil. With the shootout score at 3-2, as the last kicker in regular rounds Baggio had to score to keep Italy's chances alive. He aimed for the middle but the ball sailed over the crossbar. The following quote is from Baggio's (2001) autobiography.
}

"As for the penalty, I don't want to brag but I've only ever missed a couple of penalties in my career. And they were because the goalkeeper saved them not because I shot wide. That's just so you understand that there is no easy explanation for what happened at Pasadena. When I went up to the spot I was pretty lucid, as much as one can be in that kind of situation. I knew [the Brazilian goalie] Taffarel always dived so I decided to shoot for the middle, about halfway up, so he couldn't get it with his feet. It was an intelligent 
for the two dimensional goal for tractability purposes to capture these nuances in revealed kicker utility functions.

When we analyze our kicker optimal strategy, the resulting behavior mimics the empirical findings using our utility function representation: kickers end up aiming at a safer spot instead of goal-optimal spot so that they can avoid the higher likelihood outcome of kicking out. Therefore, our utility function provides a rational explanation for this revealed kicker behavior. ${ }^{13}$

Besides this compelling evidence regarding penalty kick performance of kickers relying how a goal is missed, the relevant literature also points out that overall players care about their own performance, besides their team's outcome in other dynamic team contests. Chapsal and Vilain (2019) provide evidence from international team squash tournaments that players care not only about their team's win or loss, but also their individual performance.

\section{Model}

\subsection{The Setup}

Two football teams, which we refer to as Team 1 ( $T_{1}$ in mathematical notation) and Team $2\left(T_{2}\right.$ in mathematical notation), are facing off in a penalty shootout. Each team shall take $n$ sequential rounds of penalty shots. Each round consists of one team kicking first, and, after observing the outcome of that shot, the second team taking the next shot. If one team scores more goals than the other at the end of $n$ rounds, then it wins the match. We refer to these $n$ rounds as the regular rounds. Throughout the paper we will assume that $n=2$. This is sufficient to characterize order independence and analyze the current scheme, ABAB, as well as other proposed mechanisms, such as the alternating-order mechanism, ABBA. Thus, with $n=2$, the analysis is tractable and yet rich enough to capture the multi-round feature of penalty shootouts. ${ }^{14}$

decision because Taffarel did go to his left, and he would never have got to the shot I planned. Unfortunately, and I don't know how, the ball went up three meters and flew over the crossbar. I failed that time. Period. And it affected me for years. It is the worst moment of my career. I still dream about it. If I could erase a moment from my career, it would be that one."

${ }^{13}$ We also infer from Baggio's quote in Footnote 12 that goalies typically feel the need to dive at the time the ball is kicked. This is because, at the optimal speed-accuracy combinations of world-class kickers, the kicked ball typically takes around 0.3 seconds to reach the goal line (see, e.g., Harford, 2006, Chiappori et al., 2002, and Palacios-Huerta, 2003), which is less than the total of (1) roughly 0.2 seconds' reaction time of the goalie to clearly recognize the kick direction of the ball first, plus (2) the time during his dive to reach the expected arrival spot of the ball before it reaches the goal plane. Hence, a goalie cannot afford to wait until he clearly observes the kick direction: to prevent a goal with non-trivial probability, he must commit to pick a side to dive - or alternatively to stay in the middle. As Baggio's quote also indicates, a shot aimed at the middle may be missed outright or may hit the feet or the legs of the diving goalie that cover part of the middle; thus, the shot can be saved even if the goalie dives.

${ }^{14}$ We have $n=3$ results in Appendix G, and no extra insight are obtained in this analysis. Similarly, we skip $n>3$ as the analysis becomes extremely cumbersome and lengthy. Although, we do not have a proof for $n>3$, we have no reason to suspect it would not generalize to this setting. 
If the shootout score is tied at the end of $n$ regular rounds, the format reverts to sudden death; that is, teams take an additional round of shots, and then, if one team scores while the other one does not, the former team wins the match; otherwise a further round of sudden-death penalty shots is taken. We refer to the sudden-death rounds as $n+1, n+2, \ldots$.

Since potentially the match can continue forever, we assume that each team consists of an infinite number of kickers and that each kicker takes at most one shot. ${ }^{15}$

A penalty kick consists of a probabilistic event with three outcomes: Either a goal is scored $(\mathrm{G})$, the shot goes out $(\mathrm{O})$, or the shot is saved by the goalie $(\mathrm{S})$. The latter two outcomes lead to the same score for the team: a goal is not scored.

While each kicker is a strategic player, for tractability the goalie is modeled as a probabilistic machine. The goalie waits in the middle of the goal line prior to the shot. He jumps to one side or the other with probabilities $\frac{1}{2}: \frac{1}{2}$ prior to the penalty shot, as he needs to react early to have any realistic chance to save the kick (see Footnote 13). So with probability $\frac{1}{2}$ he reaches to the same side of the goal as the kick. Hence, we model the goal line as a one-dimensional line segment $[0,1]$, where $x=0$ refers to the center of the goal, and $x=1$ refers to the goal pole on the side of the kick.

Each kicker, who is a single-round player in our game, has an action summarized as aiming at coordinate $x \in[0,1]$ of the goal line, which we refer to as the intended spot. When a kicker aims at $x$, the exact spot the ball reaches on the goal line is determined by a continuous probability density function $\sigma_{x}$ in a closed support $\left[\underline{\epsilon}_{x}, \bar{\epsilon}_{x}\right]$ for some $\bar{\epsilon}_{x}>x>\underline{\epsilon}_{x}$. The spot the ball reaches, $\chi$, is observable by all other players, but not the intended spot, $x$. Both $x$ and $\chi$ are observable by the kicker himself. Moreover, given that the shot is aimed at $x$, there is a $P_{G}(x)$ probability that a goal will be scored and a $P_{O}(x)$ probability that the shot will go out. Hence, the shot is saved by the goalie with probability $1-P_{G}(x)-P_{O}(x) .{ }^{16}$ We assume that $P_{G}, P_{O}$, and $\sigma_{x}$ for all $x \in[0,1]$ are all common knowledge. The probability of kicking out and the probability of scoring are all reduced form from the model in which there are two sides of the goal and the kicker also decides which side to kick.

We assume that $P_{G}$ is a twice continuously differentiable strictly concave function, which reaches its maximum at some interior $\bar{x} \in(0,1) \cdot{ }^{17}$

\footnotetext{
${ }^{15}$ In reality, each football player can take at most one shot, unless all players in his team have already kicked penalty shots. As each team consists of 11 players, 11 shots need to be taken by each team before any player can kick a second shot. As $n=5$, this happens very rarely.

${ }^{16}$ Actually $P_{G}$ and $P_{O}$ are summary functions obtained from the following process: As mentioned before, the spot the ball reaches, $\chi$, is observable by all other players, but not the intended spot, $x$. If $\chi>1$, then the ball goes out. So $P_{O}(x)=\int_{y=1}^{\bar{\epsilon}_{x}} \sigma_{x}(\chi) d \chi$. On the other hand, the goalkeeper can save the ball that arrives at spot $\chi$ with probability $S(\chi)$, which is a continuous function. Thus, $P_{G}(x)=\int_{\underline{\epsilon}_{x}}^{1}[1-S(\chi)] \sigma_{x}(\chi) d \chi$. Hence, we assume that the family of densities $\left\{\sigma_{x}\right\}_{x \in[0,1]}$ and save probability function $S$ have all the properties that need the below restrictions to hold for $P_{G}$ and $P_{O}$.

${ }^{17}$ In fact $P_{G}$ is not concave around 0 , and it is decreasing, as the ball can go both sides of the middle, $x=0$, when it is aimed at $x=0$. Nevertheless, we assume the goal-maximizing point is farther to the right. Therefore, without
} 
Although so far we developed our theory taking football as our primary application, the insights we discover apply to other contests and sports. In particular, we can classify penalty shootouts as easy-task or difficult-task based on the goal scoring probability $P_{G}(x)$. A shootout is easy if $P_{G}(x)>\frac{1}{2}$ for all $x \in[0, \bar{x}]$. A shootout is difficult if $P_{G}(x)<\frac{1}{2}$ for all $x \in[0, \bar{x}]$. A football shootout is an example of an easy task, while a hockey shootout is an example of a difficult task. 18 This distinction will not matter in our results until we discuss different efficiency notions and practical design considerations in Appendix F. We assume that the shootout is either easy or difficult, but not mixed, throughout the paper. Thus, our analysis will focus on these two cases throughout.

Function $P_{O}$, on the other hand, is an increasing twice continuously differentiable convex function. Increasing $P_{O}$ is straightforward to motivate: the closer to the middle the ball is aimed, the lower is the chance that the ball will go out. Single-peakedness of $P_{G}$ is also easy to motivate: Whenever the ball is aimed at low $x$ values, it can be saved with a higher chance by the diving goalie (see Footnote 18 for hockey dynamics). For higher $x$ values, although the goalie's chances of saving the ball decrease as he may no longer be able to reach it, the chances of the ball going out increase. Hence, it is easy to motivate the unique spot $\bar{x}$, which maximizes the goal probability. We will refer to it as the goal-optimal spot. Concavity of $P_{G}$ and convexity of $P_{O}$ are primarily assumed for the tractability of our analysis, and do not play any other major role for the interpretation of our results.

We assume that each kicker on both teams is identical in ability and has the same goal-scoring and kicking-out probability. ${ }^{19}$

\subsection{Shootout Mechanisms and the Shootout Game}

A shootout mechanism is a function, $\phi$, that assigns a probability $\phi\left(h^{k-1} ; g_{T_{1}}: g_{T_{2}}\right)$ to Team 1 kicking first in Round $k$, given the sequence of first-kicking teams in the first $k-1$ rounds is $h^{k-1}=\left(h_{r}^{k-1}\right)_{r=1}^{k-1}$ where $h_{r}^{k-1} \in\left\{T_{1}, T_{2}\right\}$ is the team that kicked first in Round $r$ and $g_{T_{1}}: g_{T_{2}}$ is the score (i.e., the goals scored by Team 1 and Team 2 , respectively) at the beginning of Round $k$. Thus, the probability of Team 2 kicking first in Round $k$ is $1-\phi\left(h^{k-1} ; g_{T_{1}}: g_{T_{2}}\right)$.

Each shootout mechanism $\phi$ induces a hidden-action extensive-form game, which we will simply refer to as the game, such that the exact spot that each kicker aims the ball on the goal line is unobservable by others. Given $\left(h^{k-1} ; g_{T_{1}}: g_{T_{2}}\right)$, for Rounds $k=1,2, \ldots$, i.e., the order of first-

loss of generality, we use a strictly concave $P_{G}$.

${ }^{18}$ As alluded to in the Introduction, scoring a penalty shot is easy in football with a success probability greater than 59\% in each round (for example, see Apesteguia and Palacios-Huerta, 2010), while it is difficult in hockey with a probability less than 35\% (for example, see http://businessofhockey.wordpress.com/2015/01/ 04/a-deep-look-into-advanced-shootout-statistics/ retrieved on Feb 13, 2018). As different from football, usually hockey goalies do not dive one side of the goal as the goal is substantially smaller, hence, the goal probabilities are uniformly less than $50 \%$.

${ }^{19}$ We consider one particular exception in Appendix E, where we assess chances of unbalanced teams. 
kicking teams in the previous $k-1$ rounds $h^{k-1}$, and feasible scores $g_{T_{1}}: g_{T_{2}}$, the Nature determines with probability $\phi\left(h^{k-1} ; g_{T_{1}}: g_{T_{2}}\right)$ Team 1 kicking next first and probability $1-\phi\left(h^{k-1} ; g_{T_{1}}: g_{T_{2}}\right)$ Team 2 kicking next first. Then a kicker of the first-kicking team takes the penalty shot, observing the state and the history of the outcomes of all the shots up to that point as goal, out, or save. The kicker aims at his intended spot $x \in[0,1]$ to maximize his expected individual payoff (which we explain in the next paragraph). Then the Nature determines with probability distribution $P_{G}(x), P_{O}(x), 1-P_{G}(x)-P_{O}(x)$ whether the penalty kick results in a goal, goes out, or is saved, respectively. After the outcome of this shot is observed, the other team's kicker takes a penalty shot, observing the history of the outcomes of the shots up to that point. We continue until the end of regular rounds, Round $k=n$, similarly. If the score is tied after the last regular round, sudden-death rounds take place until the tie is broken at the end of a sudden-death Round $k>n$.

Each kicker aims to maximize his expected individual payoff in the game. Each kicker's payoff function consists of two additive components. The first is the utility received when his team wins or loses the shootout: $V_{W}$ is the team win payoff and $V_{L}<V_{W}$ is the team loss payoff. This component of the payoff is common to each kicker on the team. The second component of the individual payoff consists of an individual outcome based valuation: If the kicker scores a goal, he gets payoff $U_{G}>0$; if he kicks the ball out, he receives payoff $U_{O}<0$; if the goalie saves the kick, he receives payoff $U_{S}=0$. The latter is a normalization which guarantees that scoring a goal is the most desirable outcome for the kicker, and kicking the ball out is less desirable than kicking the ball inside the goal frame and yet the goalie saves it. With this normalization, we can also drop a variable from our notation without affecting our analyses. Thus, the overall ex-post payoff of a kicker $i$ of Team $k$ is then

$$
u_{i, T_{k}}=V_{t}+U_{p}
$$

where $t \in\{W, L\}$ refers to the overall team outcome, win or loss, and $p \in\{G, O, S\}$ refers to the kicker's penalty outcome, goal, out, or save.

An information set is $H \in \mathcal{H}_{i, T_{k}}$, which is the collection of information sets that kicker $i \in\{1,2, \ldots\}$ of Team $k \in\{1,2\}$ can move, consists of the exact spot the ball went to for each of the previous kicks, the team of each kick, and whether the kick was scored as a goal, went out, or was saved by the goalie. Thus, they are all observable by kicker $i$ of Team $k$ moving in information set $H$. What is not observable by him is the intended spots of previous kicks. Each information set also has an associated round (without loss of generality indexed with the kicker, i.e. $i$ 'th round), order of kicking in the round as $1^{\text {st }}$ or $2^{\text {nd }}$, and a current score difference between Team 1 and Team 2. We refer to all of this observable information as the state of the information set. Note that from the point of view of the kicker, who is a single-shot player in the game, all payoff-relevant information of an information set is given through its state.

A pure strategy $X_{i, T_{k}}: \mathcal{H}_{i, T_{k}} \rightarrow[0,1]$ is a function from the collection of information sets that Team $k$ 's $i$ 'th kicker's move to the intended spots that he can target while taking the penalty shot.

As alluded to before, this is a hidden-action sequential game, as what each player observes 
about others' kicks are only where the ball goes and whether the kick was a goal, out, or a save in previous kicks, but not the intended spot towards which the ball was kicked. Hence, as a kicker takes a penalty shot, he has a belief over intended spots of previous kicks. Formally, a belief $\mu(H)$ is a function that maps each information set $H \in \mathcal{H}_{i, T_{k}}$ that Team $k$ 's $i$ 'th kicker's move with positive probability to a probability distribution over histories of actions taken that would lead to the same information set.

\subsection{Markov Perfection and State-Symmetric Equilibria}

Our solution concept is state-symmetric (perfect Bayesian) equilibrium, in which strategies in regular rounds depend only on the state of the game, i.e., on the round number, kicking order, and score difference; strategies in sudden-death rounds depend only on the current kicking order and score difference. The strategies in state-symmetric equilibria are memoryless in that they depend only on the current state.

A perfect Bayesian equilibrium in the game of shootout mechanism $\phi$ is an assessment, i.e., a strategy profile and a belief profile pair $\left[X=\left(X_{i, T_{k^{\prime}}}\right)_{i \in\{1,2, \ldots\}, k^{\prime} \in\{1,2\}}, \mu=\right.$ $\left.(\mu(H))_{H \in \mathcal{H}_{i, T}, i \in\{1,2, \ldots\}, k^{\prime} \in\{1,2\}}\right]$ such that for any $k, \ell \in\{1,2\}$ s.t. $k \neq \ell, i \in\{1,2, \ldots\}$, and $H \in \mathcal{H}_{i, T_{k}}$,

- spot $X_{i, T_{k}}(H) \in[0,1]$ maximizes the expected value of all possible payoffs $u_{i, T_{k}}$ at information set $H$, given $X_{-i, T_{k}}$ and $X_{T_{\ell}}=\left(X_{j, T_{\ell}}\right)_{j \in\{1,2, \ldots\}}$ among all spots in $[0,1]$; and

- belief $\mu(H)$ is consistently derived by Bayes' rule from $\phi, X, P_{G}, P_{O}, \mu\left(H^{\prime}\right)$ for all $H^{\prime} \neq H$.

Each kicker is a one-shot player and maximizes his individual expected payoff over his ex-post payoffs $u_{i, T_{k}}$ defined in Equation 1. The exact formulation of this expected payoff will be made clear later in our analysis.

In this game, once the equilibrium strategies are found, beliefs are straightforward to construct. At any information set $H$, the kicking player believes with probability one that other kickers before him used equilibrium strategies. This is because the payoffs explicitly depend on the actual outcome of each kick, which is observable as Goal (G) or No Goal (NG), but not on the intended spots of kicks (which are not observable). Further, beliefs will not play a role in finding the optimal strategies in equilibria as the kicker decides on his best action by taking into consideration only future players' kicks, not those of the past ones. We will not explicitly calculate the beliefs from this point on, except when we refine the possible multiple equilibria of ABAB in Appendix $H$.

We will focus on a Markovian symmetric equilibrium concept (i.e., unless we refine the possible multiple equilibria of $\mathrm{ABAB})$ :

A state-symmetric assessment $(X, \mu)$ is defined as

- In regular rounds: $X_{i, T_{k}}(H)=X_{i, T_{\ell}}\left(H^{\prime}\right)$ and $\mu(H)=\mu\left(H^{\prime}\right)$ for any $k, \ell \in\{1,2\}$ where both information sets $H \in \mathcal{H}_{i, T_{k}}$ and $H^{\prime} \in \mathcal{H}_{i, T_{\ell}}$ pertain to the same Regular Round $i \leq n$, 
and the same kicking order, $1^{\text {st }}$ or $2^{\text {nd }}$, in the round while the score difference between Team 1 and Team 2 in $H, s$, and in $H^{\prime}, s^{\prime}$, satisfy $s=-s^{\prime}$ if $\ell \neq k$ and $s=s^{\prime}$ if $\ell=k$.

- In sudden-death rounds: $X_{i, T_{k}}(H)=X_{j, T_{\ell}}\left(H^{\prime}\right)$ and $\mu(H)=\mu\left(H^{\prime}\right)$ for any $k, \ell \in\{1,2\}$ where information sets $H \in \mathcal{H}_{i, T_{k}}$ and $H^{\prime} \in \mathcal{H}_{j, T_{\ell}}$ involve (possibly different) Sudden-death Rounds $i, j>n$ but they refer to the same kicking order, $1^{\text {st }}$ or $2^{\text {nd }}$, while the score difference between Team 1 and Team 2 in $H, s$, and in $H^{\prime}, s^{\prime}$, satisfy $s=-s^{\prime}$ if $k \neq \ell$ and $s=s^{\prime}$ if $k=\ell$.

A state-symmetric assessment in sudden-death rounds, for instance, dictates that two players on the same team or different teams will exactly aim at the same intended spot and have exactly the same beliefs if they were in each other's shoes. Note that before every sudden-death round the score is identical if the game reaches it, while before each regular round after Round 1 it could be different. Unlike the sudden-death rounds, the number of regular rounds is finite, and therefore the round number as well as the kicking order and score would matter in regular rounds. Therefore, even if two teams are tied in different regular rounds, the players who kick first need not use the same strategy in those two rounds.

A state-symmetric equilibrium of a shootout mechanism $\phi$ is defined as a state-symmetric perfect Bayesian equilibrium of the game induced by $\phi$. This solution concept is identical to symmetric Markov-perfect equilibrium if one were to ignore the beliefs and focused only on strategies assuming that each state of the game spans a subgame of the game. As noted above, beliefs play no role other than equilibrium selection when there are multiple equilibria; this is without loss of generality.

\subsection{Order Independence}

Using the concept of state-symmetric equilibrium, we now define the key design concept in our analysis as follows: an assessment $(X, \mu)$ of the game induced by mechanism $\phi$ is order independent if for all problems with equally balanced teams (i.e., for any underlying utility values $V_{W}, V_{L}, U_{G}, U_{O}$, and goal and ball going out probability functions $\left.P_{G}, P_{O}\right)$, at any $\left(h^{k-1} ; g_{T_{1}}: g_{T_{2}}\right)$ with $g_{T_{1}}=g_{T_{2}}$ (i.e., when they are tied at the beginning of Round $k$ for any $k$ ), each team has exactly a $50 \%$ chance of winning. In order to ensure order independence, we will focus on shootout mechanisms whose all state-symmetric equilibria are order independent. We will refer to such mechanisms as orderindependent mechanisms. Note that it is not the shootout mechanism that is procedurally fair, but its state-symmetric equilibria that need to be order independent.

We will analyze order independence in sudden-death rounds as well. It will be useful to formally define this concept. A mechanism is order independent in sudden-death rounds if, for all problems with equally balanced teams, for any Sudden-death Round $k>n$, at any $\left(h^{k-1} ; g_{T_{1}}: g_{T_{2}}\right)$ with $g_{T_{1}}=g_{T_{2}}$, (i.e., when they are tied at the beginning of Round $k$ ), each team has exactly a $50 \%$ chance of winning. 
We will determine whether ABAB's equilibria are order independent and inspect other plausible mechanisms by characterizing the class of order-independent mechanisms in regular rounds and providing a large class of order-independent mechanisms in sudden-death rounds.

\section{Analysis: A Kicker's Optimization Problem}

We first analyze each kicker's optimization problem for a given mechanism $\phi$ and other agents' strategies. The best response determination problem of the $i$ 'th kicker of Team $k$, denoted by $\kappa \equiv\left(i, T_{k}\right)$, boils down to

$$
\max _{x_{\kappa} \in[0,1]} U_{\kappa}\left(x_{\kappa} ; W_{G, \kappa}, W_{N G, \kappa}\right) \equiv\left(P_{G}\left(x_{\kappa}\right) W_{G, \kappa}+\left[1-P_{G}\left(x_{\kappa}\right)\right] W_{N G, \kappa}\right)+\left(P_{G}\left(x_{\kappa}\right) U_{G}+P_{O}\left(x_{\kappa}\right) U_{O}\right)
$$

where $P_{G}\left(x_{\kappa}\right) U_{G}+P_{O}\left(x_{\kappa}\right) U_{O}$ is Kicker $\kappa$ 's expected individual kick payoff, and $P_{G}\left(x_{\kappa}\right) W_{G, \kappa}+[1-$ $\left.P_{G}\left(x_{\kappa}\right)\right] W_{N G, \kappa}$ is Kicker $\kappa$ 's expected continuation team payoff given expected continuation values $W_{G, \kappa}$ conditional on he scores and $W_{N G, \kappa}$ conditional on he does not score. These values, $W_{G, \kappa}$ and $W_{N G, \kappa}$, are functions of the shootout mechanism, the score difference, the round number ( $i$ in this case), kicking order in that round, and other players' strategies. We drop them from our notation for simplicity.

Hence, the necessary first-order conditions for an interior maximum turn out to be

$$
P_{G}^{\prime}\left(x_{\kappa}^{*}\right)\left(W_{G, \kappa}-W_{N G, \kappa}+U_{G}\right)+P_{O}^{\prime}\left(x_{\kappa}^{*}\right) U_{O}=0 .
$$

The second-order conditions lead to the first-order conditions being sufficient, since we have

$$
P_{G}^{\prime \prime}\left(x_{\kappa}^{*}\right)\left(W_{G, \kappa}-W_{N G, \kappa}+U_{G}\right)+P_{O}^{\prime \prime}\left(x_{\kappa}^{*}\right) U_{O}<0,
$$

which follows from the facts that $P_{G}^{\prime \prime}<0, W_{G, \kappa}-W_{N G, \kappa} \geq 0,{ }^{20} U_{G}>0, P_{O}^{\prime \prime} \geq 0$ and $U_{O}<0$. Hence, if an interior maximum exists, it is unique given $W_{G, \kappa}-W_{N G, \kappa}$. We will refer to $W_{G, \kappa}-W_{N G, \kappa}$ as the expected marginal contribution of the kicker to his team. We turn our attention to analyze the properties of the optimum for a kicker.

Proposition 1 At any interior best response of Kicker $\kappa, x_{\kappa}^{*}<\bar{x}$ is the kicker-optimal spot, and the higher his expected marginal contribution, the higher is his goal-scoring probability. ${ }^{21}$

Also note that if kicking out and the goal being saved were valued equally, i.e., $U_{O}=U_{S}=0$, then $x_{\kappa}^{*}=\bar{x}$, i.e., $x_{\kappa}^{*}$ would be also goal-optimal. But since $x_{\kappa}^{*}<\bar{x}$ because $U_{O}<U_{S}=0$, a kicker chooses to kick more conservatively. The relative magnitude $P_{G}^{\prime}\left(x_{\kappa}^{*}\right) / P_{O}^{\prime}\left(x_{\kappa}^{*}\right)$ as well as magnitudes

\footnotetext{
${ }^{20}$ Although $W_{G, \kappa}-W_{N G, \kappa}$ is endogenous and can potentially be negative, it is positive for all the mechanisms we consider.

${ }^{21}$ We provide Proofs of Proposition 1 and Theorem 1 in Appendix A and the proofs of other results in the main text are in Appendix C.
} 
of expected marginal contribution $W_{G, \kappa}-W_{N G, \kappa}, U_{O}$, and $U_{G}$ determine how much he shaves off the goal-optimal spot to determine his kicker-optimal spot. ${ }^{22}$

Observe that the choice of kicker-optimal spot is costly because of the probability of kicking the shot out, and therefore, it can be interpreted as a choice of an effort level. The goal-optimal spot also translates in the goal-optimal effort. The closer the spot gets (from the center) to the goaloptimal spot, it can be interpreted as exerting higher effort. From now on, when it is convenient, we will use this analogy more freely and refer to the choice of targeted spot as exerting an effort.

\section{$5 \quad$ Regular Rounds}

In this section we characterize order-independent mechanisms in regular rounds assuming that once the shootout extends to sudden-death rounds, there is an order-independent ending i.e., each team wins with the same probability. ${ }^{23} \mathrm{~A}$ natural question is whether ABAB or ABBA is order independent in regular rounds.

We define $\mathrm{ABAB}$ as follows: The first kicker is determined before Round 1 with an even lottery and then the procedure continues with the same kicking order throughout. Formally, (the fixedorder mechanism) $\mathrm{ABAB} \phi^{\mathrm{ABAB}}$ is defined as follows:

$$
\phi^{\mathrm{ABAB}}(\emptyset ; 0: 0)=0.5 \text { and } \phi^{\mathrm{ABAB}}\left(h^{k-1} ; g_{T_{1}}: g_{T_{2}}\right)= \begin{cases}1 & \text { if } h_{1}^{k-1}=T_{1} \\ 0 & \text { if } h_{1}^{k-1}=T_{2}\end{cases}
$$

for all rounds $k \geq 2$, orders of first-kicking teams in the previous $k-1$ rounds $h^{k-1}$, and feasible scores $g_{T_{1}}: g_{T_{2}}$ at the beginning of Round $k$.

On the other hand, formally (the alternating-order mechanism) ABBA $\phi^{\mathrm{ABBA}}$ is defined as follows:

$$
\phi^{\mathrm{ABBA}}(\emptyset ; 0: 0)=0.5 \text { and } \phi^{\mathrm{ABBA}}\left(h^{k-1} ; g_{T_{1}}: g_{T_{2}}\right)= \begin{cases}1 & \text { if } h_{k-1}^{k-1}=T_{2} \\ 0 & \text { if } h_{k-1}^{k-1}=T_{1}\end{cases}
$$

for all Rounds $k \geq 2$, orders of first-kicking teams in the previous $k-1$ rounds $h^{k-1}$, and feasible scores $g_{T_{1}}: g_{T_{2}}$ at the beginning of Round $k$.

It turns out that neither $\mathrm{ABAB}$ nor $\mathrm{ABBA}$ is order independent in regular rounds, even if they were order independent in sudden death. ${ }^{24}$ In fact a large class of intuitive mechanisms turns out to be order dependent. In addition, a large class of mechanisms, which we refer to as exogenous mechanisms, turns out to be order dependent except for one exception. A mechanism

\footnotetext{
${ }^{22}$ If the game involved only $n=1$ round and ties at the end were resolved through a coin toss, we would have $W_{G, \kappa}-W_{N G, \kappa}=\frac{V_{W}-V_{L}}{2}$.

${ }^{23}$ For example, an even coin flip can determine who wins if the score is tied after regular rounds, or any other order-independent mechanism in sudden-death rounds can be used.

${ }^{24}$ In Section 6.2 we show that ABAB is not order independent in sudden-death rounds, while ABBA is. Therefore, there are two sources of order dependence of $\mathrm{ABAB}$ while the order dependence of $\mathrm{ABBA}$ comes from regular rounds only.
} 
$\phi$ is exogenous if, for all rounds $k$, and kicking orders $h^{k-1}$ regarding the beginning of round $k$, $\phi\left(h^{k-1}: g_{T_{1}}: g_{T_{2}}\right)=\rho(k)$ for some function $\rho$, i.e., who goes first in each round is determined independent of the current score but as a function of the current round. Hence, both ABAB and ABBA are exogenous, and even the version of ABBA in which the 5th round's kicking order is randomly determined is exogenous.

One particularly interesting exogenous mechanism, however, is the random-order mechanism $\phi$, which determines who goes first in every round using an unbiased lottery at the beginning of that round, that is $\phi\left(h^{k-1} ; g_{T_{1}}, g_{T_{2}}\right)=\frac{1}{2}$ for all $k$. Despite its impracticality, this particular exogenous mechanism turns out to be order independent. However, the class of order-independent mechanisms, which also involve some practical mechanisms, is far richer than the random-order mechanism.

We will next characterize all order-independent mechanisms in the regular rounds. We will then show that there are uncountably many such mechanisms in Section 6.2.

We first introduce a class of mechanisms that will be crucial in our analysis of order-independent mechanisms. A mechanism $\phi$ is uneven score symmetric if for all $\left(h^{k-1} ; g_{T_{1}}: g_{T_{2}}\right)$ and $\left(h^{\prime k-1} ; g_{T_{2}}\right.$ : $\left.g_{T_{1}}\right)$ such that $g_{T_{1}} \neq g_{T_{2}}$ and $k \leq n$, we have $\phi\left(h^{k-1} ; g_{T_{1}}: g_{T_{2}}\right)=1-\phi\left(h^{\prime k-1} ; g_{T_{2}}: g_{T_{1}}\right)$. That is, as long as the score is not tied at the end of a round, the probability of who kicks first in the next round is the same for Team 1 and Team 2 whenever they are in each other's shoes. E.g., when Team 1 is ahead $1-0$ in (the beginning of) Round 2, and when Team 2 is ahead in Round 2 with a score of $0-1$, in Round 2 Team 1's probability of kicking first in the first case is the same as Team 2's probability of kicking first in the second case.

It turns out that such mechanisms fully characterize the order-independent mechanisms in the regular rounds.

Theorem 1 (Order-independent mechanisms in regular rounds) Suppose a mechanism $\phi$ is order independent in sudden-death rounds. $\phi$ is order independent in regular rounds if and only if it is uneven score symmetric.

When a regular round starts with an even score, the first team's kicker and the second team's kicker both exert the same effort and kick to the same intended spot. This is almost like asserting that when the score is even, kicking order is of minimum importance. The importance of kicking order, on the other hand, stems from the fact that when the score is uneven at the beginning of a round, kickers assert different levels of effort in their kicks depending on when they kick. Under an uneven score symmetric mechanism, each team's kickers foresee that their team will be treated symmetrically as the other team, in case either team falls behind or jumps ahead in score. Therefore, this assurance in essence takes the reason behind the importance of kicking order out of the equation.

A corollary to the proof of this theorem is of independent interest. It tells how effort levels can be compared between the teams in different states and rounds (see also Figure 1). 


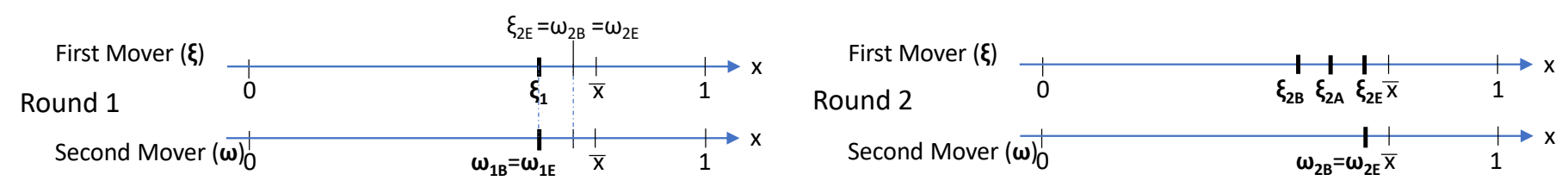

Figure 1: The effort levels of teams under an order-independent mechanism is state-symmetric equilibria in an easy shootout.

Corollary 1 Let $\phi$ be an order-independent mechanism in regular rounds. Suppose effort levels, i.e., the state-symmetric equilibrium intended spots, are denoted as follows:

- In Round 1: (1) for first kicking team as $\xi_{1}$; and (2) for second kicking team as $\omega_{1 E}$ when score is even and as $\omega_{1 B}$ when it is behind.

- In Round 2: (1) for first kicking team as $\xi_{2 A}$ when it is ahead, as $\xi_{2 E}$ when the score is even, and as $\xi_{2 B}$ when it is behind; and (2) for second kicking team as $\omega_{2 E}$ when score is even and as $\omega_{2 B}$ when it is behind.

Then the following relations hold for easy shootouts:

Round 1. $\xi_{1}=\omega_{1 E}=\omega_{1 B}<\omega_{2 B}$.

Round 2. $\xi_{2 B}<\xi_{2 A}<\xi_{2 E}=\omega_{2 E}=\omega_{2 B}$.

For difficult shootouts, only the first inequality reverses in Round 2 relations above.

Next we provide the intuition behind our characterization in Theorem 1 (with also Proposition 1 and Corollary 1). The difference in kickers' best responses in a round stems from their different marginal contribution to their team's winning chances. The marginal contribution of a kicker is the difference between his team's expected payoff in cases he scores a goal and fails to score. Suppose teams enter the last regular round tied. Moreover, suppose order independence is sustained in sudden-death rounds: if there is a tie after the last regular round, each team wins with probability $\frac{1}{2}$ at the beginning of sudden-death rounds. Then the best response of the first-kicking team's player, who we refer to as Kicker 1, turns out to be identical to that of the second-kicking team's player, Kicker 2. This is because these two scenarios lead to the same marginal contributions for both kickers, although this may not look obvious. We explain the reason for this in the next few paragraphs.

For Kicker 2, after a failed goal attempt by Kicker 1, the probability of his team winning after Kicker 2's goal is 1, while the probability of his team winning after his miss is $\frac{1}{2}$ with the marginal contribution in terms of probabilities as $1-\frac{1}{2}=\frac{1}{2}$. The same marginal contribution for Kicker 2 holds, if Kicker 1 scores and Kicker 2's team falls behind: If Kicker 2 scores, the shootout goes to sudden death and his team wins with probability $\frac{1}{2}$; if he misses, his team loses for sure, resulting with a marginal contribution of winning probability $\frac{1}{2}$. 
On the other hand, for Kicker 1, if he scores, the probability of his team winning is the probability Kicker 2 misses (let's say this occurs with probability $p$ ) plus Kicker 2 scores (with probability $(1-p)$ ), and then with probability $\frac{1}{2}$ Kicker 1 's team wins (as the game goes to sudden death with a tie). If he fails to score, the probability of his team winning is the probability Kicker 2 misses (with the same probability $p$, as we showed above Kicker 2 uses the same strategy in both cases) and with probability $\frac{1}{2}$ Kicker 1's team wins in sudden death. The difference between these two probabilities is $p+\frac{1}{2}(1-p)-\frac{1}{2} p=\frac{1}{2}$, and this is Kicker 1's marginal contribution to his team winning. Thus, Kickers 1 and 2 use the same strategies in any event in the last regular round when it starts with a tie. This leads to equal chances to win for both teams.

However, if the score were not tied at the beginning of the last regular round, say for example, Kicker 1's team were ahead, he would be less aggressive compared to when the score were tied. This is because his maximum marginal contribution to winning would be less than $\frac{1}{2}$ (as there is a more than $\frac{1}{2}$ probability his team will win anyway even if he misses, and for sure if he scores). As a result he does not take the shot with a high risk of the ball going out, although it may result with a goal with a higher probability. The case turns out to be even more dire if the first-kicking team is behind in the last round: Kicker 1's maximum marginal contribution is even smaller than the case when his team is ahead (this requires more in depth calculation to illustrate).

Therefore, Kicker 1 of the last regular round has decreasing accuracy in his shot in the order of the states for his team as tie, being ahead, and being behind. On the other hand, the states and strategies are identical for Kicker 2 as the case when that round started tied. Therefore, Kickers 1 and 2 use different strategies in the last regular round if the round does not start with a tie.

Next consider penultimate-round kickers when this round starts with a tie. We refer the firstkicking team's player in this round as Kicker 1 and the other player as Kicker 2. By backward induction, consider Kicker 2. If the game is tied before he kicks, his contribution (i) from scoring is $p_{A}^{2}$, which is the probability that Kicker 2's team wins when it starts last round ahead and (ii) from missing is $\frac{1}{2}$, as the score will be tied in last round and by above intuition either team has equal chance of winning (for any mechanism that is order independent in sudden-death rounds when last regular round starts with a tie); so his marginal contribution is $p_{A}^{2}-\frac{1}{2}$. If Kicker 1's team is ahead before Kicker 2 kicks, his contribution (i) from scoring is $\frac{1}{2}$, as teams enter last round tied and (ii) from missing is $p_{B}^{2}$, which is the probability that Kicker 2's team wins when it starts last round behind; so his marginal contribution is $\frac{1}{2}-p_{B}^{2}$.

The crucial argument is that these two marginal contributions are equal if and only if the mechanism is uneven score symmetric. It follows from the fact that in any round the sum of probabilities of one team winning plus the other team winning is always 1 , because the shootout always ends with a winner. Thus, in the last round, if one team is ahead then the other team is behind, and hence, $p_{A}^{1}+p_{B}^{2}=1$, when Kicker 1 's team is ahead and $p_{A}^{1}$ is its winning probability, and $p_{B}^{1}+p_{A}^{2}=1$, when Kicker 1's team is behind and $p_{B}^{1}$ is its winning probability. Only in an uneven-score symmetric mechanism, $p_{B}^{1}=p_{B}^{2}$ and $p_{A}^{1}=p_{A}^{2}$ as both teams are treated symmetrically when they are ahead or behind. This follows from the fact that the last-round's first kicker is 
more aggressive when his team is ahead than behind as we explained previously. (It can also be shown that with a similar argument Kicker 1 of penultimate round has exactly the same marginal contribution as Kicker 2 when this round starts tied under uneven-score symmetric mechanisms.)

In particular, this is exactly why $\mathrm{ABAB}$ or $\mathrm{ABBA}$, or any fixed-order mechanism is not order independent: In the last round, as first kicking team is pre-determined and its kicker is more aggressive when it is ahead than behind, the equalities $p_{A}^{1}=p_{A}^{2}$ and $p_{B}^{1}=p_{B}^{2}$ do no longer hold (i.e., $\xi_{1}=\omega_{1 B}=\omega_{1 E}$ shown in Figure 1, no longer holds). Hence, even if the penultimate round starts tied, there are possible state-symmetric equilibria in which the kickers of this round will exert different efforts leading to different winning chances for their teams at the beginning of the penultimate round.

The theorem leads to another interesting point: There is only one class of order-independent exogenous mechanisms; the post-Round-1 random-order mechanisms that determine which team will kick first with an unbiased coin toss in each round after the first, while who goes first in Round 1 can be determined freely. We formalize it below, and it follows directly from Theorem 1.

Proposition 2 The class of post-Round-1 random-order mechanisms are the only exogenous mechanisms that are order independent.

Note that one does not need to treat both teams symmetrically all the time to obtain order independence. In fact, when the score is tied, it does not matter which team kicks first. However, when the score is not tied, teams need to be treated symmetrically. This feature opens the door for some interesting practical mechanisms to be order independent. Two subclasses of such mechanisms are the behind-first and ahead-first mechanisms. In behind first (ahead first), the team that is behind (ahead) in score after a round kicks first in the next round, and otherwise the order of the teams is determined in some other manner. There are also many other uneven score symmetric mechanisms in which lotteries play a significant role. For example, a lottery mechanism that forces the behind team to go first in $75 \%$ of the time and also Team 1 always to go first $60 \%$ of the time when the score is tied is also order independent.

Next we ask as the sudden-death rounds induce an infinite game, what do order-independent mechanisms look like in sudden-death rounds. It turns out that there order matters when the score is tied unlike in regular rounds.

\section{Sudden-death Rounds}

Sudden-death analysis is substantially different as regular-round analysis assumes that winning chances are equal after they are over and score is still tied, while sudden-death rounds make the game an infinite game and tries to analyze what actually the winning chances are after regular rounds.

Under ABAB or ABBA, one can have uneven scores, such as Team 1 being ahead, in an intermediate regular round. As we showed, however, they cannot satisfy uneven score symmetry of 
order-independent mechanisms in regular rounds. On the other hand, in the sudden-death rounds, the score is never uneven at the beginning of a round. Suppose sudden death is reached in ABAB and ABBA. Would they at least be order independent in sudden-death rounds? If not, what do sudden-death order-independent mechanisms look like? We start with ABAB to answer these questions.

\subsection{ABAB in Sudden-death Rounds}

We will now characterize the state-symmetric equilibria of ABAB in the sudden-death rounds. As the game is infinite now, we will pedantically take the reader through the kickers' dynamic problem as we did in Section 4 for a single round. Without loss of generality assume that Team 1 wins the coin toss before Round 1 and kicks first throughout.

At state-symmetric equilibria, if they exist, each Team 1 kicker will use exactly the same action when he kicks in the sudden-death rounds, as Team 1 always goes first and the score is tied at the beginning of each sudden-death round. Similarly, by symmetry, each Team 2 kicker will use exactly the same action when his team is behind (which can be by one goal at most), and he will use exactly the same action when the score is even (which can happen if the preceding Team 1 kicker kicks out or his kick is saved).

On the other hand, Team 1 and Team 2 kickers may potentially use different actions at statesymmetric equilibria, as they kick in different orders: in each round Team 1 goes first and Team 2 goes second. Hence, if a state-symmetric equilibrium exists, for a given $k=1,2$, the probability of Team $k$ winning is the same at the beginning of each sudden-death round.

At a state-symmetric equilibrium, let us define $V_{T_{1}}$ to be the value function of Team 1 , that is the expected utility it contributes by winning or losing to its all kickers, in the first sudden-death

round. Denote by $x$ the kicking strategy for Team 1's kickers. Define $V_{T_{2}}^{B}$ as the value function of Team 2 in the first sudden-death round when Team 2 is currently behind by one goal and $V_{T_{2}}^{E}$ as the value function of Team 2 in the first sudden-death round when the score is currently even. Team 2's kickers' optimal kicking strategy in each scenario is $y_{B}$ and $y_{E}$ respectively.

We can write the following Bellman equation for $V_{T_{1}}$ :

$$
V_{T_{1}}=P_{G}(x) W_{G, T_{1}}+\left[1-P_{G}(x)\right] W_{N G, T_{1}}
$$

where $W_{G, T_{1}}$ is the expected future value conditional on the kicker scoring and $W_{N G, T_{1}}$ is the expected future value conditional on the kicker not scoring. We have

$$
\begin{aligned}
W_{G, T_{1}} & =P_{G}\left(y_{B}\right) V_{T_{1}}+\left[1-P_{G}\left(y_{B}\right)\right] V_{W} \\
W_{N G, T_{1}} & =P_{G}\left(y_{E}\right) V_{L}+\left[1-P_{G}\left(y_{E}\right)\right] V_{T_{1}}
\end{aligned}
$$


For Team 2, we have

$$
\begin{gathered}
V_{T_{2}}^{B}=P_{G}\left(y_{B}\right) \underbrace{V_{T_{2}}}_{=W_{G, T_{2}}^{B}}+\left[1-P_{G}\left(y_{B}\right)\right] \underbrace{V_{L}}_{=W_{N G, T_{2}}^{B}} \\
V_{T_{2}}^{E}=P_{G}\left(y_{E}\right) \underbrace{V_{W}}_{=W_{G, T_{2}}^{E}}+\left[1-P_{G}\left(y_{E}\right)\right] \underbrace{V_{T_{2}}}_{=W_{N G, T_{2}}^{E}}
\end{gathered}
$$

where

$$
V_{T_{2}}=V_{W}+V_{L}-V_{T_{1}}
$$

is the continuation payoff attributed to Team 2 in our win-or-lose game.

Next, we solve the decision problem faced by each kicker given other players' actions and beliefs using the first-order necessary and sufficient conditions given in Equation 3. Recall that for a Kicker $\ell$

$$
P_{G}^{\prime}\left(x_{\ell}\right)\left(W_{G, \ell}-W_{N G, \ell}+U_{G}\right)+P_{O}^{\prime}\left(x_{\ell}\right) U_{O}=0
$$

where $x_{\ell}$ is the optimal spot for Kicker $\ell$.

We can then solve $\left(x, y_{B}, y_{E}\right)$ by plugging Equations 5 - 10 into Equation 11. To do that we need to resolve the continuation values $V_{T_{1}}$ and $V_{T_{2}}$ for each team.

From Equations 5, 6, and 7,

$$
V_{T_{1}}=\frac{P_{G}(x)\left[1-P_{G}\left(y_{B}\right)\right] V_{W}+\left[1-P_{G}(x)\right] P_{G}\left(y_{E}\right) V_{L}}{P_{G}(x)\left[1-P_{G}\left(y_{B}\right)\right]+\left[1-P_{G}(x)\right] P_{G}\left(y_{E}\right)}=\alpha V_{W}+(1-\alpha) V_{L}
$$

where the winning probability of $T_{1}, \alpha$, is given by

$$
\alpha=\frac{P_{G}(x)\left[1-P_{G}\left(y_{B}\right)\right]}{P_{G}(x)\left[1-P_{G}\left(y_{B}\right)\right]+\left[1-P_{G}(x)\right] P_{G}\left(y_{E}\right)} .
$$

A value for $\alpha>0.5$ at a state-symmetric equilibrium will signal that ABAB is biased in favor of the first-kicking team in the sudden-death rounds, and for $\alpha<0.5$ it is vice versa for the second-kicking team. On the other hand, ABAB is an order-independent mechanism if and only if $\alpha=0.5$ at every state-symmetric equilibrium. For Team 2, then, we get by Equation 10,

$$
V_{T_{2}}=(1-\alpha) V_{W}+\alpha V_{L}
$$

Hence, Equations 5 - 10 through Equation 11 become self-contained to solve for $x, y_{B}$ and $y_{E}$. The following theorem characterizes the state-symmetric equilibrium strategy candidates solving these equations:

Theorem 2 (ABAB in sudden-death rounds) In sudden-death rounds, a state-symmetric interior equilibrium of $A B A B$ exists if and only if $P_{G}^{\prime}(0)\left[\frac{V_{W}-V_{L}}{2}+U_{G}\right]+P_{O}^{\prime}(0) U_{O}>0 .{ }^{25}$

(ii) When it exists, all equilibria with strategy profiles $\left(x, y_{B}, y_{E}\right)$, all of which are to the left of the goal-optimal spot, satisfying

\footnotetext{
${ }^{25} \mathrm{An}$ interior equilibrium is an equilibrium where kicker-optimal spots are interior.
} 
- $x=y_{E}$, i.e., the Team 1 kicker and Team 2 kicker, when the score is even, kick at the same spot; and

- for every equilibrium with $\left(y_{E}, y_{B}\right)$, there exists another equilibrium with $\left(\hat{y}_{E}, \hat{y}_{B}\right)$ such that $\hat{y}_{E}=y_{B}$ and $\hat{y}_{B}=y_{E}$.

We quantify when multiple equilibria exist and what the equilibrium strategies are for $\mathrm{ABAB}$ in Appendix B, we also give a numerical example of a set of parameters that makes $\mathrm{ABAB}$ order dependent in sudden-death rounds.

We briefly explain the intuition behind Theorem 2. It turns out that shootout mechanisms may in general generate a dynamic-team version of the hawk-dove game in sudden-death rounds: Suppose Team 1, either the first-kicking or the second-kicking, has a more aggressive kicking strategy, involving more accurate kicks to score but riskier to kick out, with respect to Team 2. Suppose the same strategy is used by all of Team 1's players. Then, Team 2 will win with a smaller probability

than $\frac{1}{2}$. To see how this can be sustained in an equilibrium, consider one of Team 2's kickers and the round he takes his shot: If the score is tied again at the end of this round, his team will lose with a higher probability in the future because of his teammates' less aggressive strategies. Thus, given enough chance of the tie continuing at the end of this round, trying to kick more aggressively is costly for him, now, as he may kick the ball out with a higher probability. Given also the more aggressive behavior of Team 1's kicker in his round, the Team 2 kicker does not have an incentive to be aggressive. A similar argument can be made for Team 1 players' best response involving more aggressive strategies given the others' fixed strategies. Observe that ABAB imposes always a different situation being faced by the first-kicking and second-kicking team players. Thus, an exogenous symmetry requirement has no constraint on the strategies of two teams as in our state-symmetric equilibrium concept. Thus such equilibria survive symmetric-state treatments and a state-symmetric equilibrium involving unequal chances of winning for the teams is feasible.

We also show in Appendix $H$ that it is possible to devise a forward-looking equilibrium refinement - similar in vein to the Intuitive Criterion of Cho and Kreps (1987) - to get rid of the multiplicity of equilibria, so that the first-kicking team wins more often. Any potential equilibrium candidate with the second-mover advantage does not survive this refinement, however.

\subsection{ABBA and Order Independent Mechanisms in Sudden-death Rounds}

Next, we consider ABBA. Contrary to ABAB, ABBA is order independent in sudden-death rounds, although it is not in regular rounds. It turns out that, alternating the order creates an opportunity symmetry for the future causing this particular scheme to be order independent in sudden death.

Theorem 3 (ABBA in sudden-death rounds) $A B B A$ is order independent in sudden-death rounds. 
Actually, for such a restriction to hold, we do not even need the teams to be in each other's shoes as frequently as in ABBA. In fact, there are uncountably many other mechanisms that are order independent in sudden-death rounds:

Theorem 4 (Order-independent mechanisms) Take any mechanism $\phi$ and any orderindependent mechanism $\varphi$. Fix a Sudden-death Round $k$. Construct a mechanism $\psi$ such that for sudden-death rounds from the beginning until Round $k-1$ 's end, it uses $\phi$ 's order structure, and for any other round, it uses $\varphi$ 's order structure. Formally,

- for all $\ell$ such that $n<\ell<k$, feasible scores $g_{T_{1}}: g_{T_{2}}$, and beginning of Round $\ell$ kicking orders $h^{\ell-1}$, let $\psi\left(h^{\ell-1} ; g_{T_{1}}: g_{T_{2}}\right)=\phi\left(h^{\ell-1} ; g_{T_{1}}: g_{T_{2}}\right)$, and

- for all $\ell \geq k$ and $\ell \leq n$, feasible scores $g_{T_{1}}: g_{T_{2}}$, and beginning of Round $\ell$ kicking orders $h^{\ell-1}$, let $\psi\left(h^{\ell-1} ; g_{T_{1}}: g_{T_{2}}\right)=\varphi\left(h^{\ell-1} ; g_{T_{1}}: g_{T_{2}}\right)$.

Then $\psi$ is order independent.

We can use Theorem 4 recursively, to obtain a very large class of order-independent mechanisms. The intuition of this result is as follows: Take the last round before order independence kicks in, say Round $k$. By backward induction, as teams are tied at the beginning of Round $k$ and in Round $k+1$ they have a 50\% - 50\% chance of winning, in all situations the two kickers of Round $k$ exert the same effort regardless of kicking order (as we explained in the intuition behind Theorem 1). Therefore, at the beginning of Round $k$, both teams have an equal chance of winning as well. An example of such a mechanism is a behind-first mechanism such that in the first $n+10$ rounds Team 1 kicks first whenever the game is tied, and then we alternate the order. Note that in the first 10 sudden-death rounds Team 1 kicks first, and yet, the mechanism is order independent as it is appended by an order-independent mechanism in sudden-death rounds, namely ABBA.

Although state-symmetric equilibria of ABAB in which teams exert different effort are still equilibria of ABBA, these equilibria are no longer state-symmetric under ABBA: If Team 1 kickers always exert a higher effort than Team 2's in ABAB, now their position as first or second kickers will alternate in ABBA. Thus, when the state is "kicking first," if it is a Team 1 kicker then he will exert higher effort in the same state than Team 2 kicker, violating state symmetry.

\section{Discussion: Order Independence vs Procedural Fairness}

Order independence implies ex-ante fairness, and in our context they are both about the distribution of state-symmetric equilibria. The starting team can be determined by alphabetical order of the names of the teams and yet we can still obtain order independence. Thus, not only an even coin flip to determine which team will start first is not needed, the existence of such coin flip does not guarantee ex-ante fairness of the state-symmetric equilibrium outcomes. That is one other aspect ABAB fails: it is not even ex-ante fair in this sense. 
However, there is a certain appeal of procedural fairness that an even coin flip determines which team will start first. This appeal is not only aesthetic: procedural fairness matters, as there are clearly many other factors that are known to affect the outcome, maybe, unfairly. For example, performance under pressure is one that is investigated in both sports sciences (see Jordet et al., 2006 and Jordet, 2009) and in economics (see Apesteguia and Palacios-Huerta, 2010 and Vandebroek, McCann, and Vroom, 2018). Hence, the more we can equalize the stressful situations the teams face, the better it will be according to this criterion. The random-order exogenous mechanism, determining the team that will go first in each round by an even coin flip is both order independent and procedurally fair. However, it involves certain kinds of ex-post unfairness with non-negligible probabilities, such as a team kicking first in back-to-back rounds (more than 90\% chance) or one team kicking first at least in four rounds (more than 35\% chance) unrelated to the current score of the shootout. Moreover, it is rather impractical to implement during a penalty shootout.

A desirable shootout mechanism should answer above concerns appropriately as well. With that goal in mind, we are able to obtain a tight characterization using procedural fairness and order independence and other efficiency-oriented properties that we discussed together with a practical simplicity property in Appendix F. Here, we only summarize this characterization result (where the characterization and more elaboration on other properties too can be found in that appendix).

We start with a property that can be used to give the opportunity of kicking to as many players as possible. For easy contests, we show that there is a class of order-independent mechanisms satisfying maximization of the expected number of attempts, which we term the behind-first mechanisms, such that the team that is behind in score after a round always kicks first in the next round, but if the score is tied after any round, then any random or fixed exogenous or endogenous order is admissible at the next round. In difficult contests, the same property is satisfied by ahead-first mechanisms.

Note that order-independent mechanisms, including behind-first and ahead-first subclasses, leave unspecified how one should choose which team would kick first when the score is tied, which would be a major issue especially during the sudden-death rounds of a shootout. We use a procedural fairness property in sudden-death rounds as much as possible short of flipping a coin before every round (which we have already noted as impractical). One thing we showed is that ABAB is not order independent, and the order should somehow alter in sudden-death rounds. Hence, we propose to embed the ABBA structure to our behind-first and ahead-first mechanisms in sudden-death rounds. Although there are many other ways to obtain order independence by altering order in sudden-death rounds, this mechanism provides a procedural fairness notion that we dub as sudden-death equality of opportunity to both teams in addition to its practicality. This also addresses almost all fairness concerns of previous investigators regarding performance difficulties under stress criteria: One team kicks first at most one more or less time than the other team in stressful situations of kicking while behind (and equal times when the sudden-death rounds are even).

This change of order also naturally translates to the regular rounds with a formally defined simplicity property. Our simplicity property minimizes the patterns of how kicking order changes across rounds while keeping the probability of either team kicking first ex-ante positive. We uniquely 
characterize easy shootout mechanisms, such as the ones in football, satisfying order independence and maximization of the expected number of attempts together with the other two properties, namely, simplicity and sudden-death equality of opportunity: The team that is behind in score after a round always kicks first in the next round, but if the score is tied after any round, then the team that kicked second at that round kicks first in the next round. We refer to this mechanism the alternating-order behind-first mechanism. Symmetrically, in difficult shootouts, such as in hockey, its symmetric counterpart, alternating-order ahead first uniquely characterizes this class.

We state this theorem formally here while the properties are defined formally in Appendix F:

Theorem 5 (Practical order-independent mechanisms) In an easy shootout, alternating order behind first is the unique order-independent mechanism that maximizes the expected number of attempts and satisfies simplicity and sudden-death equality of opportunity.

On the other hand, in a difficult shootout, alternating-order ahead first is the unique orderindependent mechanism that maximizes the expected number of attempts and satisfies simplicity and sudden-death equality of opportunity. ${ }^{26}$

This characterization is agnostic about which team should start the shootout, as this is immaterial for the equilibrium outcome for endogenous order-independent mechanisms. We can add to this characterization one additional ex-ante procedural fairness notion: Each team should face the same sequence of orders ex ante. This motivates an even coin flip at the beginning to determine which team will kick first. Then, we have a unique practical mechanism to propose for each contest structure, easy-task or difficult-task. We summarize the properties of practical mechanisms we have uncovered so far in Table 1.

\begin{tabular}{c|c|c|c|c|c} 
Mechanism & $\begin{array}{c}\text { Procedurally } \\
\text { Fair }\end{array}$ & $\begin{array}{c}\text { Ex-ante } \\
\text { Fair }\end{array}$ & $\begin{array}{c}\text { Order Independent } \\
\text { in Regular Rounds }\end{array}$ & $\begin{array}{c}\text { Order Independent } \\
\text { in Sudden Death }\end{array}$ & $\begin{array}{c}\text { Order } \\
\text { Independent }\end{array}$ \\
\hline \hline ABAB & $\checkmark$ & $x$ & $x$ & $x$ & $x$ \\
\hline ABBA & $\checkmark$ & $\checkmark$ & $x$ & $\checkmark$ & $x$ \\
\hline $\begin{array}{c}\text { Alternating Order } \\
\text { Behind/Ahead First }\end{array}$ & $\checkmark$ & $\checkmark$ & $\checkmark$ & $\checkmark$ & $\checkmark$
\end{tabular}

Table 1: Properties of Mechanisms

To conclude, our model adds only one parameter to the standard dynamic tug-of-war framework. A kicker has different utility levels for saved or missed kicks, even though they are outcome equivalent from his team's perspective (which is supported by empirical evidence). This small nuance in the model generates rich results and explains certain stylized empirical facts of ABAB. Moreover, it helps us characterize order-independent shootout mechanisms in this setting. This is the first paper using rational economic theory to address this design issue, and it addresses many empirical

\footnotetext{
${ }^{26}$ Maximizing the expected number of attempts property only holds for $n=2$ regular rounds; thus, this particular characterization holds for $n=2$.
} 
abnormalities surprisingly well through our approach of one parameter deviation from a model of players with only outcome-oriented preferences. ${ }^{27}$

\section{A Proofs of Proposition 1, Theorem 1, Corollary 1}

Proof of Proposition 1. First observe that $\bar{x}$ solves Equation 4 when $U_{O}=0$. As the partial derivative w.r.t. $U_{O}$ on the (left-hand side of) first-order condition is $P_{O}^{\prime}\left(x_{\ell}^{*}\right)>0$, the implicit function theorem implies that $x_{\ell}^{*}<\bar{x}$. Moreover, as the partial derivative w.r.t. $W_{G, \ell}-W_{N G, \ell}$ on the first-order condition is $P_{G}^{\prime}\left(x_{\ell}^{*}\right)>0\left(\right.$ as $\left.x_{\ell}^{*}<\bar{x}\right)$, the implicit function theorem again implies that the higher the expected marginal contribution, $W_{G, \ell}-W_{N G, \ell}$, the higher is $x_{\ell}^{*}$; and the higher is $P_{G}\left(x_{\ell}^{*}\right)$.

Proof of Theorem 1. We will prove the theorem for easy shootouts for $n=2$. The proof is symmetric for difficult shootouts. For $n=3$, the proof is given in Appendix G. For $n=1$, the proof follows from Round 2's analysis below and all mechanisms are uneven score symmetric for $n=1$. Suppose $n=2$. We solve it by backward induction. As both teams have an equal chance of winning in sudden-death rounds, the value function is $\frac{V_{W}+V_{L}}{2}$ for each team at the end of the regular rounds.

Round 2, Second Kick. Whether the score is even or the last-kicking team is behind, it can readily be verified that the optimal kicking strategy is always $\xi<\bar{x}$, where $\xi$ is determined by the following first-order condition by Equation 3 where $W_{G, 2, T_{2}}-W_{N G, 2, T_{2}}=\frac{V_{W}-V_{L}}{2}$ is his marginal contribution (as explained in the main text):

$$
P_{G}^{\prime}(\xi)\left[\frac{V_{W}-V_{L}}{2}+U_{G}\right]+P_{O}^{\prime}(\xi) U_{O}=0
$$

Round 2, First Kick. Next we look at the optimal kicking strategy for the first-kicking team in Round 2. Consider two cases:

Case 1: When Team 2 kicks first in Round 2. There are three possible states: when the score is currently even, when Team 2 is currently behind (by one goal), and when Team 2 is currently ahead (by one goal).

- When the score is currently even: Let $y_{2 E}$ denote the optimal kicking strategy for Team 2's kicker in Round 2 when the score is even. The value function for Team 2 is

$$
\begin{aligned}
V_{T_{2}, P_{2}, E}=[ & \left.P_{G}\left(y_{2 E}\right) P_{G}(\xi)+\left(1-P_{G}\left(y_{2 E}\right)\right)\left(1-P_{G}(\xi)\right)\right] \frac{V_{W}+V_{L}}{2} \\
& +P_{G}\left(y_{2 E}\right)\left(1-P_{G}(\xi)\right) V_{W}+\left(1-P_{G}\left(y_{2 E}\right)\right) P_{G}(\xi) V_{L}
\end{aligned}
$$

\footnotetext{
${ }^{27}$ As pointed out by an anonymous referee, because the outcome of a single penalty kick is binary (score or not), risk and preferences over risk are irrelevant for a rational model to capture such first-order conditions.
} 
By Equation 11, $y_{2 E}$ solves the following first-order condition:

$$
\begin{aligned}
P_{G}^{\prime}\left(y_{2 E}\right)\left[\left[P_{G}(\xi)-\left(1-P_{G}(\xi)\right)\right] \frac{V_{W}+V_{L}}{2}+\right. & \left.\left(1-P_{G}(\xi)\right) V_{W}-P_{G}(\xi) V_{L}+U_{G}\right]+P_{O}^{\prime}\left(y_{2 E}\right) U_{O}=0 \\
& \Longrightarrow P_{G}^{\prime}\left(y_{2 E}\right)\left[\frac{V_{W}-V_{L}}{2}+U_{G}\right]+P_{O}^{\prime}\left(y_{2 E}\right) U_{O}=0
\end{aligned}
$$

Therefore,

$$
y_{2 E}=\xi
$$

and $V_{T_{2}, P_{2}, E}=\frac{V_{W}+V_{L}}{2}$.

- When Team 2 is currently behind: Let $y_{2 B}$ denote the optimal kicking strategy for Team 2's kicker in Round 2 when Team 2 is currently behind. The value function for Team 2 is

$$
V_{T_{2}, P_{2}, B}=P_{G}\left(y_{2 B}\right) P_{G}(\xi) V_{L}+P_{G}\left(y_{2 B}\right)\left(1-P_{G}(\xi)\right) \frac{V_{W}+V_{L}}{2}+\left(1-P_{G}\left(y_{2 B}\right)\right) V_{L}
$$

$y_{2 B}$ satisfies the following first-order condition:

$$
\begin{gathered}
P_{G}^{\prime}\left(y_{2 B}\right)\left[P_{G}(\xi) V_{L}+\left(1-P_{G}(\xi)\right) \frac{V_{W}+V_{L}}{2}-V_{L}+U_{G}\right]+P_{O}^{\prime}\left(y_{2 B}\right) U_{O}=0 \\
\Longrightarrow P_{G}^{\prime}\left(y_{2 B}\right)\left[\left(1-P_{G}(\xi)\right) \frac{V_{W}-V_{L}}{2}+U_{G}\right]+P_{O}^{\prime}\left(y_{2 B}\right) U_{O}=0
\end{gathered}
$$

- When Team 2 is currently ahead: Let $y_{2 A}$ denote the optimal kicking strategy for Team 2's kicker in Round 2 when Team 2 is currently ahead. The value function for Team 2 is

$$
V_{T_{2}, P_{2}, A}=P_{G}\left(y_{2 A}\right) V_{W}+\left(1-P_{G}\left(y_{2 A}\right)\right)\left[\left(1-P_{G}(\xi)\right) V_{W}+P_{G}(\xi) \frac{V_{W}+V_{L}}{2}\right]
$$

The optimal kicking strategy, $y_{2 A}$, satisfies the following first-order condition:

$$
\begin{aligned}
& P_{G}^{\prime}\left(y_{2 A}\right)\left[V_{W}-(1-\right.\left.\left.P_{G}(\xi)\right) V_{W}-P_{G}(\xi) \frac{V_{W}+V_{L}}{2}+U_{G}\right]+P_{O}^{\prime}\left(y_{2 A}\right) U_{O}=0 \\
& \Longrightarrow P_{G}^{\prime}\left(y_{2 A}\right)\left[P_{G}(\xi) \frac{V_{W}-V_{L}}{2}+U_{G}\right]+P_{O}^{\prime}\left(y_{2 A}\right) U_{O}=0
\end{aligned}
$$

As

$$
P_{G}(\xi)>\frac{1}{2} \Longrightarrow y_{2 A}>y_{2 B}
$$

Moreover, since $P_{G}(\xi)<1$, Equations 15 and 17 imply

$$
y_{2 A}<\xi
$$

Case 2: When Team 1 kicks first in Round 2. Let $x_{2 E}, x_{2 B}$, and $x_{2 A}$ denote the optimal kicking strategy for Team 1's kicker in Round 2 when the score is even, when Team 1 is behind, and when Team 1 is ahead respectively. By symmetry, we have the following results:

- When the score is currently even: The optimal kicking strategy is

$$
x_{2 E}=y_{2 E}=\xi,
$$


and the value function for Team 1 is $V_{T_{1}, P_{2}, E}=\frac{V_{W}+V_{L}}{2}$.

- When Team 1 is currently behind: The optimal kicking strategy is

$$
x_{2 B}=y_{2 B}<\xi
$$

and the value function for Team 1 is

$$
V_{T_{1}, P_{2}, B}=P_{G}\left(x_{2 B}\right) P_{G}(\xi) V_{L}+P_{G}\left(x_{2 B}\right)\left(1-P_{G}(\xi)\right) \frac{V_{W}+V_{L}}{2}+\left(1-P_{G}\left(x_{2 B}\right)\right) V_{L}
$$

- When Team 1 is currently ahead: The optimal kicking strategy is

$$
x_{2 B}<x_{2 A}=y_{2 A}<\xi
$$

and the value function for Team 1 is

$$
V_{T_{1}, P_{2}, A}=P_{G}\left(x_{2 A}\right) V_{W}+\left(1-P_{G}\left(x_{2 A}\right)\right)\left[\left(1-P_{G}(\xi)\right) V_{W}+P_{G}(\xi) \frac{V_{W}+V_{L}}{2}\right]
$$

Round 1, Second Kick. Next we study the second team's optimal kicking strategy in Round 1. There are two possible states:

- When Team 1 does not score in Round 1: The value function for Team 2 in this case is

$V_{T_{2}, P_{1}, E}=P_{G}\left(y_{1 E}\right)\left[\phi\left(T_{1} ; 0: 1\right)\left(V_{W}+V_{L}-V_{T_{1}, P_{2}, B}\right)+\left(1-\phi\left(T_{1} ; 0: 1\right)\right) V_{T_{2}, P_{2}, A}\right]+\left(1-P_{G}\left(y_{1 E}\right)\right) \frac{V_{W}+V_{L}}{2}$ where

$$
\begin{aligned}
V_{T_{1}, P_{2}, B} & =P_{G}\left(x_{2 B}\right) P_{G}(\xi) V_{L}+P_{G}\left(x_{2 B}\right)\left(1-P_{G}(\xi)\right) \frac{V_{W}+V_{L}}{2}+\left(1-P_{G}\left(x_{2 B}\right)\right) V_{L} \\
& =\frac{V_{W}+V_{L}}{2}-\left[1-P_{G}\left(x_{2 B}\right)\left(1-P_{G}(\xi)\right)\right] \frac{V_{W}-V_{L}}{2} \\
V_{T_{2}, P_{2}, A} & =P_{G}\left(y_{2 A}\right) V_{W}+\left(1-P_{G}\left(y_{2 A}\right)\right)\left[\left(1-P_{G}(\xi)\right) V_{W}+P_{G}(\xi) \frac{V_{W}+V_{L}}{2}\right] \\
& =\frac{V_{W}+V_{L}}{2}+\left[1-\left(1-P_{G}\left(y_{2 A}\right)\right) P_{G}(\xi)\right] \frac{V_{W}-V_{L}}{2}
\end{aligned}
$$

We substitute the equations of $V_{T_{1}, P_{2}, B}$ and $V_{T_{2}, P_{2}, A}$ into $V_{T_{2}, P_{1}, E}$ as follows:

$$
\begin{gathered}
V_{T_{2}, P_{1}, E}=\frac{V_{W}+V_{L}}{2}+P_{G}\left(y_{1 E}\right)\left[\phi\left(T_{1} ; 0: 1\right)\left[1-P_{G}\left(x_{2 B}\right)\left(1-P_{G}(\xi)\right)\right]\right. \\
\left.+\left(1-\phi\left(T_{1} ; 0: 1\right)\right)\left[1-\left(1-P_{G}\left(y_{2 A}\right)\right) P_{G}(\xi)\right]\right] \frac{V_{W}-V_{L}}{2}
\end{gathered}
$$

The optimal kicking strategy, $y_{1 E}$, satisfies the following first-order condition:

$$
\begin{gathered}
P_{G}^{\prime}\left(y_{1 E}\right)\left[\alpha_{1} \frac{V_{W}-V_{L}}{2}+U_{G}\right]+P_{O}^{\prime}\left(y_{1 E}\right) U_{O}=0, \text { where } \\
\alpha_{1}=\phi\left(T_{1} ; 0: 1\right)\left[1-P_{G}\left(x_{2 B}\right)\left(1-P_{G}(\xi)\right)\right]+\left(1-\phi\left(T_{1} ; 0: 1\right)\right)\left[1-\left(1-P_{G}\left(y_{2 A}\right)\right) P_{G}(\xi)\right] .
\end{gathered}
$$

- When Team 1 scores in Round 1: The value function for Team 2 is $V_{T_{2}, P_{1}, B}=P_{G}\left(y_{1 B}\right) \frac{V_{W}+V_{L}}{2}+\left(1-P_{G}\left(y_{1 B}\right)\right)\left[\left(1-\phi\left(T_{1} ; 1: 0\right)\right) V_{T_{2}, P_{2}, B}+\phi\left(T_{1} ; 1: 0\right)\left(V_{W}+V_{L}-V_{T_{1}, P_{2}, A}\right)\right]$ 
where

$$
\begin{aligned}
V_{T_{2}, P_{2}, B} & =P_{G}\left(y_{2 B}\right) P_{G}(\xi) V_{L}+P_{G}\left(y_{2 B}\right)\left(1-P_{G}(\xi)\right) \frac{V_{W}+V_{L}}{2}+\left(1-P_{G}\left(y_{2 B}\right)\right) V_{L} \\
& =\frac{V_{W}+V_{L}}{2}-\left[1-P_{G}\left(y_{2 B}\right)\left(1-P_{G}(\xi)\right)\right] \frac{V_{W}-V_{L}}{2} \\
V_{T_{1}, P_{2}, A} & =P_{G}\left(x_{2 A}\right) V_{W}+\left(1-P_{G}\left(x_{2 A}\right)\right)\left[\left(1-P_{G}(\xi)\right) V_{W}+P_{G}(\xi) \frac{V_{W}+V_{L}}{2}\right] \\
& =\frac{V_{W}+V_{L}}{2}+\left[1-\left(1-P_{G}\left(x_{2 A}\right)\right) P_{G}(\xi)\right] \frac{V_{W}-V_{L}}{2}
\end{aligned}
$$

We substitute the equations of $V_{T_{2}, P_{2}, B}$ and $V_{T_{1}, P_{2}, A}$ into $V_{T_{2}, P_{1}, B}$ as follows:

$$
\begin{aligned}
& V_{T_{2}, P_{1}, B}=\frac{V_{W}+V_{L}}{2}-\left(1-P_{G}\left(y_{1 B}\right)\right)\left[\left(1-\phi\left(T_{1} ; 1: 0\right)\right)\left[1-P_{G}\left(y_{2 B}\right)\left(1-P_{G}(\xi)\right)\right]\right. \\
& \left.+\phi\left(T_{1} ; 1: 0\right)\left[1-\left(1-P_{G}\left(x_{2 A}\right)\right) P_{G}(\xi)\right]\right] \frac{V_{W}-V_{L}}{2}
\end{aligned}
$$

The optimal kicking strategy, $y_{1 B}$, satisfies the following first-order condition:

$$
P_{G}^{\prime}\left(y_{1 B}\right)\left[\alpha_{2} \frac{V_{W}-V_{L}}{2}+U_{G}\right]+P_{O}^{\prime}\left(y_{1 B}\right) U_{O}=0,
$$

where

$$
\alpha_{2}=\left(1-\phi\left(T_{1} ; 1: 0\right)\right)\left[1-P_{G}\left(y_{2 B}\right)\left(1-P_{G}(\xi)\right)\right]+\phi\left(T_{1} ; 1: 0\right)\left[1-\left(1-P_{G}\left(x_{2 A}\right)\right) P_{G}(\xi)\right]
$$

Then $y_{1 B}=y_{1 E}$ iff $\alpha_{1}=\alpha_{2}$ iff

$$
\begin{aligned}
& \phi\left(T_{1} ; 0: 1\right)\left[1-P_{G}\left(x_{2 B}\right)\left(1-P_{G}(\xi)\right)\right]+\left(1-\phi\left(T_{1} ; 0: 1\right)\right)\left[1-\left(1-P_{G}\left(y_{2 A}\right)\right) P_{G}(\xi)\right] \\
& =\left(1-\phi\left(T_{1} ; 1: 0\right)\right)\left[1-P_{G}\left(y_{2 B}\right)\left(1-P_{G}(\xi)\right)\right]+\phi\left(T_{1} ; 1: 0\right)\left[1-\left(1-P_{G}\left(x_{2 A}\right)\right) P_{G}(\xi)\right] \\
\Longleftrightarrow & \left(1-\phi\left(T_{1} ; 0: 1\right)-\phi\left(T_{1} ; 1: 0\right)\right)\left[1-\left(1-P_{G}\left(y_{2 A}\right)\right) P_{G}(\xi)\right] \\
& =\left(1-\phi\left(T_{1} ; 0: 1\right)-\phi\left(T_{1} ; 1: 0\right)\right)\left[1-P_{G}\left(x_{2 B}\right)\left(1-P_{G}(\xi)\right)\right] \\
\Longleftrightarrow & \left(1-\phi\left(T_{1} ; 0: 1\right)-\phi\left(T_{1} ; 1: 0\right)\right)\left[\left(1-P_{G}\left(y_{2 A}\right)\right) P_{G}(\xi)-P_{G}\left(x_{2 B}\right)\left(1-P_{G}(\xi)\right)\right]=0
\end{aligned}
$$

However, $\left(1-P_{G}\left(y_{2 A}\right)\right) P_{G}(\xi)-P_{G}\left(x_{2 B}\right)\left(1-P_{G}(\xi)\right)>0$ as $\bar{x}>\xi>x_{2 B}$ and $y_{2 A}<\xi$. Accordingly,

$$
y_{1 B}=y_{1 E} \Longleftrightarrow \phi\left(T_{1} ; 0: 1\right)+\phi\left(T_{1} ; 1: 0\right)=1
$$

Round 1, First Kick. Finally, we solve for Team 1's optimal kicking strategy in Round 1. The value function for Team 1 is

$$
\begin{aligned}
V_{T_{1}} & =P_{G}\left(x_{1}\right)\left[V_{W}+V_{L}-V_{T_{2}, P_{1}, B}\right]+\left(1-P_{G}\left(x_{1}\right)\right)\left[V_{W}+V_{L}-V_{T_{2}, P_{1}, E}\right] \\
& =V_{W}+V_{L}-P_{G}\left(x_{1}\right) V_{T_{2}, P_{1}, B}-\left(1-P_{G}\left(x_{1}\right)\right) V_{T_{2}, P_{1}, E}
\end{aligned}
$$

We substitute the equations of $V_{T_{2}, P_{1}, B}$ and $V_{T_{2}, P_{1}, E}$ into $V_{T_{1}}$ as follows:

$$
V_{T_{1}}=\frac{V_{W}+V_{L}}{2}+\left[P_{G}\left(x_{1}\right)\left(1-P_{G}\left(y_{1 B}\right)\right) \alpha_{2}-\left(1-P_{G}\left(x_{1}\right)\right) P_{G}\left(y_{1 E}\right) \alpha_{1}\right] \frac{V_{W}-V_{L}}{2}
$$


The optimal kicking strategy, $x_{1}$, satisfies the following first-order condition:

$$
P_{G}^{\prime}\left(x_{1}\right)\left[\left[\left(1-P_{G}\left(y_{1 B}\right)\right) \alpha_{2}+P_{G}\left(y_{1 E}\right) \alpha_{1}\right] \frac{V_{W}-V_{L}}{2}+U_{G}\right]+P_{O}^{\prime}\left(x_{1}\right) U_{O}=0
$$

Therefore

$$
x_{1} \gtreqless y_{1 E} \Longleftrightarrow\left(1-P_{G}\left(y_{1 B}\right)\right) \alpha_{2} \gtreqless\left(1-P_{G}\left(y_{1 E}\right)\right) \alpha_{1}
$$

On the other hand, we have

$$
V_{T_{1}}=\frac{V_{W}+V_{L}}{2} \Longleftrightarrow P_{G}\left(x_{1}\right)\left(1-P_{G}\left(y_{1 B}\right)\right) \alpha_{2}=\left(1-P_{G}\left(x_{1}\right)\right) P_{G}\left(y_{1 E}\right) \alpha_{1}
$$

Given that both teams have an equal chance of winning in sudden-death rounds and $V_{T_{2}, P_{2}, E}=$ $V_{T_{1}, P_{2}, E}=\frac{V_{W}+V_{L}}{2}, \phi$ is order independent if and only if $V_{T_{1}}=\frac{V_{W}+V_{L}}{2}$. We first make the following claim:

Claim 1. $P_{G}\left(x_{1}\right)\left(1-P_{G}\left(y_{1 B}\right)\right) \alpha_{2}=\left(1-P_{G}\left(x_{1}\right)\right) P_{G}\left(y_{1 E}\right) \alpha_{1}$ if and only if $\left(1-P_{G}\left(y_{1 B}\right)\right) \alpha_{2}=$ $\left(1-P_{G}\left(y_{1 E}\right)\right) \alpha_{1}$.

Proof of Claim 1. ( $\Longrightarrow)$ Suppose to the contrary that $\left(1-P_{G}\left(y_{1 B}\right)\right) \alpha_{2} \neq\left(1-P_{G}\left(y_{1 E}\right)\right) \alpha_{1}$ but $P_{G}\left(x_{1}\right)\left(1-P_{G}\left(y_{1 B}\right)\right) \alpha_{2}=\left(1-P_{G}\left(x_{1}\right)\right) P_{G}\left(y_{1 E}\right) \alpha_{1}$. If $\left(1-P_{G}\left(y_{1 B}\right)\right) \alpha_{2}>\left(1-P_{G}\left(y_{1 E}\right)\right) \alpha_{1}$, then from the first-order condition of $x_{1}$ we have $\bar{x}>x_{1}>y_{1 E}$. Then $P_{G}\left(x_{1}\right)\left(1-P_{G}\left(y_{1 B}\right)\right) \alpha_{2}>$ $P_{G}\left(y_{1 E}\right)\left(1-P_{G}\left(y_{1 B}\right)\right) \alpha_{2}>P_{G}\left(y_{1 E}\right)\left(1-P_{G}\left(y_{1 E}\right)\right) \alpha_{1}>\left(1-P_{G}\left(x_{1}\right)\right) P_{G}\left(y_{1 E}\right) \alpha_{1}$, a contradiction. The other case can be analyzed in a similar fashion.

$(\Longleftarrow)$ If $\left(1-P_{G}\left(y_{1 B}\right)\right) \alpha_{2}=\left(1-P_{G}\left(y_{1 E}\right)\right) \alpha_{1}$, then from the first-order condition of $x_{1}$ we have $x_{1}=y_{1 E}$, which in turn implies

$P_{G}\left(x_{1}\right)\left(1-P_{G}\left(y_{1 B}\right)\right) \alpha_{2}=P_{G}\left(y_{1 E}\right)\left(1-P_{G}\left(y_{1 B}\right)\right) \alpha_{2}=P_{G}\left(y_{1 E}\right)\left(1-P_{G}\left(y_{1 E}\right)\right) \alpha_{1}=\left(1-P_{G}\left(x_{1}\right)\right) P_{G}\left(y_{1 E}\right) \alpha_{1}$

Hence the Claim is established. $\diamond$

Accordingly, $\phi$ is order independent if and only if

$$
\left(1-P_{G}\left(y_{1 B}\right)\right) \alpha_{2}=\left(1-P_{G}\left(y_{1 E}\right)\right) \alpha_{1} .
$$

This equality holds for an arbitrary pair of (feasible) probabilities, $\left\{P_{G}, P_{O}\right\}$, if and only if $\alpha_{1}=\alpha_{2}$, which holds if and only if $\phi\left(T_{1} ; 0: 1\right)+\phi\left(T_{1} ; 1: 0\right)=1$, i.e., $\phi$ is uneven score symmetric.

Proof of Corollary 1. It can readily be seen from the proof of Theorem 1 that the optimal kicking strategy at that state is solely determined by the state (the score difference and the kicking order in Round 2), and hence is independent of which order-independent mechanism leads to that state. W.l.o.g., suppose Team 1 moves first in Round 1 and Team 2 moves second. Consider Round 1 first. Equation 24 in the proof of Theorem 1 implies that $\omega_{1 E}=\omega_{1 B}$. Equations 25 and 26 imply that $\xi_{1}=\omega_{1 E}$. Next consider Round 2. Equation 15 implies that $\omega_{2 B}=\omega_{2 E}$. Equations 15 and 16 when Team 1 moves second in Round 2 and Equation 20 when Team 1 moves first in Round 1 imply that $\xi_{2 E}=\omega_{2 E}$. For an easy shootout, Equations 18 and 19 when Team 1 moves second in Round 2 and Equations 21 and 22 when Team 1 moves first in Round 2 imply that $\xi_{2 E}>\xi_{2 A}>\xi_{2 B}$. (where easiness of the shootout is only used in Equation 18, for a difficult shootout, we would have $\left.\xi_{2 E}>\xi_{2 B}>\xi_{2 A}\right)$. Finally, Equations 15 and 23 and the fact that $\alpha_{2}<1$ imply $\omega_{1 B}<\omega_{2 B}$. 


\section{B Order Dependence of ABAB in Sudden-death Rounds}

Theorem 6 (Order dependence of ABAB in sudden-death rounds) Suppose that in the sudden-death rounds of $A B A B$ a state-symmetric interior equilibrium exists. Then

- Multiple state-symmetric equilibria exist if and only if there are multiple solutions $\beta$ to the equation

$$
\Upsilon(\beta) \equiv \beta-\frac{1-P_{G}(y(1-\beta))}{2-P_{G}(y(\beta))-P_{G}(y(1-\beta))}=0,
$$

where $y(\beta)=f^{-1}\left(\frac{-U_{O}}{\left(V_{W}-V_{L}\right) \beta+U_{G}}\right)$ for $f(x)=P_{G}^{\prime}(x) / P_{O}^{\prime}(x)$ for all $x \in[0,1]$.

- $\Upsilon(\beta)=0$ has multiple solutions if

$$
\frac{U_{G}}{V_{W}-V_{L}}<\left.\frac{\left(\ln \left(1-P_{G}(x)\right)\right)^{\prime}}{2(\ln f(x))^{\prime}}\right|_{x=y\left(\frac{1}{2}\right)}-\frac{1}{2} .
$$

- There is an odd number of solutions with $\beta=\frac{1}{2}$ always being a solution and others being located symmetrically around it. We also have $y_{B}=y(\beta)$ and $x=y_{E}=y(1-\beta)$ for any solution $\beta$.

- $A B A B$ is not order independent in sudden-death rounds if and only if there are multiple statesymmetric equilibria of $A B A B$ in sudden-death rounds.

We can interpret the condition in Equation 27 as follows. If we rewrite the definition of $y(\beta)$ we get

$$
-U_{O} P_{O}^{\prime}(y(\beta))=P_{G}^{\prime}(y(\beta))\left(\left(V_{W}-V_{L}\right) \beta+U_{G}\right) .
$$

Here, the left hand side is the expected marginal disutility of kicking out for a player if $\beta$ is his team's continuation winning probability and his effort is accordingly $y(\beta)$. The right hand side is the expected marginal benefit for the same player from scoring; recall that $\left(V_{W}-V_{L}\right) \beta+U_{G}$ is the marginal benefit for the player from scoring. Thus, this equation is nothing but the first-order condition for a player to maximize his expected payoff.

When Team 1 wins with probability $\beta$, then Team 2 wins with probability $1-\beta$. We refer to $y(1-\beta)$ as the state-symmetric equilibrium effort of Kicker 2 in sudden death if his team is behind. Also we had shown in Theorem 1 and explained aftewards that when the score is even before Kicker 2 moves in the last regular round or in any sudden-death round, the effort of Kicker 2 is equal to the effort of Kicker 1. Then the winning probability $\alpha$ defined in Equation 13 boils down to Equation 27 for $\alpha=\beta$. Automatically $\beta=\frac{1}{2}$ solves this equation, and each team wins the shootout with equal probability in sudden death. But there can be asymmetric solutions for $\beta<\frac{1}{2}$ and $\beta>\frac{1}{2}$; moreover directly whenever $\beta=p$ solves it then $\beta=1-p$ also solves it. 
Thus, ABAB is not order independent as the winning probability of Team $1, \beta \neq \frac{1}{2}$ in equilibrium, whenever $y_{B} \neq y_{E}$.

On the other hand, the sufficiency condition in Equation 28 is a technical condition. The following example satisfies this condition and has multiple state-symmetric equilibria.

Example 1 (Order dependent equilibria) Suppose the game has the following structure:

$$
\begin{aligned}
V_{W}-V_{L} & =5.12 ; U_{G}=1 ; U_{O}=-28 \\
P_{G}(x) & =0.935-1.5(0.5-x)^{2} ; \quad P_{O}(x)=0.12 x+\mathbf{1}_{x \geq 0.54} 1.5(x-0.54)^{2},
\end{aligned}
$$

where $\mathbf{1}_{x \geq 0.53}$ is an indicator function. It can be readily verified that $\left(x, y_{E}, y_{B}\right)=$ $(0.2020,0.2020,0.1667)$ with $\beta=0.5388$ (and hence $\left(x, y_{E}, y_{B}\right)=(0.1667,0.1667,0.2020)$ with $\beta=0.4611)$ constitutes an equilibrium. On the other hand $\left(x, y_{E}, y_{B}\right)=(0.1853,0.1853,0.1853)$ is the equilibrium in which both teams have equal winning chance. ${ }^{28}$

\section{References}

[1] Almeida, C. H., A. Volossovitch, and R. Duarte. 2016. "Penalty Kick Outcomes in UEFA Club Competitions (2010-2015): The Roles of Situational, Individual and Performance Factors." International Journal of Performance Analysis in Sport, 16, 508-522.

[2] Anbarcı, N., C.-J Sun, and M. U. Ünver. 2015. "Designing Fair Tiebreak Mechanisms: The Case of FIFA Penalty Shootouts." http://dx.doi.org/10.2139/ssrn.2558979

[3] Apesteguia, J., and I. Palacios-Huerta. 2010. "Psychological Pressure in Competitive Environments: Evidence from a Randomized Natural Experiment." American Economic Review, 100, 2548-2564.

[4] Baggio, R. 2001. Una Porta Nel Cielo: Un'autobiografia. Limina (for quote in the paper see: http: //www. theguardian.com/sport/2002/may/19/worldcupfootbal12002.football).

[5] Bar-Eli, M., and O. H. Azar. 2009. "Penalty Kicks in Soccer: An Empirical Analysis of Shooting Strategies and Goalkeepers' Preferences." Soccer and Society, 10, 183-191.

[6] Bar-Eli, M., O. H. Azar, and Y. Lurie. 2009. “(Ir)rationality in Action: Do soccer Players and Goalkeepers Fail to Learn How to Best Perform during a Penalty Kick?" Progress in Brain Research, 174, 97-108.

[7] Bergantinos G. and J. D. Moreno-Ternero. 2018. "Sharing the Revenues from Broadcasting Sport Events." Working paper.

\footnotetext{
${ }^{28}$ We thank Randy Silvers for his help in generating the example.
} 
[8] Boczoń, M. and A. J. Wilson. 2018. "Goals, Constraints, and Public Assignment: A Field Study of the UEFA Champions Leauge." Working paper.

[9] Brams, S. J., M. Ismail, D. M. Kilgour, and W. Stromquist. 2018. "Catch-Up: A Rule that Makes Service Sports More Competitive." The American Mathematical Monthly, 125, 771-796.

[10] Brams, S. J. and M. Ismail. 2018. "Making the Rules of Sports Fairer." SIAM Review, 60, 181-202.

[11] Cabral, L. M. B. 2002. "Increasing Dominance with No Efficiency Effect." Journal of Economic Theory, 102, 471-479.

[12] Chapsal, A. and J.-B. Vilain. 2019. "Individual Contribution in Team Contests." Journal of Economic Psychology, 75, Part B, 102087.

[13] Che, Y.-K. and T. Hendershott. 2008. "How to Divide the Possession of a Football?" Economics Letters, 99, 561-565.

[14] Chiappori, P.-A., S. Levitt, and T. Groseclose. 2002. "Testing Mixed-Strategy Equilibria When Players Are Heterogeneous: The Case of Penalty Kicks in Soccer." American Economic Review, 92, 1138-1151.

[15] Cho, I.-K. and D. M. Kreps. 1987. "Signaling Games and Stable Equilibria." The Quarterly Journal of Economics, 102(2), 179-221.

[16] Cohen-Zada, D., A. Krumer, and O. M. Shapir. 2018. "Testing the Effect of Serve Order in Tennis Tiebreak." Journal of Economic Behavior and Organization, 146, 106-115.

[17] Csató, L. 2020. "A Comparison of Penalty Shootout Designs in Soccer." 4OR - A Quarterly Journal of Operations Research. https://doi.org/10.1007/s10288-020-00439-w.

[18] Echenique, F. 2017. "ABAB or ABBA? The Arithmetics of Penalty Shootouts in Soccer." CalTech, mimeo.

[19] Frechétte, G., A. E. Roth, and M. U. Ünver. 2007. "Unraveling Yields Inefficient Matching: Evidence from Post-Season College Football Bowls." RAND Journal of Economics, 38(4), 967982.

[20] Fudenberg, D., G. Richard, J. Stiglitz, and J. Tirole. 1983. "Preemption, Leapfrogging, and Competition in Patent Races." European Economic Review, 22, 3-31.

[21] Ginsburgh, V. A. and J. C. Van Ours. 2003. "Expert Opinion and Compensation: Evidence from a Musical Competition." American Economic Review, 93, 289-296. 
[22] González-Díaz, J. and I. Palacios-Huerta. 2016. "Cognitive Performance in Competitive Environments: Evidence from a Natural Experiment." Journal of Public Economics, 139, 40-52.

[23] Harford, T. 2006. "World Cup Game Theory: What Economics Tells Us about Penalty Kicks." The Undercover Economist, http://www.slate.com/articles/arts/the_ undercover_economist/2006/06/world_cup_game_theory.html.

[24] Harris, C. and J. Vickers. 1985. "Perfect Equilibrium in a Model of a Race." Review of Economic Studies, 52, 193-209.

[25] Harris, C. and J. Vickers. 1987. "Racing with Uncertainty." Review of Economic Studies, 54, 1-21.

[26] Jordet, G. 2009. "Why Do English Players Fail in Soccer Penalty Shootouts? A Study of Team Status, Self-Regulation, and Choking under Pressure." Journal of Sports Sciences, 27, 97-106.

[27] Jordet, G., E. Hartman, C. Visscher, and K. A. P. M. Lemmink. 2006. "Kicks from the Penalty Mark in Soccer: The Roles of Stress, Skill, and Fatigue for Kick Outcomes." Journal of Sports Sciences, 25, 121-129.

[28] Klumpp, T. and M. K. Polborn. 2006. "Primaries and the New Hampshire Effect." Journal of Public Economics, 90, 1073-1114.

[29] Kocher, M., M. Lenz, and M. Sutter. 2012. "Psychological Pressure in Competitive Environments: New Evidence from Randomized Natural Experiments." Management Science, 58, $1585-1591$.

[30] Palacios-Huerta, I. 2003. "Professionals Play Minimax." Review of Economic Studies, 70, 395-415.

[31] Palacios-Huerta, I. 2014. Beautiful Game Theory: How Soccer Can Help Economics. Princeton University Press.

[32] Rosen, S., 1986. "Prizes and Incentives in Elimination Tournaments." American Economic Review, 76, 701-715.

[33] Rubinstein, A. 1998. Modeling Bounded Rationality. MIT Press (cited in the Online Appendices)

[34] Szymanski, S. 2003. "The Economic Design of Sporting Contests." Journal of Economic Literature, $41,1137-1187$.

[35] Vandebroek, T. P., B. T. McCann, and G. Vroom. 2018. "Modeling the Effects of Psychological Pressure on First-Mover Advantage in Competitive Interactions: The Case of Penalty ShootOuts." Journal of Sports Economics, 19, 725-754. 


\section{Online Appendices for \\ "Designing Practical and Fair Sequential Team Contests" by Nejat Anbarcı, Ching-Jen Sun, \& M. Utku Ünver}

\section{Remaining Proofs of Results}

\section{Proof of Theorem 2.}

We write the three first-order conditions using Equation 11 (or 3) as:

$$
\begin{gathered}
P_{G}^{\prime}(x)\left[P_{G}\left(y_{B}\right) V_{T_{1}}+\left(1-P_{G}\left(y_{B}\right)\right) V_{W}-P_{G}\left(y_{E}\right) V_{L}-\left(1-P_{G}\left(y_{E}\right)\right) V_{T_{1}}+U_{G}\right]+P_{O}^{\prime}(x) U_{O}=0 \\
P_{G}^{\prime}\left(y_{B}\right)\left[V_{T_{2}}-V_{L}+U_{G}\right]+P_{O}^{\prime}\left(y_{B}\right) U_{O}=0 \\
P_{G}^{\prime}\left(y_{E}\right)\left[V_{W}-V_{T_{2}}+U_{G}\right]+P_{O}^{\prime}\left(y_{E}\right) U_{O}=0
\end{gathered}
$$

We first prove that $x=y_{E}$ in any state-symmetric equilibrium.

Claim 1. $x=y_{E}$.

Proof of Claim 1. Define

$$
\Delta=P_{G}\left(y_{B}\right) V_{T_{1}}+\left(1-P_{G}\left(y_{B}\right)\right) V_{W}-P_{G}\left(y_{E}\right) V_{L}-\left[1-P_{G}\left(y_{E}\right)\right] V_{T_{1}}-V_{W}+V_{T_{2}} .
$$

From the first-order conditions of $x$ and $y_{E}, x \geq y_{E}$ if and only if $\Delta \geq 0$. Recall that the winning probability of Team 1 in equilibrium, $\alpha$, is given in Equation 13. Hence,

$$
\begin{aligned}
\Delta & =P_{G}\left(y_{B}\right)\left(V_{T_{1}}-V_{W}\right)+P_{G}\left(y_{E}\right)\left(V_{T_{1}}-V_{L}\right)+V_{T_{2}}-V_{T_{1}} \\
& =P_{G}\left(y_{B}\right)(1-\alpha)\left(V_{L}-V_{W}\right)+P_{G}\left(y_{E}\right) \alpha\left(V_{W}-V_{L}\right)+(1-2 \alpha)\left(V_{W}-V_{L}\right) \\
& =\left[-P_{G}\left(y_{B}\right)(1-\alpha)+P_{G}\left(y_{E}\right) \alpha+1-2 \alpha\right]\left(V_{W}-V_{L}\right) \\
& =\left[1-P_{G}\left(y_{B}\right)+\left(P_{G}\left(y_{E}\right)+P_{G}\left(y_{B}\right)-2\right) \alpha\right]\left(V_{W}-V_{L}\right)
\end{aligned}
$$

We substitute $\alpha$ from Equation 13 as follows:

$$
\begin{aligned}
\Delta= & {\left[1-P_{G}\left(y_{B}\right)+\left(P_{G}\left(y_{E}\right)+P_{G}\left(y_{B}\right)-2\right) \frac{P_{G}(x)\left(1-P_{G}\left(y_{B}\right)\right)}{P_{G}(x)\left(1-P_{G}\left(y_{B}\right)\right)+\left(1-P_{G}(x)\right) P_{G}\left(y_{E}\right)}\right]\left(V_{W}-V_{L}\right) } \\
= & \left(1-P_{G}\left(y_{B}\right)\right)\left[1+\frac{\left(P_{G}\left(y_{E}\right)+P_{G}\left(y_{B}\right)-2\right) P_{G}(x)}{P_{G}(x)\left(1-P_{G}\left(y_{B}\right)\right)+\left(1-P_{G}(x)\right) P_{G}\left(y_{E}\right)}\right]\left(V_{W}-V_{L}\right) \\
= & {\left[\frac{\left(1-P_{G}\left(y_{B}\right)\right)\left(V_{W}-V_{L}\right)}{P_{G}(x)\left(1-P_{G}\left(y_{B}\right)\right)+\left(1-P_{G}(x)\right) P_{G}\left(y_{E}\right)}\right] } \\
& \times\left[P_{G}(x)\left(1-P_{G}\left(y_{B}\right)\right)+\left(1-P_{G}(x)\right) P_{G}\left(y_{E}\right)+\left(P_{G}\left(y_{E}\right)+P_{G}\left(y_{B}\right)-2\right) P_{G}(x)\right] \\
= & \frac{\left(1-P_{G}\left(y_{B}\right)\right)\left(V_{W}-V_{L}\right)}{P_{G}(x)\left(1-P_{G}\left(y_{B}\right)\right)+\left(1-P_{G}(x)\right) P_{G}\left(y_{E}\right)}\left[P_{G}\left(y_{E}\right)-P_{G}(x)\right]
\end{aligned}
$$


Suppose $x>y_{E}$, then as both $x, y_{E}<\bar{x}$ and $P_{G}$ is increasing on the left of $\bar{x}$, we have $P_{G}(x)>$ $P_{G}\left(y_{E}\right)$. But then $\Delta<0$, contradicting that $x>y_{E}$. Supposition $x<y_{E}$ leads to a similar contradiction. Therefore, we must have $x=y_{E}$. $\diamond$

Given $x=y_{E}, \alpha$ can be simplified as

$$
\alpha=\frac{P_{G}(x)\left(1-P_{G}\left(y_{B}\right)\right)}{P_{G}(x)\left(1-P_{G}\left(y_{B}\right)\right)+\left(1-P_{G}(x)\right) P_{G}\left(y_{E}\right)}=\frac{1-P_{G}\left(y_{B}\right)}{2-P_{G}\left(y_{B}\right)-P_{G}\left(y_{E}\right)},
$$

and $\alpha=\frac{1}{2}$ iff $x=y_{B}$. Then the first-order condition w.r.t. $y_{B}$ can be simplified as:

$$
\begin{array}{r}
P_{G}^{\prime}\left(y_{B}\right)\left[V_{T_{2}}-V_{L}+U_{G}\right]+P_{O}^{\prime}\left(y_{B}\right) U_{O}=0 \\
\Longrightarrow P_{G}^{\prime}\left(y_{B}\right)\left[(1-\alpha)\left(V_{W}-V_{L}\right)+U_{G}\right]+P_{O}^{\prime}\left(y_{B}\right) U_{O}=0 \\
\Longrightarrow P_{G}^{\prime}\left(y_{B}\right)\left[\left(V_{W}-V_{L}\right) \frac{1-P_{G}\left(y_{E}\right)}{2-P_{G}\left(y_{B}\right)-P_{G}\left(y_{E}\right)}+U_{G}\right]+P_{O}^{\prime}\left(y_{B}\right) U_{O}=0
\end{array}
$$

Similarly, the first-order condition w.r.t. $y_{E}$ can be simplified as:

$$
\begin{array}{r}
P_{G}^{\prime}\left(y_{E}\right)\left[V_{W}-V_{T_{2}}+U_{G}\right]+P_{O}^{\prime}\left(y_{E}\right) U_{O}=0 \\
\Longrightarrow P_{G}^{\prime}\left(y_{E}\right)\left[\alpha\left(V_{W}-V_{L}\right)+U_{G}\right]+P_{O}^{\prime}\left(y_{E}\right) U_{O}=0 \\
\Longrightarrow P_{G}^{\prime}\left(y_{E}\right)\left[\left(V_{W}-V_{L}\right) \frac{1-P_{G}\left(y_{B}\right)}{2-P_{G}\left(y_{B}\right)-P_{G}\left(y_{E}\right)}+U_{G}\right]+P_{O}^{\prime}\left(y_{E}\right) U_{O}=0
\end{array}
$$

Now we are ready to prove part (i). First we show that $P_{G}^{\prime}(0)\left[\frac{V_{W}-V_{L}}{2}+U_{G}\right]+P_{O}^{\prime}(0) U_{O}>0$ implies the existence of an interior equilibrium. Define $H(z) \equiv P_{G}^{\prime}(z)\left[\frac{V_{W}-V_{L}}{2}+U_{G}\right]+P_{O}^{\prime}(z) U_{O}$. H( $(z)$ is continuous with $H^{\prime}(z)<0$ as $P_{G}^{\prime \prime}(z)<0$ and $P_{O}^{\prime \prime}(z) \geq 0$. Then $H(0)=P_{G}^{\prime}(0)\left[\frac{V_{W}-V_{L}}{2}+U_{G}\right]+$ $P_{O}^{\prime}(0) U_{O}>0$ and $H(\bar{x})=P_{O}^{\prime}(\bar{x}) U_{O}<0$ implies that there exists some $a \in(0, \bar{x})$ such that $H(a)=0$. It can readily be seen that $\left(x, y_{B}, y_{E}\right)=(a, a, a)$ solves the two first-order conditions and hence constitutes an equilibrium. On the other hand, assume now $P_{G}^{\prime}(0)\left[\frac{V_{W}-V_{L}}{2}+U_{G}\right]+P_{O}^{\prime}(0) U_{O}=$ $H(0) \leq 0$. As $H^{\prime}(z)<0, H(z)<0$ for every $z \in[0,1]$. Suppose to the contrary that there exists an interior equilibrium $\left(x, y_{B}, y_{E}\right)$. Clearly $y_{B} \neq y_{E}$, for otherwise $\frac{1-P_{G}\left(y_{E}\right)}{2-P_{G}\left(y_{B}\right)-P_{G}\left(y_{E}\right)}=\frac{1}{2}$ and the first-order condition of $y_{B}$ becomes $H\left(y_{B}\right)<0$. Suppose $y_{B}>y_{E}$. Then the first-order condition w.r.t. $y_{E}$ in Equation 30 becomes:

$$
\begin{aligned}
& P_{G}^{\prime}\left(y_{E}\right)\left[\left(V_{W}-V_{L}\right) \frac{1-P_{G}\left(y_{B}\right)}{2-P_{G}\left(y_{B}\right)-P_{G}\left(y_{E}\right)}+U_{G}\right]+P_{O}^{\prime}\left(y_{E}\right) U_{O} \\
& <P_{G}^{\prime}\left(y_{E}\right)\left[\frac{V_{W}-V_{L}}{2}+U_{G}\right]+P_{O}^{\prime}\left(y_{E}\right) U_{O}=H\left(y_{E}\right)<0,
\end{aligned}
$$

a contradiction. Then $y_{B}<y_{E}$; and similarly, the first-order condition for $y_{B}$ is negative, leading to a contradiction. Therefore, an interior equilibrium exists if and only if $P_{G}^{\prime}\left[\frac{V_{W}-V_{L}}{2}+U_{G}\right]+$ $P_{O}^{\prime}(0) U_{O}=H(0)>0$. There may be multiple solutions $\left(y_{E}, y_{B}\right)$, and whenever one exists, then $\left(\hat{y_{E}}, \hat{y_{B}}\right)$ satisfying $\hat{y_{E}}=y_{B}$ and $\hat{y_{B}}=y_{E}$ also leads to a state-symmetric equilibrium.

Proof of Theorem 3. Without loss of generality, assume Team 1 kicks first in the first sudden-death round (i.e., in Round $n+1$ ). In a state-symmetric equilibrium, denote by $x_{I}$ the 
optimal kicking strategy for the first kicker in each sudden-death round, and $x_{B}\left(x_{E}\right)$ the optimal kicking strategy for the second kicker in each sudden-death round when the score is behind (tied). Let $V_{T_{1}}\left(V_{T_{2}}\right)$ denote Team 1's (Team 2's) value function at the beginning of the first sudden-death round (Round $n+1)$. Then

$$
\begin{aligned}
V_{T_{1}} & =\left[P_{G}\left(x_{I}\right) P_{G}\left(x_{B}\right)+\left(1-P_{G}\left(x_{I}\right)\right)\left(1-P_{G}\left(x_{E}\right)\right)\right] V_{T_{2}} \\
& +P_{G}\left(x_{I}\right)\left(1-P_{G}\left(x_{B}\right)\right) V_{W}+\left(1-P_{G}\left(x_{I}\right)\right) P_{G}\left(x_{E}\right) V_{L} \\
V_{T_{2}} & =\left[P_{G}\left(x_{I}\right) P_{G}\left(x_{B}\right)+\left(1-P_{G}\left(x_{I}\right)\right)\left(1-P_{G}\left(x_{E}\right)\right)\right] V_{T_{1}} \\
& +P_{G}\left(x_{I}\right)\left(1-P_{G}\left(x_{B}\right)\right) V_{L}+\left(1-P_{G}\left(x_{I}\right)\right) P_{G}\left(x_{E}\right) V_{W}
\end{aligned}
$$

We substitute $V_{T_{2}}$ into the equation of $V_{T_{1}}$ as follows:

$$
\begin{aligned}
V_{T_{1}} & =\left[P_{G}\left(x_{I}\right) P_{G}\left(x_{B}\right)+\left(1-P_{G}\left(x_{I}\right)\right)\left(1-P_{G}\left(x_{E}\right)\right)\right]^{2} V_{T_{1}} \\
& +\left[\left[P_{G}\left(x_{I}\right) P_{G}\left(x_{B}\right)+\left(1-P_{G}\left(x_{I}\right)\right)\left(1-P_{G}\left(x_{E}\right)\right)\right] P_{G}\left(x_{I}\right)\left(1-P_{G}\left(x_{B}\right)\right)+\left(1-P_{G}\left(x_{I}\right)\right) P_{G}\left(x_{E}\right)\right] V_{L} \\
& +\left[\left[P_{G}\left(x_{I}\right) P_{G}\left(x_{B}\right)+\left(1-P_{G}\left(x_{I}\right)\right)\left(1-P_{G}\left(x_{E}\right)\right)\right]\left(1-P_{G}\left(x_{I}\right)\right) P_{G}\left(x_{E}\right)+P_{G}\left(x_{I}\right)\left(1-P_{G}\left(x_{B}\right)\right)\right] V_{W}
\end{aligned}
$$

Then $V_{T_{1}}$ can be solved as:

$$
\begin{gathered}
V_{T_{1}}=\gamma V_{W}+(1-\gamma) V_{L}, \text { where } \\
\gamma=\frac{1-\left(1-P_{G}\left(x_{I}\right)\right) P_{G}\left(x_{E}\right)}{2-\left(1-P_{G}\left(x_{I}\right)\right) P_{G}\left(x_{E}\right)-P_{G}\left(x_{I}\right)\left(1-P_{G}\left(x_{B}\right)\right)} .
\end{gathered}
$$

As this is a zero-sum game, we have $V_{T_{2}}=(1-\gamma) V_{W}+\gamma V_{L}$. The optimal kicking strategy, $x_{I}$, satisfies the following first-order condition:

$$
P_{G}^{\prime}\left(x_{I}\right)\left[\left[P_{G}\left(x_{B}\right)-\left(1-P_{G}\left(x_{E}\right)\right)\right] V_{T_{2}}+\left(1-P_{G}\left(x_{B}\right)\right) V_{W}-P_{G}\left(x_{E}\right) V_{L}+U_{G}\right]+P_{O}^{\prime}\left(x_{I}\right) U_{O}=0 .
$$

Similarly, the optimal kicking strategies $x_{B}$ and $x_{E}$ are determined by the following conditions:

$$
\begin{aligned}
P_{G}^{\prime}\left(x_{B}\right)\left[V_{T_{1}}-V_{L}+U_{G}\right]+P_{O}^{\prime}\left(x_{B}\right) U_{O} & =0 \\
P_{G}^{\prime}\left(x_{E}\right)\left[V_{W}-V_{T_{1}}+U_{G}\right]+P_{O}^{\prime}\left(x_{E}\right) U_{O} & =0
\end{aligned}
$$

We are going to claim that all three kicking strategies are equivalent, i.e., $x_{I}=x_{B}=x_{E}$, which in turn implies that $V_{T_{1}}=V_{T_{2}}=\frac{V_{W}+V_{L}}{2}$ as $\gamma=\frac{1}{2}$, and order independence is established. First we compare $x_{I}$ and $x_{E}$. Define

$$
\Delta_{I E}=\left[P_{G}\left(x_{B}\right)-\left(1-P_{G}\left(x_{E}\right)\right)\right] V_{T_{2}}+\left(1-P_{G}\left(x_{B}\right)\right) V_{W}-P_{G}\left(x_{E}\right) V_{L}-\left(V_{W}-V_{T_{1}}\right)
$$

By comparing the first-order conditions of $x_{I}$ and $x_{E}$, we observe that

$$
\Delta_{I E} \gtreqless 0 \text { if and only if } x_{I} \gtreqless x_{E} \text {. }
$$

Substituting the equations of $V_{T_{2}}$ and $V_{T_{1}}$ into $\Delta_{I E}$ gives us

$$
\begin{aligned}
\Delta_{I E} & =V_{T_{1}}-V_{T_{2}}-P_{G}\left(x_{B}\right)\left(V_{W}-V_{T_{2}}\right)+P_{G}\left(x_{E}\right)\left(V_{T_{2}}-V_{L}\right) \\
& =\left[2 \gamma-1-P_{G}\left(x_{B}\right) \gamma+P_{G}\left(x_{E}\right)(1-\gamma)\right]\left(V_{W}-V_{L}\right) \\
& =\left[\left(2-P_{G}\left(x_{B}\right)-P_{G}\left(x_{E}\right)\right) \gamma-1+P_{G}\left(x_{E}\right)\right]\left(V_{W}-V_{L}\right) .
\end{aligned}
$$


Plugging in the expression of $\gamma$ and doing some simplifications, we have

$$
\Delta_{I E}=\frac{P_{G}\left(x_{I}\right)\left(1-P_{G}\left(x_{B}\right)\right)-P_{G}\left(x_{B}\right)\left(1-P_{G}\left(x_{E}\right)\right)}{2-\left(1-P_{G}\left(x_{I}\right)\right) P_{G}\left(x_{E}\right)-P_{G}\left(x_{I}\right)\left(1-P_{G}\left(x_{B}\right)\right)}\left(V_{W}-V_{L}\right)
$$

We can then conclude that $x_{I} \gtreqless x_{E}$ if and only if $x_{I} \gtreqless x_{B}$. Next we compare $x_{I}$ and $x_{B}$. Define

$$
\Delta_{I B}=\left[P_{G}\left(x_{B}\right)-\left(1-P_{G}\left(x_{E}\right)\right)\right] V_{T_{2}}+\left(1-P_{G}\left(x_{B}\right)\right) V_{W}-P_{G}\left(x_{E}\right) V_{L}-\left(V_{T_{1}}-V_{L}\right)
$$

By comparing the first-order conditions of $x_{I}$ and $x_{B}$, we observe that

$$
\Delta_{I B} \gtreqless 0 \text { if and only if } x_{I} \gtreqless x_{B} \text {. }
$$

By the same token, we can simplify $\Delta_{I B}$ as

$$
\Delta_{I B}=\frac{P_{G}\left(x_{E}\right)\left(1-P_{G}\left(x_{I}\right)\right)-P_{G}\left(x_{B}\right)\left(1-P_{G}\left(x_{E}\right)\right)}{2-\left(1-P_{G}\left(x_{I}\right)\right) P_{G}\left(x_{E}\right)-P_{G}\left(x_{I}\right)\left(1-P_{G}\left(x_{B}\right)\right)}\left(V_{W}-V_{L}\right)
$$

Therefore

$$
x_{I} \gtreqless x_{B} \text { if and only if } x_{E} \gtreqless x_{B} \text {. }
$$

Finally we compare $x_{E}$ and $x_{B}$. Define

$$
\Delta_{E B}=V_{W}-V_{T_{1}}-\left(V_{T_{1}}-V_{L}\right)
$$

$\Delta_{E B}$ can be simplified as

$$
\Delta_{E B}=\frac{P_{G}\left(x_{E}\right)\left(1-P_{G}\left(x_{I}\right)\right)-P_{G}\left(x_{I}\right)\left(1-P_{G}\left(x_{B}\right)\right)}{2-\left(1-P_{G}\left(x_{I}\right)\right) P_{G}\left(x_{E}\right)-P_{G}\left(x_{I}\right)\left(1-P_{G}\left(x_{B}\right)\right)}\left(V_{W}-V_{L}\right)
$$

Accordingly,

$$
x_{E} \gtreqless x_{B} \text { if and only if } x_{E} \gtreqless x_{I} \text {. }
$$

Combining all three observations (inequalities) above, we conclude that in a state-symmetric equilibrium we must have $x_{I}=x_{E}=x_{B}$.

Proof of Theorem 4. Take any mechanism $\phi$ and any order-independent mechanism $\varphi$. Construct a mechanism $\psi$ such that for a given Sudden-death Round $k$, for all $n<\ell<k$, kickingorder histories $h^{\ell-1}$, and feasible scores $g_{T_{1}}: g_{T_{2}}, \psi\left(h^{\ell-1} ; g_{T_{1}}: g_{T_{2}}\right)=\phi\left(h^{\ell-1} ; g_{T_{1}}: g_{T_{2}}\right)$ and for all $\ell \geq k$ and $\ell \leq n$, kicking-order histories $h^{\ell-1}$, and feasible scores $g_{T_{1}}: g_{T_{2}}, \psi\left(h^{\ell-1} ; g_{T_{1}}: g_{T_{2}}\right)=$ $\varphi\left(h^{\ell-1} ; g_{T_{1}}: g_{T_{2}}\right)$. Now in the Sudden-death Round $k$ and after, whenever the game reaches this round, the probability of winning is given as $\frac{1}{2}$ for each team. By backward induction, consider Round $k-1$. Consider the team that kicks second. Without loss of generality suppose it is Team 2 , and Team 1 goes first in Round $k-1$. We can reuse the same first-order conditions for both teams that we used in the proof of Theorem 2, setting

$$
V_{T_{1}}=V_{T_{2}}=\frac{V_{W}+V_{L}}{2}
$$


as the continuation value under the order-independent mechanism in Round $k$. Suppose $x$ is Team 1's kicker's optimal spot, $y_{E}$ is Team 2's kicker's optimal spot when they are still tied, and $y_{B}$ is Team 1's kicker's optimal spot when Team 1 is ahead (by one goal). Recall the first-order conditions through Equation 11 (or 3):

$$
\begin{gathered}
P_{G}^{\prime}(x)\left[P_{G}\left(y_{B}\right) V_{T_{1}}+\left(1-P_{G}\left(y_{B}\right)\right) V_{W}-P_{G}\left(y_{E}\right) V_{L}-\left(1-P_{G}\left(y_{E}\right)\right) V_{T_{1}}+U_{G}\right]+P_{O}^{\prime}(x) U_{O}=0 \\
P_{G}^{\prime}\left(y_{B}\right)\left[V_{T_{2}}-V_{L}+U_{G}\right]+P_{O}^{\prime}\left(y_{B}\right) U_{O}=0 \\
P_{G}^{\prime}\left(y_{E}\right)\left[V_{W}-V_{T_{2}}+U_{G}\right]+P_{O}^{\prime}\left(y_{E}\right) U_{O}=0
\end{gathered}
$$

We rewrite Team 2's kicker's first-order conditions plugging in $V_{T_{1}}=V_{T_{2}}$ :

$$
\begin{aligned}
& P_{G}^{\prime}\left(y_{B}\right)\left[\frac{V_{W}-V_{L}}{2}+U_{G}\right]+P_{O}^{\prime}\left(y_{B}\right) U_{O}=0 \\
& P_{G}^{\prime}\left(y_{E}\right)\left[\frac{V_{W}-V_{L}}{2}+U_{G}\right]+P_{O}^{\prime}\left(y_{E}\right) U_{O}=0
\end{aligned}
$$

The last two equations yield $y_{B}=y_{E}$ (each has a unique solution by assumptions). Given that Team 1's equation yields:

$$
P_{G}^{\prime}(x)\left[\frac{V_{W}-V_{L}}{2}+U_{G}\right]+P_{O}^{\prime}(x) U_{O}=0
$$

As Team 1 has the same first-order conditions as Team 2, we get $x=y_{B}=y_{E}$. So each team's winning probability is the same, $\frac{1}{2}$ in Round $k$, as well. The mechanism $\psi$ is order independent starting from Round $k$. We repeat this argument for each Sudden-death Round $\ell=k-2, k-$ $3, \ldots, n+1$ and obtain the desired result.

Proof of Theorem 6. The first-order conditions are given by Equations 29 and 30 for $y_{B}$ and $y_{E}$ in the proof of Theorem 2 , respectively. We get $y_{B}=y(\beta)$ and $y_{E}=y(1-\beta)$, since $f=P_{G}^{\prime} / P_{O}^{\prime}$ is an invertible differentiable decreasing function in the region $[0, \bar{x}]$ by assumption that $P_{O}$ is convex and increasing and $P_{G}$ is strictly concave and increasing in the interval $[0, \bar{x}]$. Thus, circularly, plugging in $y_{B}$ and $y_{E}$, we get Equation 27. Optimal spots $y_{B}$ and $x=y_{E}$ are multiple valued if and only if $\beta$ is multiple valued. $\beta=\frac{1}{2}$ always solves Equation 27 , and if $\beta=\alpha$ is a solution then $\beta=1-\alpha$ is also a solution. Thus, there is an odd number of solutions.

Sufficient Condition for Multiplicity:

First observe that $\Upsilon(0)<0$ and $\Upsilon\left(\frac{1}{2}\right)=0$. If $\left.\Upsilon^{\prime}(\beta)\right|_{\beta=\frac{1}{2}}<0$, then $\Upsilon\left(\frac{1}{2}-\epsilon\right)>0$ for $\epsilon$ sufficiently small. Combining this with the fact that $\Upsilon(0)<0$ and $\Upsilon(\beta)$ is continuous, we conclude that there exists some $\widehat{\beta} \in\left(0, \frac{1}{2}\right)$ such that $\Upsilon(\widehat{\beta})=0$ when $\left.\Upsilon^{\prime}(\beta)\right|_{\beta=\frac{1}{2}}<0$. Hence, $\Upsilon(\beta)$ has multiple solutions if $\left.\Upsilon^{\prime}(\beta)\right|_{\beta=\frac{1}{2}}<0$. In the following, we provide a sufficient condition for $\left.\Upsilon^{\prime}(\beta)\right|_{\beta=\frac{1}{2}}<0$.

We have

$$
\begin{aligned}
\left.\Upsilon^{\prime}(\beta)\right|_{\beta=\frac{1}{2}}= & 1-\left.\frac{\left\{\begin{array}{c}
{\left[P_{G}^{\prime}(y(1-\beta)) y^{\prime}(1-\beta)\right]\left[2-P_{G}(y(\beta))-P_{G}(y(1-\beta))\right]} \\
-\left[-P_{G}^{\prime}(y(\beta)) y^{\prime}(\beta)+P_{G}^{\prime}(y(1-\beta)) y^{\prime}(1-\beta)\right]\left[1-P_{G}(y(1-\beta))\right]
\end{array}\right.}{\left[2-P_{G}(y(\beta))-P_{G}(y(1-\beta))\right]^{2}}\right|_{\beta=\frac{1}{2}} \\
= & 1-\frac{P_{G}^{\prime}\left(y\left(\frac{1}{2}\right)\right)}{2\left(1-P_{G}\left(y\left(\frac{1}{2}\right)\right)\right)} y^{\prime}\left(\frac{1}{2}\right) .
\end{aligned}
$$


As $y(\beta)=f^{-1}\left(\frac{-U_{O}}{\left(V_{W}-V_{L}\right) \beta+U_{G}}\right)$ and $f(x)=P_{G}^{\prime}(x) / P_{O}^{\prime}(x), y^{\prime}\left(\frac{1}{2}\right)$ can be computed as:

$$
\begin{aligned}
y^{\prime}\left(\frac{1}{2}\right) & =\left.\frac{1}{f^{\prime}(y(\beta))} \frac{\left(V_{W}-V_{L}\right) U_{O}}{\left(\left(V_{W}-V_{L}\right) \beta+U_{G}\right)^{2}}\right|_{\beta=\frac{1}{2}} \\
& =\frac{\left(P_{O}^{\prime}\left(y\left(\frac{1}{2}\right)\right)\right)^{2}}{P_{G}^{\prime \prime}\left(y\left(\frac{1}{2}\right)\right) P_{O}^{\prime}\left(y\left(\frac{1}{2}\right)\right)-P_{O}^{\prime \prime}\left(y\left(\frac{1}{2}\right)\right) P_{G}^{\prime}\left(y\left(\frac{1}{2}\right)\right)} \cdot \frac{\left(V_{W}-V_{L}\right) U_{O}}{\left(\left(V_{W}-V_{L}\right) \frac{1}{2}+U_{G}\right)^{2}} .
\end{aligned}
$$

Also we have

$$
y\left(\frac{1}{2}\right)=f^{-1}\left(\frac{-U_{O}}{\left(V_{W}-V_{L}\right) \frac{1}{2}+U_{G}}\right) \Longrightarrow\left(V_{W}-V_{L}\right) \frac{1}{2}+U_{G}=-\frac{P_{O}^{\prime}\left(y\left(\frac{1}{2}\right)\right)}{P_{G}^{\prime}\left(y\left(\frac{1}{2}\right)\right)} U_{O} .
$$

Then

$$
\begin{aligned}
y^{\prime}\left(\frac{1}{2}\right) & =\frac{\left(P_{O}^{\prime}\left(y\left(\frac{1}{2}\right)\right)\right)^{2}}{P_{G}^{\prime \prime}\left(y\left(\frac{1}{2}\right)\right) P_{O}^{\prime}\left(y\left(\frac{1}{2}\right)\right)-P_{O}^{\prime \prime}\left(y\left(\frac{1}{2}\right)\right) P_{G}^{\prime}\left(y\left(\frac{1}{2}\right)\right)} \cdot \frac{V_{W}-V_{L}}{\left(V_{W}-V_{L}\right) \frac{1}{2}+U_{G}} \cdot \frac{-P_{G}^{\prime}\left(y\left(\frac{1}{2}\right)\right)}{P_{O}^{\prime}\left(y\left(\frac{1}{2}\right)\right)} \\
& =\frac{-P_{O}^{\prime}\left(y\left(\frac{1}{2}\right)\right) P_{G}^{\prime}\left(y\left(\frac{1}{2}\right)\right)}{P_{G}^{\prime \prime}\left(y\left(\frac{1}{2}\right)\right) P_{O}^{\prime}\left(y\left(\frac{1}{2}\right)\right)-P_{O}^{\prime \prime}\left(y\left(\frac{1}{2}\right)\right) P_{G}^{\prime}\left(y\left(\frac{1}{2}\right)\right)} \cdot \frac{V_{W}-V_{L}}{\left(V_{W}-V_{L}\right) \frac{1}{2}+U_{G}},
\end{aligned}
$$

and

$\left.\Upsilon^{\prime}(\beta)\right|_{\beta=\frac{1}{2}}=1-\frac{P_{G}^{\prime}\left(y\left(\frac{1}{2}\right)\right)}{2\left(1-P_{G}\left(y\left(\frac{1}{2}\right)\right)\right)} \cdot \frac{-P_{O}^{\prime}\left(y\left(\frac{1}{2}\right)\right) P_{G}^{\prime}\left(y\left(\frac{1}{2}\right)\right)}{P_{G}^{\prime \prime}\left(y\left(\frac{1}{2}\right)\right) P_{O}^{\prime}\left(y\left(\frac{1}{2}\right)\right)-P_{O}^{\prime \prime}\left(y\left(\frac{1}{2}\right)\right) P_{G}^{\prime}\left(y\left(\frac{1}{2}\right)\right)} \cdot \frac{V_{W}-V_{L}}{\left(V_{W}-V_{L}\right) \frac{1}{2}+U_{G}}$.

Therefore $\left.\Upsilon^{\prime}(\beta)\right|_{\beta=\frac{1}{2}}<0$ if and only if

$$
\begin{aligned}
& 1+\frac{2 U_{G}}{V_{W}-V_{L}}<\frac{-P_{G}^{\prime}\left(y\left(\frac{1}{2}\right)\right)}{1-P_{G}\left(y\left(\frac{1}{2}\right)\right)} \cdot \frac{P_{O}^{\prime}\left(y\left(\frac{1}{2}\right)\right) P_{G}^{\prime}\left(y\left(\frac{1}{2}\right)\right)}{P_{G}^{\prime \prime}\left(y\left(\frac{1}{2}\right)\right) P_{O}^{\prime}\left(y\left(\frac{1}{2}\right)\right)-P_{O}^{\prime \prime}\left(y\left(\frac{1}{2}\right)\right) P_{G}^{\prime}\left(y\left(\frac{1}{2}\right)\right)} \\
& \Longleftrightarrow \quad 1+\frac{2 U_{G}}{V_{W}-V_{L}}<\left.\frac{\left(\ln \left(1-P_{G}(x)\right)\right)^{\prime}}{(\ln f(x))^{\prime}}\right|_{x=y\left(\frac{1}{2}\right)} .
\end{aligned}
$$

\section{Field Evidence}

\section{D.1 Shootout Winning Percentages in Major Tournaments}


Figure 2: Empirical Evidence from Table 5.1 in Palacios-Huerta (2014) and Table 1 in Kocher, Lenz, and Sutter (2012): The winning proportions of first-kicking teams are given on the vertical axis while the numbers of shootouts in the considered championships are given on the horizontal axis. Euro int refers to combined proportion for all European international championships such as European Championship, Champions League, Cup Winners Cup, and UEFA Cup. Observe that as sample size increases (i.e., data points 50 or more) second-mover advantage disappears in major football data tournaments. While there is undisputed first-mover advantage in Spanish Cup, Euro int and English Cup display somewhat first-mover advantage, and German Cup displays neither first- nor second-mover advantage. We also thank Martin Kocher, Marc Lenz, and Matthias Sutter for providing us their data set.

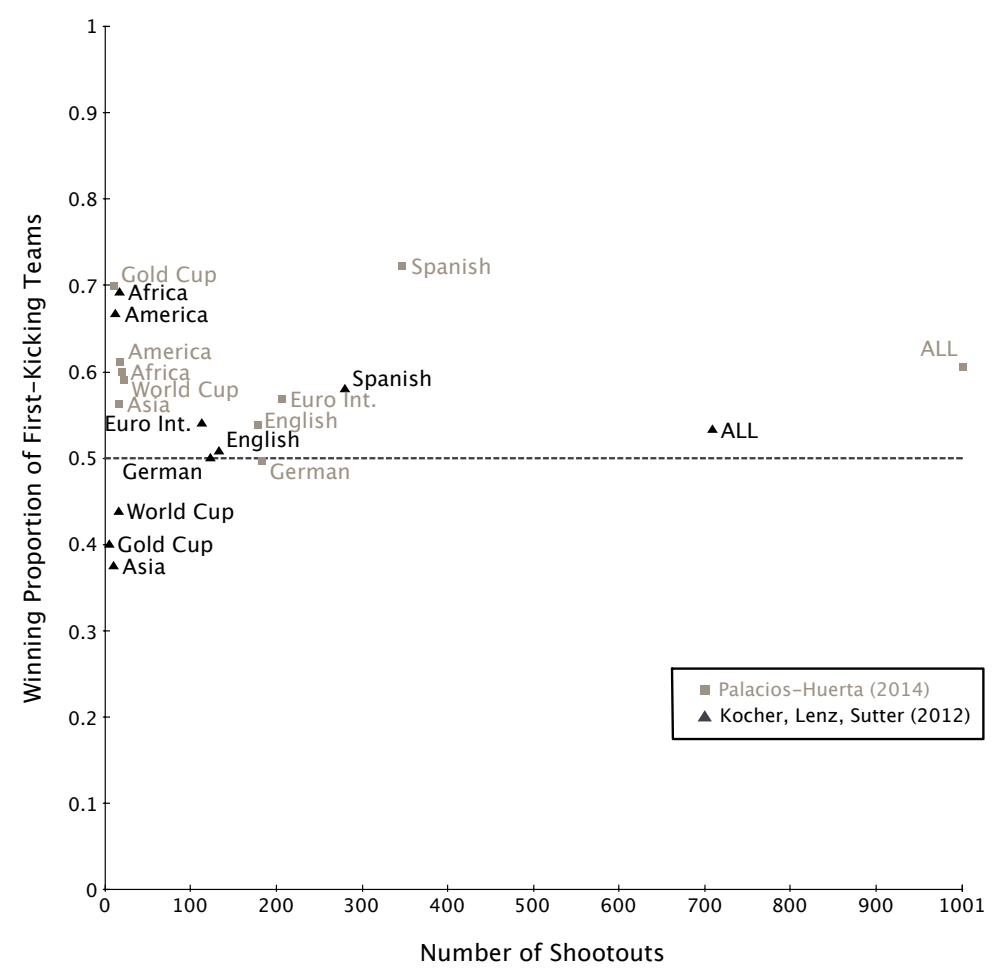




\section{D.2 ABBA vs ABAB in the Field}

Recently ABBA replaced ABAB at the U-17 Women's and Men's World Football Championships. The International Football Association Board (IFAB) decided to implement the ABBA sequence in various trials before eventually using it in Women's World Cup. In addition, the ABBA format for penalty shootouts is adopted in all English Football League (EFL) competitions in 2017-18, ${ }^{29}$ and recently in Dutch Cup in 2018-19, while the rest of the world still uses ABAB as of this writing. In dynamic individual contests too, we observe that the ABBA format being utilized. It is used in the U.S. presidential debate sequences. Similarly, FIDE, the governing body of chess, has recently changed the rules for the FIDE World Chess Championship and switched from ABAB to a generalized ABBA structure that reverses the sequence of players who play with white pieces at the half-way, i.e., ABABAB-BABABA. ${ }^{30}$ Traditionally, however, ABBA has always been used in tennis for serve order in tiebreak sets. ${ }^{31}$

The rationale behind experimenting with $\mathrm{ABBA}$ in football is the belief that ABBA will eliminate or at least alleviate a first-mover advantage if it exists. Indeed, using simple calculations assuming exogenous first-kick and second-kick success probabilities, Brams and Ismail (2018) and Echenique (2017) show that ABBA is less biased than ABAB. For example, Echenique performs calculations to argue that if the first-mover advantage ranges between $60 \%$ and $70 \%$ in the $\mathrm{ABAB}$ system, it should move to the $52-55 \%$ range under the ABBA system. We show in our paper that not only $\mathrm{ABAB}$ but $\mathrm{ABBA}$ is not order independent either, as it imposes an exogenous order sequence. However, ABBA is at least order independent in sudden-death rounds, while ABAB fails to satisfy it. Thus, problems associated with ABBA seem less severe. On the other hand, as ABBA has a unique state-symmetric equilibrium, it is also ex-ante fair as the team that goes first is determined with an initial even coin toss.

So far, since ABBA has been used in few instances and it did not replace ABAB in majority of the high-level competition, there is lack of sufficient data to suggest empirically that the problems associated with $\mathrm{ABAB}$ continue or are resolved under ABBA. It would be an interesting future research question to make controlled random trial experiments testing these mechanisms as well as the mechanisms that we propose.

\section{E Better Teams under Order Independent Mechanisms}

Uneven score symmetric mechanisms have another nice feature. Theorem 1 states that when two teams have the same kicking ability, they have equal winning probability. What if one team is better than the other? Suppose there is one player who has a better kicking ability than the rest of the

\footnotetext{
${ }^{29} \mathrm{EFL}$ manages the second-tier leagues and cups of English football.

${ }^{30}$ González-Díaz and Palacios-Huerta (2016) show that winning percentage in chess is in favor of the player drawing the white pieces in the first game with $60 \%-40 \%$ when ABAB was employed.

${ }^{31}$ Cohen-Zada et al. (2018) show that there is no first mover advantage with the ABBA format in tennis tiebreak serve sequences.
} 
players, i.e., the player has a higher $P_{G}(x)$ and a lower $P_{O}(x)$ for every $x \in[0,1]$. We formally define a better player as follows: Let $\left\{P_{G}, P_{O}\right\}$ represent all players' kicking ability except the better player, and $\left\{\widetilde{P}_{G}, \widetilde{P}_{O}\right\}$ represent the better player's kicking ability. We assume (a) $P_{G}(x)<\widetilde{P}_{G}(x)$ and $P_{O}(x)>\widetilde{P}_{O}(x)$, and $(\mathrm{b}) \frac{P_{G}^{\prime}(x)}{\widetilde{P}_{G}^{\prime}(x)}=\frac{P_{O}^{\prime}(x)}{\widetilde{P}_{O}^{\prime}(x)}$ for all $x \in[0,1]$. We show that the team with this better player - now named the better team - has a higher winning probability under uneven score symmetric mechanisms.

Theorem 7 [Uneven teams] Suppose a mechanism that is order independent in sudden-death rounds and uneven score symmetric in regular rounds is used in the shootout. Then a better team has a higher ex-ante chance of winning at the unique state-symmetric equilibrium of the shootout induced by this mechanism, if the better player is used strategically in the best kicking order possible by the better team.

Proof of Theorem 7. We show that by having the better player kick in Round 1, the better team has a higher chance of winning under an uneven score symmetric mechanism. Consider two subcases:

(i) When the better player is in Team 2. Since the better player is placed in Round 1, the second-round maximization problems remain unchanged. Following the proof of Theorem 1, we have $x_{2 A}=y_{2 A}>x_{2 B}=y_{2 B}$, and the last kicker's optimal kicking strategy is $\xi$. Next we study the second team's optimal kicking strategy in Round 1. When Team 1 does not score in Round 1, the value function for Team 2 is $^{32}$

$V_{T_{2}, P_{1}, E}=\widetilde{P}_{G}\left(y_{1 E}\right)\left[\phi\left(T_{1} ; 0: 1\right)\left(V_{W}+V_{L}-V_{T_{1}, P_{2}, B}\right)+\left(1-\phi\left(T_{1} ; 0: 1\right)\right) V_{T_{2}, P_{2}, A}\right]+\left(1-\widetilde{P}_{G}\left(y_{1 E}\right)\right) \frac{V_{W}+V_{L}}{2}$

where

$$
\begin{gathered}
V_{T_{1}, P_{2}, B}=P_{G}\left(x_{2 B}\right) P_{G}(\xi) V_{L}+P_{G}\left(x_{2 B}\right)\left(1-P_{G}(\xi)\right) \frac{V_{W}+V_{L}}{2}+\left(1-P_{G}\left(x_{2 B}\right)\right) V_{L} \\
=\frac{V_{W}+V_{L}}{2}-\left[1-P_{G}\left(x_{2 B}\right)\left(1-P_{G}(\xi)\right)\right] \frac{V_{W}-V_{L}}{2} \\
V_{T_{2}, P_{2}, A}=P_{G}\left(y_{2 A}\right) V_{W}+\left(1-P_{G}\left(y_{2 A}\right)\right)\left[\left(1-P_{G}(\xi)\right) V_{W}+P_{G}(\xi) \frac{V_{W}+V_{L}}{2}\right] \\
=\frac{V_{W}+V_{L}}{2}+\left[1-\left(1-P_{G}\left(y_{2 A}\right)\right) P_{G}(\xi)\right] \frac{V_{W}-V_{L}}{2}
\end{gathered}
$$

Therefore

$$
\begin{array}{r}
V_{T_{2}, P_{1}, E}=\frac{V_{W}+V_{L}}{2}+\widetilde{P}_{G}\left(y_{1 E}\right)\left\{\phi\left(T_{1} ; 0: 1\right)\left[1-P_{G}\left(x_{2 B}\right)\left(1-P_{G}(\xi)\right)\right]\right. \\
\left.+\left(1-\phi\left(T_{1} ; 0: 1\right)\right)\left[1-\left(1-P_{G}\left(y_{2 A}\right)\right) P_{G}(\xi)\right]\right\} \frac{V_{W}-V_{L}}{2}
\end{array}
$$

\footnotetext{
${ }^{32}$ Recall that kicking order $T_{1}$ in expression $\phi\left(T_{1} ; g_{T_{1}}: g_{T_{2}}\right)$ refers to the beginning of Round 2 when Team 1 kicked first in Round 1.
} 
The optimal kicking strategy, $y_{1 E}$, satisfies the following first-order condition:

$$
\begin{gathered}
\widetilde{P}_{G}^{\prime}\left(y_{1 E}\right)\left[\alpha_{1} \frac{V_{W}-V_{L}}{2}+U_{G}\right]+\widetilde{P}_{O}^{\prime}\left(y_{1 E}\right) U_{O}=0, \text { where } \\
\alpha_{1}=\phi\left(T_{1} ; 0: 1\right)\left[1-P_{G}\left(x_{2 B}\right)\left(1-P_{G}(\xi)\right)\right]+\left(1-\phi\left(T_{1} ; 0: 1\right)\right)\left[1-\left(1-P_{G}\left(y_{2 A}\right)\right) P_{G}(\xi)\right] .
\end{gathered}
$$

When Team 1 scores in Round 1, the value function for Team 2 is

$V_{T_{2}, P_{1}, B}=\widetilde{P}_{G}\left(y_{1 B}\right) \frac{V_{W}+V_{L}}{2}+\left(1-\widetilde{P}_{G}\left(y_{1 B}\right)\right)\left[\left(1-\phi\left(T_{1} ; 1: 0\right)\right) V_{T_{2}, P_{2}, B}+\phi\left(T_{1} ; 1: 0\right)\left(V_{W}+V_{L}-V_{T_{1}, P_{2}, A}\right)\right]$ where

$$
\begin{gathered}
V_{T_{2}, P_{2}, B}=P_{G}\left(y_{2 B}\right) P_{G}(\xi) V_{L}+P_{G}\left(y_{2 B}\right)\left(1-P_{G}(\xi)\right) \frac{V_{W}+V_{L}}{2}+\left(1-P_{G}\left(y_{2 B}\right)\right) V_{L} \\
=\frac{V_{W}+V_{L}}{2}-\left[1-P_{G}\left(y_{2 B}\right)\left(1-P_{G}(\xi)\right)\right] \frac{V_{W}-V_{L}}{2} \\
V_{T_{1}, P_{2}, A}=P_{G}\left(x_{2 A}\right) V_{W}+\left(1-P_{G}\left(x_{2 A}\right)\right)\left[\left(1-P_{G}(\xi)\right) V_{W}+P_{G}(\xi) \frac{V_{W}+V_{L}}{2}\right] \\
=\frac{V_{W}+V_{L}}{2}+\left[1-\left(1-P_{G}\left(x_{2 A}\right)\right) P_{G}(\xi)\right] \frac{V_{W}-V_{L}}{2}
\end{gathered}
$$

We substitute the equations of $V_{T_{2}, P_{2}, B}$ and $V_{T_{1}, P_{2}, A}$ into $V_{T_{2}, P_{1}, B}$ as follows:

$$
\begin{aligned}
& V_{T_{2}, P_{1}, B}=\frac{V_{W}+V_{L}}{2}-\left(1-\widetilde{P}_{G}\left(y_{1 B}\right)\right)\left[\left(1-\phi\left(T_{1} ; 1: 0\right)\right)\left[1-P_{G}\left(y_{2 B}\right)\left(1-P_{G}(\xi)\right)\right]\right. \\
& \left.+\phi\left(T_{1} ; 1: 0\right)\left[1-\left(1-P_{G}\left(x_{2 A}\right)\right) P_{G}(\xi)\right]\right] \frac{V_{W}-V_{L}}{2}
\end{aligned}
$$

The optimal kicking strategy, $y_{1 B}$, satisfies the following first-order condition:

$$
\begin{aligned}
& \widetilde{P}_{G}^{\prime}\left(y_{1 B}\right)\left[\left[\left[\left(1-\phi\left(T_{1} ; 1: 0\right)\right)\left[1-P_{G}\left(y_{2 B}\right)\left(1-P_{G}(\xi)\right)\right]\right.\right.\right. \\
& \left.\left.\quad+\phi\left(T_{1} ; 1: 0\right)\left[1-\left(1-P_{G}\left(x_{2 A}\right)\right) P_{G}(\xi)\right]\right] \frac{V_{W}-V_{L}}{2}+U_{G}\right]+\widetilde{P}_{O}^{\prime}\left(y_{1 B}\right) U_{O}=0
\end{aligned}
$$

Given that $y_{2 B}=x_{2 B}$ and $x_{2 A}=y_{2 A}$, the first-order condition can be rewritten as

$$
\begin{gathered}
P_{G}^{\prime}\left(y_{1 B}\right)\left[\alpha_{2} \frac{V_{W}-V_{L}}{2}+U_{G}\right]+P_{O}^{\prime}\left(y_{1 B}\right) U_{O}=0, \text { where } \\
\alpha_{2}=\left(1-\phi\left(T_{1} ; 1: 0\right)\right)\left[1-P_{G}\left(x_{2 B}\right)\left(1-P_{G}(\xi)\right)\right]+\phi\left(T_{1} ; 1: 0\right)\left[1-\left(1-P_{G}\left(y_{2 A}\right)\right) P_{G}(\xi)\right] .
\end{gathered}
$$

Under an order-independent mechanism, $\phi\left(T_{1} ; 0: 1\right)+\phi\left(T_{1} ; 1: 0\right)=1$, and we have $\alpha_{1}=\alpha_{2}$. Accordingly, $y_{1 E}=y_{1 B}$. Finally, we solve for Team 1's optimal kicking strategy in Round 1 . The value function for Team 1 is

$$
\begin{aligned}
V_{T_{1}} & =P_{G}\left(x_{1}\right)\left[V_{W}+V_{L}-V_{T_{2}, P_{1}, B}\right]+\left(1-P_{G}\left(x_{1}\right)\right)\left[V_{W}+V_{L}-V_{T_{2}, P_{1}, E}\right] \\
& =V_{W}+V_{L}-P_{G}\left(x_{1}\right) V_{T_{2}, P_{1}, B}-\left(1-P_{G}\left(x_{1}\right)\right) V_{T_{2}, P_{1}, E} \\
& =\frac{V_{W}+V_{L}}{2}+\left[P_{G}\left(x_{1}\right)\left(1-\widetilde{P}_{G}\left(y_{1 B}\right)\right) \alpha_{2}-\left(1-P_{G}\left(x_{1}\right)\right) \widetilde{P}_{G}\left(y_{1 E}\right) \alpha_{1}\right] \frac{V_{W}-V_{L}}{2}
\end{aligned}
$$


The optimal kicking strategy, $x_{1}$, satisfies the following first-order condition:

$$
\begin{aligned}
& P_{G}^{\prime}\left(x_{1}\right)\left[\left[\left(1-\widetilde{P}_{G}\left(y_{1 B}\right)\right) \alpha_{2}\right.\right.\left.\left.+\widetilde{P}_{G}\left(y_{1 E}\right) \alpha_{1}\right] \frac{V_{W}-V_{L}}{2}+U_{G}\right]+P_{O}^{\prime}\left(x_{1}\right) U_{O}=0 \\
& \Longrightarrow P_{G}^{\prime}\left(x_{1}\right)\left[\alpha_{1} \frac{V_{W}-V_{L}}{2}+U_{G}\right]+P_{O}^{\prime}\left(x_{1}\right) U_{O}=0
\end{aligned}
$$

Therefore $x_{1}=y_{1 E}=y_{1 B}$, and

$$
V_{T_{1}}=\frac{V_{W}+V_{L}}{2}+\left[P_{G}\left(x_{1}\right)\left(1-\widetilde{P}_{G}\left(x_{1}\right)\right)-\left(1-P_{G}\left(x_{1}\right)\right) \widetilde{P}_{G}\left(x_{1}\right)\right] \alpha_{1} \frac{V_{W}-V_{L}}{2}<\frac{V_{W}+V_{L}}{2} .
$$

Hence Team 2 has a higher chance of winning.

(ii) When the better player is in Team 1. Following the same procedure in (i), we conclude $x_{1}=y_{1 E}=y_{1 B}$. But now $V_{T_{1}}$ becomes

$$
\begin{aligned}
V_{T_{1}} & =\widetilde{P}_{G}\left(x_{1}\right)\left[V_{W}+V_{L}-V_{T_{2}, P_{1}, B}\right]+\left(1-\widetilde{P}_{G}\left(x_{1}\right)\right)\left[V_{W}+V_{L}-V_{T_{2}, P_{1}, E}\right] \\
& =V_{W}+V_{L}-\widetilde{P}_{G}\left(x_{1}\right) V_{T_{2}, P_{1}, B}-\left(1-\widetilde{P}_{G}\left(x_{1}\right)\right) V_{T_{2}, P_{1}, E} \\
& =\frac{V_{W}+V_{L}}{2}+\left[\widetilde{P}_{G}\left(x_{1}\right)\left(1-P_{G}\left(x_{1}\right)\right)-\left(1-\widetilde{P}_{G}\left(x_{1}\right)\right) P_{G}\left(x_{1}\right)\right] \alpha_{1} \frac{V_{W}-V_{L}}{2}>\frac{V_{W}+V_{L}}{2} .
\end{aligned}
$$

Again, the team with a better player has a higher chance of winning.

Therefore, order-independent mechanisms satisfy the second part of the Aristotelean Justice criterion according to the definition of better/worse teams above. Unfortunately, we cannot make any more generalizations for other types of asymmetries between abilities of teams.

\section{F Practical Considerations}

Order independence is capable of ruling out almost all exogenous mechanisms in regular rounds, including $\mathrm{ABAB}$ and $\mathrm{ABBA}$. In terms of endogenous mechanisms, however, order independence does not pose as much of a restriction. One needs further desirable properties to help refine the set of order-independent mechanisms. We will next define additional criteria to provide concrete practical advice in that regard. We can talk about efficiency or inefficiency of an attempt, to the extent it maximizes the effort levels of kickers. In other economic contests, efficiency could be even more important. Among order-independent mechanisms, it turns out that behind first and ahead first are the two extremes in terms of inducing kickers' efforts in Round 1:

Proposition 3 Behind-first (ahead-first) mechanisms maximize (minimize) Round 1 efforts of all kickers among all order-independent mechanisms at the state-symmetric equilibrium.

Proof of Proposition 3. From the proof of Theorem 1, we observe that any order-independent mechanism $\phi$ must satisfy the condition $\phi\left(T_{1} ; 0: 1\right)+\phi\left(T_{1} ; 1: 0\right)=1 .{ }^{33}$ Moreover, under this

\footnotetext{
${ }^{33}$ Recall that kicking order $T_{1}$ in expression $\phi\left(T_{1} ; g_{T_{2}}: g_{T_{2}}\right)$ refers to the beginning of Round 2 when Team 1 kicked first in Round 1.
} 
condition, the three optimal kicking strategies in Round 1 are the same: $x_{1}=y_{1 E}=y_{1 B}$ (see Corollary 1) and they are determined by the following first-order condition:

$$
\begin{gathered}
P_{G}^{\prime}\left(x_{1}\right)\left[\alpha_{1} \frac{V_{W}-V_{L}}{2}+U_{G}\right]+P_{O}^{\prime}\left(x_{1}\right) U_{O}=0, \text { where } \\
\alpha_{1}=\phi\left(T_{1} ; 1: 0\right)\left[1-\left(1-P_{G}\left(y_{2 A}\right)\right) P_{G}(\xi)\right]+\left(1-\phi\left(T_{1} ; 1: 0\right)\right)\left[1-P_{G}\left(x_{2 B}\right)\left(1-P_{G}(\xi)\right)\right] .
\end{gathered}
$$

Hence the higher the value of $\alpha_{1}$, the higher $x_{1}$. As $x_{2 B}<\xi$, which is Round 2 second kicking team's intended spot, and $y_{2 A}<\xi$, we obtain $1-P_{G}\left(x_{2 B}\right)\left(1-P_{G}(\xi)\right)>1-\left(1-P_{G}\left(y_{2 A}\right)\right) P_{G}(\xi)$. Therefore maximum $x_{1}$ is achieved in an order-independent mechanism when $\phi\left(T_{1} ; 1: 0\right)=0$, i.e., when $\phi$ is a behind-first mechanism. On the other hand, minimum $x_{1}$ is achieved when $\phi\left(T_{1} ; 1: 0\right)=1$, i.e., when $\phi$ is an ahead-first mechanism.

The intuition behind this result can be summarized as follows for behind first (ahead first is symmetric). First we summarize the incentives facing Round 2 kickers. In Round 2, kicking first is not good at all for higher goal efforts: the first-kicking team's player (if his team is either behind or ahead) will always exert less effort than he would in the case when he kicks second in Round 2. This is true because his marginal contribution will be less in the first case, as the other team's kicker who will go second - can always miss or offset the first kicker's failure. So he has higher incentives to shirk when he kicks first. Now, we turn our attention to Round 1 kickers' marginal contributions under both mechanisms. First, observe that both teams' kickers under any uneven score symmetric mechanism exert the same effort in Round 1, by Corollary 1. Therefore, understanding the firstkicking team player's incentives is sufficient to draw the difference between the two mechanisms regardless of the kicking order or score during Round 1. A Round 1 kicker, if he does not exert high effort under behind first, may cause his team to fall behind with higher probability. This causes his teammate to shirk more, when he goes first, and the other team's second player to exert higher effort, when he goes second in Round 2. On the other hand, under ahead first, the Round 1 kicker's incentives are exactly the opposite! If he does not exert high effort in Round 1, his team may fall behind with higher probability, but his teammate will exert relatively higher effort under ahead first by going second in Round 2 (with respect to behind first) and the other team's second kicker will exert less effort in Round 2 (with respect to behind first). Hence, Round 1 kicker's possible failure can still be salvaged with higher probability under ahead first. So he shirks under ahead first vis-avis behind first. Therefore, behind first dominates any random (i.e., convex combination of ahead first and behind first) and ahead-first mechanisms among all uneven score symmetric mechanisms. On the other hand, observe that ahead first and behind first cannot be compared with each other in Round 2 whenever the score is not tied: in ahead first when Team 1 is ahead, Team 1 kicks first while in behind first, it kicks second under the same scenario. So there are no two comparable information sets that are reached with positive probability under both mechanisms in Round 2. When the score is tied however, all uneven score symmetric mechanisms lead to the same goal efforts and are equivalent in Round 2. Thus, round by round we are not able to establish an effort ranking among different order-independent shootout mechanisms. Nevertheless, we can still obtain 
a partial ranking based on the expected number of attempts. Maximizing the expected number of attempts can be desirable from a social perspective, as well as a team perspective, if the attempt cost is negligible or negative. Spectators may wish to see more scoring attempts. Recall that we had defined two particular cases of shootouts earlier. In easy shootouts, the goal probability of each kick is greater than $50 \%$ for each intended spot, as in football, while in difficult shootouts, the goal probability of each kick is less than $50 \%$ for each intended spot, as in hockey. Then we have the following result as a proposition:

Proposition 4 An order-independent mechanism maximizes the expected number of attempts taken by both teams if and only if

- it is behind first in an easy shootout, and

- it is ahead first in a difficult shootout.

Proof of Proposition 4. When a tie occurs at the end of Round 1, both teams take a shot with probability 1 in Round 2. So an attempt is not taken only if the game is not tied at the end of Round 1. As both team players always exert the same effort $\xi_{1}$ in an order-independent mechanism at all state-symmetric equilibria in Round 1 (see the Corollary 1), the probability of one team being ahead at the beginning of Round 2 is $2 P_{G}\left(\xi_{1}\right)\left(1-P_{G}\left(\xi_{1}\right)\right)$. Consider any uneven score symmetric mechanism in which the ahead team moves first with probability $\alpha \in[0,1]$ in Round 2 . An attempt from the second-moving team in Round 2 is not made if and only if the ahead team moves first and scores or the behind team moves first and misses. This happens with a probability $2 P_{G}\left(\xi_{1}\right)\left(1-P_{G}\left(\xi_{1}\right)\right)\left[\alpha P_{G}\left(\xi_{2 A}\right)+(1-\alpha)\left(1-P_{G}\left(\xi_{2 B}\right)\right)\right]$, where $\xi_{1}$ is the Round 1 effort level of this shootout and $\xi_{2 B}, \xi_{2 A}$ are the Round 2 effort levels at state-symmetric equilibrium for the firstmoving team. For an easy shootout, we have $P_{G}(x)>\frac{1}{2}$ for all $x \in[0, \bar{x}]$. Given that the optimal first-period effort $\xi_{1}<\bar{x}, 2 P_{G}\left(\xi_{1}\right)\left(1-P_{G}\left(\xi_{1}\right)\right)$ is decreasing in $\xi_{1}$. Since behind first maximizes $\xi_{1}$ among all order-independent shootouts by Proposition 3, $2 P_{G}\left(\xi_{1}\right)\left(1-P_{G}\left(\xi_{1}\right)\right)$ is minimized under behind first. Moreover, in an easy shootout $1-P_{G}\left(\xi_{2 B}\right)<\frac{1}{2}<P_{G}\left(\xi_{2 A}\right)$. Hence, the probability of not making an attempt is minimized for behind first among all order-independent easy shootouts. For a difficult shootout, we have $P_{G}(x)<\frac{1}{2}$ for all $x \in[0, \bar{x}]$. Given that the optimal first-period effort $\xi_{1}<\bar{x}, 2 P_{G}\left(\xi_{1}\right)\left(1-P_{G}\left(\xi_{1}\right)\right)$ is increasing in $\xi_{1}$. Since ahead first minimizes $\xi_{1}$ among all order-independent shootouts by Proposition $3,2 P_{G}\left(\xi_{1}\right)\left(1-P_{G}\left(\xi_{1}\right)\right)$ is minimized under ahead first. Moreover, in a difficult shootout $1-P_{G}\left(\xi_{2 B}\right)>\frac{1}{2}>P_{G}\left(\xi_{2 A}\right)$. Hence, the probability of not making an attempt is minimized for ahead first among all order-independent easy shootouts.

The intuition for this result is as follows for easy shootouts: In Round 1, all teams take penalties. In Round 2 however, both teams take penalties for sure if and only if the score is tied after Round 1. It turns out that this occurs with the most probability in a behind-first shootout (see Proposition 3). Moreover, when the score is not tied after Round 1, the second-moving team in Round 2 
does not take a kick if the ahead team moves first and scores or the behind team moves first and misses. Given that in an easy shootout the probability of scoring is higher than the probability of missing, this probability is minimized under behind first. Hence, overall, behind first maximizes the expected number of attempts among all order-independent mechanisms. The intuition is reversed for difficult shootouts. Although behind-first mechanisms have nice features when the score is uneven, as mentioned before they are silent on how to define the kicking order when the score is tied. Order independence in regular rounds, by our characterization in Theorem 1, is also mute on this issue, but reversing the kicking order is a sure way of establishing order independence in sudden-death rounds (Theorem 3). ABBA, which is not order independent in regular rounds since it does not satisfy uneven score symmetry, does possess a nice property: When the score is tied in most crucial rounds, i.e., in sudden-death rounds, it gives both teams an equality of opportunity of kicking first. Clearly, such an equality-of-opportunity property is nowhere more important than in sudden-death rounds in which the score must be tied before every round. We would like to preserve the equality-of-opportunity feature of this mechanism, especially in sudden-death rounds. The behind-first (ahead-first) mechanism defined below has this feature.

The alternating-order behind-first (ahead-first) mechanism: The team that is behind (ahead) in score after any Round $r$ kicks first in Round $r+1$. If the score is tied after Round $r$, then the team that kicked second in Round $r$ kicks first in Round $r+1 .{ }^{34}$

Besides its simplicity, this mechanism possesses several nice features. We will start with suddendeath equality of opportunity. This property would emerge naturally since a simple but strong case could be easily made against the same team kicking multiple times in a row in those rounds in a lop-sided fashion:

Sudden-death equality of opportunity: Whenever the shootout ends after the Sudden-death Round $n+r$ with $r$ even, each team will have kicked first exactly $r / 2$ times in the sudden-death rounds.

Then we have the following corollary:

Corollary 2 The alternating-order behind-first (ahead-first) mechanism satisfies sudden-death equality of opportunity.

Another justification of alternating-order behind first and ahead first is as follows: Eclectic mechanisms could be confusing for players, coaches, referees, and fans. One can combine an orderindependent mechanism in regular rounds with another order-independent mechanism in suddendeath rounds in an eclectic fashion to come up with an overall order-independent mechanism. For example, consider the following mechanism in regular rounds coupled with the alternating-order

\footnotetext{
${ }^{34}$ We are agnostic about how Round 1 order is determined in the definition of the mechanism. It can be determined in any manner. However, in practice we suggest it be determined by an even coin toss as in ABAB.
} 
mechanism in sudden-death rounds: Team 1 kicks first in Round $r$ as long as the score is tied or Team 1 is behind in Round $r-1$; once Team 2 falls behind after some Round $r^{\prime}>r$, Team 2 kicks first until Team 1 falls behind in score after some Round $r^{\prime \prime}>r^{\prime}$, after which Team 1 kicks first. One can improve on such a patchy mechanism by requiring that such an eclecticism should be eliminated. We will introduce two properties such that the latter uses the former in its definition to formalize this intuition of simplicity. Before introducing the first property, we formally introduce how an order pattern can be recognized in a mechanism:

A finite machine representation of a mechanism is a triple $(Q, A, t)$ such that

- $Q$ is a finite set of (machine) states such that state $q=\left(T_{k}\right)_{w} \in Q$ denotes that Team $k$ taking the first penalty shot in the round associated with this state and $w$ is just an index number. Thus, $Q$ can be partitioned into two as $Q_{T_{1}}=\left\{\left(T_{1}\right)_{1}, \ldots,\left(T_{1}\right)_{w_{1}}\right\}$ and $Q_{T_{2}}=$ $\left\{\left(T_{2}, \ldots,\left(T_{2}\right)_{w_{2}}\right\}\right.$ for some $w_{1}$ and $w_{2}$ as the sets of states in which Team 1 and Team 2 kick first, respectively.

- $A=\left\{\left(g_{1}: g_{2}\right)\right\}$ is the set of possible scores.

- $t: Q \cup\{\emptyset\} \times A \times Q \rightarrow[0,1]$ is a state transition probability function such that $\sum_{q^{\prime} \in Q} t\left(q,\left(g_{1}: g_{2}\right), q^{\prime}\right)=1$ for all $q \in Q \cup\{\emptyset\}$ and $\left(g_{1}: g_{2}\right) \in A$. Here, $t\left(q,\left(g_{1}: g_{2}\right), q^{\prime}\right)$ is the probability of moving from state $q$ to state $q^{\prime}$ when after round associated with $q$ is played and the score is $g_{1}: g_{2}$ just before $q^{\prime}$ and after $q$.

We refer to null state $\emptyset$, as the start of the shootout. In this representation, we envision that each machine state is associated with a round of penalty kicks taken by each team consecutively. However, as round numbers proceed, the game will have to come back to some previous machine state, as the set of states is finite whereas a game can last arbitrarily long in theory. A mechanism $\phi$ is said to have finite machine representation $(Q, A, t)$, if $(1) t\left(\emptyset,(0: 0),\left(T_{1}\right)_{1}\right)=\phi(\emptyset, 0: 0)$ and $t\left(\emptyset,(0: 0),\left(T_{2}\right)_{1}\right)=1-\phi(\emptyset, 0: 0)$; and (2) recursively, for any kicking-order history $h^{r-1}$ at the beginning of Round $r$, and feasible score $g_{T_{1}}: g_{T_{2}}$ at the beginning of Round $r$, if the associated machine state with round $r-1$ was $q \in Q$, then we have $t\left(q,\left(g_{T_{1}}: g_{T_{2}}\right),\left(T_{1}\right)_{w}\right)=\phi\left(h^{r-1}, g_{T_{1}}: g_{T_{2}}\right)$ for some state $\left(T_{1}\right)_{w} \in Q_{T_{1}}$ and $t\left(q,\left(g_{T_{1}}: g_{T_{2}}\right),\left(T_{2}\right)_{w}\right)=1-\phi\left(h^{r-1}, g_{T_{1}}: g_{T_{2}}\right)$ for some state $\left(T_{2}\right)_{w} \in Q_{T_{2}}$; and once a transition occurs to a state $q^{\prime}$ from $q$, ex post we refer to $q^{\prime}$ as the machine state associated with round $r$. Note that a machine representation does not specify when the shootout game ends, as no round information is kept in the machine representation. It only keeps track of how transitions are made between different kicking orders in a well-defined pattern. We are now ready to introduce our next property:

A mechanism is stationary if it has a finite machine representation $(Q, A, t)$ such that for all states $q_{i} \in Q \cup\{\emptyset\}$ and $q_{j} \in Q, t\left(q_{i},\left(g_{T_{1}}: g_{T_{2}}\right), q_{j}\right)=t\left(q_{i},\left(g_{T_{1}}^{\prime}: g_{T_{2}}^{\prime}\right), q_{j}\right)$ for all scores such that $g_{T_{1}}-g_{T_{2}}=g_{T_{1}}^{\prime}-g_{T_{2}}^{\prime}$.

Thus, stationarity implies that state transitions are made in the same manner whenever score 
differences are the same. For example, the alternating-order behind-first mechanism has this type of a representation as shown in Figure 3. We state the following proposition whose proof is given

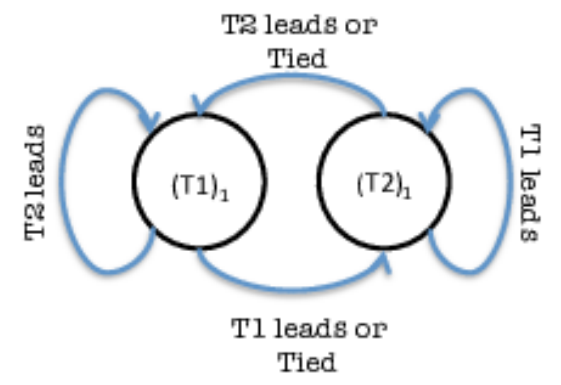

Figure 3: The state transition representation for the alternating-order behind-first mechanism. Transitions from the start of the shootout are omitted for simplicity. In general one of the two states in the figure will be chosen randomly with an unbiased lottery. Alternating-order ahead first's representation is symmetrically defined.

in the figure for behind first:

Proposition 5 The alternating-order behind-first (ahead-first) mechanism is stationary.

Machine representations can be used to measure the complexity of an algorithm. ${ }^{35}$ However, very complicated mechanisms can also be stationary. ${ }^{36}$ On the other hand, if we would like to have a chance of both teams kicking first in at least one round, we need at least two states, one Team-1-kicking-first state and one Team-2-kicking-first state. Thus, $|Q|=2$ is the minimum we can hope for in a reasonable mechanism. ${ }^{37}$ Our alternating-order behind-first mechanism also has this property (see Figure 3). We formalize this property as follows:

A mechanism is simple if it has a stationary machine representation with only two states such that in one state Team 1 kicks first and in the other Team 2 kicks first. An important motivation for simplicity stems from the FIFA football rules. These rules state that a rule violation by the referees

\footnotetext{
${ }^{35}$ For example, in game theory, they are used to represent the recall requirement needed for implementing a repeated game strategy (see Rubinstein, 1998, for an excellent survey).

${ }^{36}$ Consider a modified Prouhet-Thue-Morse behind-first mechanism. First we define the fractal Prouhet-ThueMorse mechanism (see Palacios-Huerta, 2014): The kicking order proceeds in an exogenous manner as follows: ABBABAAB..., i.e., the order sequence since the beginning of the shootout reverses after $2^{k}$ rounds for each $k=$ $1,2, \ldots$. We define the following modified Prouhet-Thue-Morse behind-first mechanism: If one team is behind, it kicks first; otherwise, at even scores the first-kicking team follows the sequence ABBABAAB; then this sequence reverses starting with Team 2 and keeps reversing until the shootout ends. Any behind-first mechanism compatible with a Prouhet-Thue-Morse order is stationary, and the simplest stationary machine representation of such a mechanism cannot have fewer than $|Q|=16$ states. On the other hand, the truly fractal Prouhet-Thue-Morse sequence is not stationary.

${ }^{37}$ Indeed, ABAB has $|Q|=2$, as according to the initial coin toss, either team can go first. However, it is not order independent. The random-order mechanism has also $|Q|=2$ and is order independent, however, does not maximize the expected number of attempts.
} 
during a game necessitates replay of the game. Shootout mechanisms that satisfy the simplicity property will make the process easier to administer for the referees and will make the process less prone to rule violations. We see simplicity as a vital requirement of a real-life shootout mechanism. The current mechanism satisfies simplicity but none of the other properties we have introduced in this paper. We formalize the simplicity of the alternating-order behind first (ahead first) with the following proposition. We gave its proof earlier through Figure 3:

Proposition 6 The alternating-order behind-first (ahead-first) mechanism is simple.

We state the main result of this appendix as follows (which was stated as Theorem 5 in Discussion section of the main text).

Theorem 8 In an easy shootout, alternating order behind first is the unique order-independent mechanism that maximizes the expected number of attempts and satisfies simplicity and suddendeath equality of opportunity. On the other hand, in a difficult shootout, alternating order ahead first is the unique order-independent mechanism that maximizes the expected number of attempts and satisfies simplicity and sudden-death equality of opportunity.

Proof of Theorem 8. Observe that the mechanisms that satisfy the properties should be behind-first, since behind-first mechanisms are the only ones that satisfy order independence and maximizing expected number of attempts (by Proposition 4). The mechanisms that satisfy the sudden-death equality of opportunity (SDEO from now on) have to have each team kicking first in every two sudden-death rounds exactly once. Hence, the only kicking order that is simple and SDEO in the sudden-death rounds is alternating-order. Stationarity (as implied by simplicity) implies that the order of kicking switches when the score stays even between two rounds - i.e., if the state was reached after a tie in score, the order switches after this state if the tied score continues. But this does not imply how the kicking order changes if we transition to a tied score from an uneven score. Simplicity implies that we have two states as $Q=\left\{\left(T_{1}\right)_{1},\left(T_{2}\right)_{1}\right\}$. Thus, we need to use the same states of sudden-death rounds also in the regular rounds. Hence, as kicking order switches when the score is tied, i.e. we transition from $\left(T_{1}\right)_{1}$ to $\left(T_{2}\right)_{1}$ or the other way around in the sudden-death rounds, we should do the same in the regular rounds as well. Thus, whenever a round ends with a tied score, we should reverse the kicking order. We end up with the unique machine representation in Figure 3, i.e. with the alternating-order behind-first.

We next demonstrate the independence of properties in Theorem 8: An order-independent mechanism that satisfies all properties but violates the maximization of expected number of attempts is the alternating-order ahead-first (behind-first) mechanism for easy (difficult) shootouts. An orderindependent mechanism that satisfies all properties but the sudden-death equality of opportunity is a behind-first (an ahead-first) mechanism for easy (difficult) shootouts, which randomly determines with an even lottery who goes first when the score is tied. An order-independent mechanism 
that satisfies all properties but is not simple is a Prouhet-Thue-Morse behind-first (ahead-first) mechanism for easy (difficult) shootouts.

Finally, an interesting and relevant question is whether the behind-first feature has been used in real life. Perhaps it is nowhere more blatant and effectively at work than in the rules of petanque (a.k.a. boules or bocce), which was invented in ancient times by the Greeks, later modified by Romans, and is now popular in various parts of the world including France and Italy - and currently expanding. ${ }^{38}$ In this game, the goal is to throw metal or wooden balls as close as possible to a small special wooden target, while standing inside a small starting circle. The rules are as follows: A player from the team that threw (and established) the target also throws the first ball. Then a player from the other team throws the second ball. The team with the ball that is closest to the target is said to have the point or be winning and other team is losing. Then the losing team gets to throw the next ball. ${ }^{39}$ Thus, in essence, just like our behind-first mechanisms, petanque too intends to give the losing team a chance to recover. Further, if the two balls closest to the target are from opposing teams and equidistant, teams play alternately until one team becomes the winning team and the other one the losing team. ${ }^{40}$

\section{G Order Independent Mechanisms with Three Regular Rounds}

Let us define $V_{i, j, s}$ to be the value function for the kicker who is the $j^{\text {th }}$ kicker to kick in Round $k$ when the state is $s=\left(s_{1}, s_{2}\right)$, where $s_{i}$ is the score for the team who kicks $i^{\text {th }}$ in Round $k$. Denote by $x_{i, j, s}$ the optimal kicking strategy for this kicker.

Round 3, Second Kick. Whether the team is currently even or behind, the optimal kicking strategy is always $x^{*}$, where $x^{*}$ is determined by the following first-order condition:

$$
P_{G}^{\prime}\left(x^{*}\right)\left[\frac{V_{W}-V_{L}}{2}+U_{G}\right]+P_{O}^{\prime}\left(x^{*}\right) U_{O}=0
$$

Round 3, First Kick. When the score is currently even $(s=(2,2),(1,1)$ or $s=(0,0))$, the value function for the team is $\frac{V_{W}+V_{L}}{2}$. When $s=(0,1)$, the value function for the kicker is

$$
\begin{aligned}
V_{3,1,(0,1)} & =P_{G}\left(x_{3,1,(0,1)}\right) P_{G}\left(x^{*}\right) V_{L}+P_{G}\left(x_{3,1,(0,1)}\right)\left(1-P_{G}\left(x^{*}\right)\right) \frac{V_{W}+V_{L}}{2}+\left(1-P_{G}\left(x_{3,1,(0,1)}\right)\right) V_{L} \\
& =\frac{V_{W}+V_{L}}{2}-\left[1-P_{G}\left(x_{3,1,(0,1)}\right)\left(1-P_{G}\left(x^{*}\right)\right)\right] \frac{V_{W}-V_{L}}{2}=\frac{V_{W}+V_{L}}{2}-\alpha_{3,1,(0,1)} \frac{V_{W}-V_{L}}{2}
\end{aligned}
$$

The optimal kicking strategy, $x_{3,1,(0,1)}$, satisfies the following first-order condition:

$$
P_{G}^{\prime}\left(x_{3,1,(0,1)}\right)\left[\left(1-P_{G}\left(x^{*}\right)\right) \frac{V_{W}-V_{L}}{2}+U_{G}\right]+P_{O}^{\prime}\left(x_{3,1,(0,1)}\right) U_{O}=0
$$

\footnotetext{
${ }^{38}$ We thank William Thomson for bringing the sports of petanque to our attention.

${ }^{39}$ See Article 16 of the world governing body of petanque FIPJP's official rulebook at http://fipjp.org/index. php/en/2015-05-10-11-11-42/petanque-rules retrieved on Feb 13, 2018.

${ }^{40}$ See Article 29 of the FIPJP's official rulebook.
} 
Similarly, we have $V_{3,1,(0,1)}=V_{3,1,(1,2)}$ and $x_{3,1,(0,1)}=x_{3,1,(1,2)}$. When $s=(1,0)$, the value function for the kicker is

$$
\begin{aligned}
V_{3,1,(1,0)} & =P_{G}\left(x_{3,1,(1,0)}\right) V_{W}+\left(1-P_{G}\left(x_{3,1,1,0)}\right)\right)\left[\left(1-P_{G}\left(x^{*}\right)\right) V_{W}+P_{G}\left(x^{*}\right) \frac{V_{W}+V_{L}}{2}\right] \\
& =\frac{V_{W}+V_{L}}{2}+\left[1-\left(1-P_{G}\left(x_{3,1,(1,0)}\right)\right) P_{G}\left(x^{*}\right)\right] \frac{V_{W}-V_{L}}{2}=\frac{V_{W}+V_{L}}{2}+\alpha_{3,1,(1,0)} \frac{V_{W}-V_{L}}{2}
\end{aligned}
$$

The optimal kicking strategy, $x_{3,1,(1,0)}$, satisfies the following first-order condition:

$$
P_{G}^{\prime}\left(x_{3,1,(1,0)}\right)\left[P_{G}\left(x^{*}\right) \frac{V_{W}-V_{L}}{2}+U_{G}\right]+P_{O}^{\prime}\left(x_{3,1,(1,0)}\right) U_{O}=0
$$

Similarly, we have $V_{3,1,(1,0)}=V_{3,1,(2,1)}$ and $x_{3,1,(1,0)}=x_{3,1,(2,1)}$.

Round 2, Second Kick. Denote by $\phi_{3}(s)$ the prob. that the first-kicking team in Round 2 kicks first in Round 3 when the state at the end of Round 2 is $s$. When $s=(0,0)$, the value function for the kicker is

$$
\begin{aligned}
V_{2,2,(0,0)} & =P_{G}\left(x_{2,2,(0,0)}\right)\left[\phi_{3}(0,1)\left(V_{W}+V_{L}-V_{3,1,(0,1)}\right)+\left(1-\phi_{3}(0,1)\right) V_{3,1,(1,0)}\right]+\left(1-P_{G}\left(x_{2,2,(0,0)}\right)\right) \frac{V_{W}+V_{L}}{2} \\
& =\frac{V_{W}+V_{L}}{2}+P_{G}\left(x_{2,2,(0,0)}\right) \alpha_{2,2,(0,0)} \frac{V_{W}-V_{L}}{2}
\end{aligned}
$$

where

$$
\alpha_{2,2,(0,0)}=\phi_{3}(0,1) \alpha_{3,1,(0,1)}+\left(1-\phi_{3}(0,1)\right) \alpha_{3,1,(1,0)}
$$

The optimal kicking strategy, $x_{2,2,(0,0)}$, satisfies the following first-order condition:

$$
P_{G}^{\prime}\left(x_{2,2,(0,0)}\right)\left[\alpha_{2,2,(0,0)} \frac{V_{W}-V_{L}}{2}+U_{G}\right]+P_{O}^{\prime}\left(x_{2,2,(0,0)}\right) U_{O}=0
$$

When $s=(1,0)$, the value function for the kicker is

$$
\begin{aligned}
V_{2,2,(1,0)} & =P_{G}\left(x_{2,2,(1,0)}\right) \frac{V_{W}+V_{L}}{2}+\left(1-P_{G}\left(x_{2,2,(1,0)}\right)\right)\left[\phi_{3}(1,0)\left(V_{W}+V_{L}-V_{3,1,(1,0)}\right)+\left(1-\phi_{3}(1,0)\right) V_{3,1,(0,1)}\right] \\
& =\frac{V_{W}+V_{L}}{2}-\left(1-P_{G}\left(x_{2,2,(1,0)}\right)\right) \alpha_{2,2,(1,0)} \frac{V_{W}-V_{L}}{2}
\end{aligned}
$$

where

$$
\alpha_{2,2,(1,0)}=\phi_{3}(1,0) \alpha_{3,1,(1,0)}+\left(1-\phi_{3}(1,0)\right) \alpha_{3,1,(0,1)} .
$$

The optimal kicking strategy, $x_{2,2,(1,0)}$, satisfies the following first-order condition:

$$
P_{G}^{\prime}\left(x_{2,2,(1,0)}\right)\left[\alpha_{2,2,(1,0)} \frac{V_{W}-V_{L}}{2}+U_{G}\right]+P_{O}^{\prime}\left(x_{2,2,(1,0)}\right) U_{O}=0
$$

When $s=(1,1)$, the value function for the kicker is

$$
\begin{aligned}
V_{2,2,(1,1)} & =P_{G}\left(x_{2,2,(1,1)}\right)\left[\phi_{3}(1,2)\left(V_{W}+V_{L}-V_{3,1,(1,2)}\right)+\left(1-\phi_{3}(1,2)\right) V_{3,1,(2,1)}\right]+\left(1-P_{G}\left(x_{2,2,(1,1)}\right)\right) \frac{V_{W}+V_{L}}{2} \\
& =\frac{V_{W}+V_{L}}{2}+P_{G}\left(x_{2,2,(1,1)}\right) \alpha_{2,2,(1,1)} \frac{V_{W}-V_{L}}{2}
\end{aligned}
$$


where

$$
\alpha_{2,2,(1,1)}=\phi_{3}(1,2) \alpha_{3,1,(1,2)}+\left(1-\phi_{3}(1,2)\right) \alpha_{3,1,(2,1)}
$$

The optimal kicking strategy, $x_{2,2,(1,1)}$, satisfies the following first-order condition:

$$
P_{G}^{\prime}\left(x_{2,2,(1,1)}\right)\left[\alpha_{2,2,(1,1)} \frac{V_{W}-V_{L}}{2}+U_{G}\right]+P_{O}^{\prime}\left(x_{2,2,(1,1)}\right) U_{O}=0
$$

When $s=(2,1)$, the value function for the kicker is

$$
\begin{aligned}
V_{2,2,(2,1)} & =P_{G}\left(x_{2,2,(2,1)}\right) \frac{V_{W}+V_{L}}{2}+\left(1-P_{G}\left(x_{2,2,(2,1)}\right)\right)\left[\phi_{3}(2,1)\left(V_{W}+V_{L}-V_{3,1,(2,1)}\right)+\left(1-\phi_{3}(2,1)\right) V_{3,1,(1,2)}\right] \\
& =\frac{V_{W}+V_{L}}{2}-\left(1-P_{G}\left(x_{2,2,(2,1)}\right)\right) \alpha_{2,2,(2,1)} \frac{V_{W}-V_{L}}{2}
\end{aligned}
$$

where

$$
\alpha_{2,2,(1,0)}=\phi_{3}(2,1) \alpha_{3,1,(2,1)}+\left(1-\phi_{3}(2,1)\right) \alpha_{3,1,(1,2)} .
$$

The optimal kicking strategy, $x_{2,2,(2,1)}$, satisfies the following first-order condition:

$$
P_{G}^{\prime}\left(x_{2,2,(2,1)}\right)\left[\alpha_{2,2,(1,0)} \frac{V_{W}-V_{L}}{2}+U_{G}\right]+P_{O}^{\prime}\left(x_{2,2,(2,1)}\right) U_{O}=0
$$

When $s=(0,1)$, the value function for the kicker is

$$
\begin{aligned}
V_{2,2,(0,1)} & =P_{G}\left(x_{2,2,(0,1)}\right) V_{W}+\left(1-P_{G}\left(x_{2,2,(0,1)}\right)\right)\left[\phi_{3}(0,1)\left(V_{W}+V_{L}-V_{3,1,(0,1)}\right)+\left(1-\phi_{3}(0,1)\right) V_{3,1,(1,0)}\right] \\
& =\frac{V_{W}+V_{L}}{2}+\alpha_{2,2,(0,1)} \frac{V_{W}-V_{L}}{2}
\end{aligned}
$$

where

$$
\alpha_{2,2,(0,1)}=P_{G}\left(x_{2,2,(0,1)}\right)+\left(1-P_{G}\left(x_{2,2,(0,1)}\right)\right)\left[\phi_{3}(0,1) \alpha_{3,1,(0,1)}+\left(1-\phi_{3}(0,1)\right) \alpha_{3,1,(1,0)}\right] .
$$

The optimal kicking strategy, $x_{2,2,(0,1)}$, satisfies the following first-order condition:

$$
P_{G}^{\prime}\left(x_{2,2,(0,1)}\right)\left\{\left[1-\left[\phi_{3}(0,1) \alpha_{3,1,(0,1)}+\left(1-\phi_{3}(0,1)\right) \alpha_{3,1,(1,0)}\right]\right] \frac{V_{W}-V_{L}}{2}+U_{G}\right\}+P_{O}^{\prime}\left(x_{2,2,(0,1)}\right) U_{O}=0
$$

When $s=(2,0)$, the value function for the kicker is

$$
\begin{aligned}
V_{2,2,(2,0)} & =P_{G}\left(x_{2,2,(2,0)}\right)\left[\phi_{3}(2,1)\left(V_{W}+V_{L}-V_{3,1,(2,1)}\right)+\left(1-\phi_{3}(2,1)\right) V_{3,1,(1,2)}\right]+\left(1-P_{G}\left(x_{2,2,(2,0)}\right)\right) V_{L} \\
& =\frac{V_{W}+V_{L}}{2}-\alpha_{2,2,(2,0)} \frac{V_{W}-V_{L}}{2}
\end{aligned}
$$

where

$$
\alpha_{2,2,(2,0)}=P_{G}\left(x_{2,2,(2,0)}\right)\left[\phi_{3}(2,1) \alpha_{3,1,(2,1)}+\left(1-\phi_{3}(2,1)\right) \alpha_{3,1,(1,2)}\right]+1-P_{G}\left(x_{2,2,(2,0)}\right) .
$$

The optimal kicking strategy, $x_{2,2,(2,0)}$, satisfies the following first-order condition:

$$
P_{G}^{\prime}\left(x_{2,2,(2,0)}\right)\left\{\left[1-\left[\phi_{3}(2,1) \alpha_{3,1,(2,1)}+\left(1-\phi_{3}(2,1)\right) \alpha_{3,1,(1,2)}\right]\right] \frac{V_{W}-V_{L}}{2}+U_{G}\right\}+P_{O}^{\prime}\left(x_{2,2,(2,0)}\right) U_{O}=0
$$


Round 2, First Kick. When $s=(0,0)$ or $s=(1,1)$, the value function for the team is $\frac{V_{W}+V_{L}}{2}$. When $s=(0,1)$, the value function for the kicker is

$$
\begin{aligned}
V_{2,1,(0,1)} & =P_{G}\left(x_{2,1,(0,1)}\right)\left(V_{W}+V_{L}-V_{2,2,(1,1)}\right)+\left(1-P_{G}\left(x_{2,1,(0,1)}\right)\right)\left(V_{W}+V_{L}-V_{2,2,(0,1)}\right) \\
& =\frac{V_{W}+V_{L}}{2}-\alpha_{2,1,(0,1)} \frac{V_{W}-V_{L}}{2}
\end{aligned}
$$

where

$$
\alpha_{2,1,(0,1)}=P_{G}\left(x_{2,1,(0,1)}\right) P_{G}\left(x_{2,2,(1,1)}\right) \alpha_{2,2,(1,1)}+\left(1-P_{G}\left(x_{2,1,(0,1)}\right)\right) \alpha_{2,2,(0,1)} .
$$

The optimal kicking strategy, $x_{2,1,(0,1)}$, satisfies the following first-order condition:

$$
P_{G}^{\prime}\left(x_{2,1,(0,1)}\right)\left\{\left[\alpha_{2,2,(0,1)}-P_{G}\left(x_{2,2,(1,1)}\right) \alpha_{2,2,(1,1)}\right] \frac{V_{W}-V_{L}}{2}+U_{G}\right\}+P_{O}^{\prime}\left(x_{2,1,(0,1)}\right) U_{O}=0
$$

When $s=(1,0)$, the value function for the kicker is

$$
\begin{aligned}
V_{2,1,(1,0)} & =P_{G}\left(x_{2,1,(1,0)}\right)\left(V_{W}+V_{L}-V_{2,2,(2,0)}\right)+\left(1-P_{G}\left(x_{2,1,(1,0)}\right)\right)\left(V_{W}+V_{L}-V_{2,2,(1,0)}\right) \\
& =\frac{V_{W}+V_{L}}{2}+\alpha_{2,1,(1,0)} \frac{V_{W}-V_{L}}{2}
\end{aligned}
$$

where

$$
\alpha_{2,1,(1,0)}=P_{G}\left(x_{2,1,(1,0)}\right) \alpha_{2,2,(2,0)}+\left(1-P_{G}\left(x_{2,1,(1,0)}\right)\right)\left(1-P_{G}\left(x_{2,2,(1,0)}\right)\right) \alpha_{2,2,(1,0)} .
$$

The optimal kicking strategy, $x_{2,1,(1,0)}$, satisfies the following first-order condition:

$$
P_{G}^{\prime}\left(x_{2,1,(1,0)}\right)\left\{\left[\alpha_{2,2,(2,0)}-\left(1-P_{G}\left(x_{2,2,(1,0)}\right)\right) \alpha_{2,2,(1,0)}\right] \frac{V_{W}-V_{L}}{2}+U_{G}\right\}+P_{O}^{\prime}\left(x_{2,1,(1,0)}\right) U_{O}=0
$$

Round 1, Second Kick. When $s=(0,0)$, the value function for the kicker is

$$
\begin{aligned}
V_{1,2,(0,0)} & =P_{G}\left(x_{1,2,(0,0)}\right)\left[\phi_{2}(0,1)\left(V_{W}+V_{L}-V_{2,1,(0,1)}\right)+\left(1-\phi_{2}(0,1)\right) V_{2,1,(1,0)}\right]+\left(1-P_{G}\left(x_{1,2,(0,0)}\right)\right) \frac{V_{W}+V_{L}}{2} \\
& =\frac{V_{W}+V_{L}}{2}+P_{G}\left(x_{1,2,(0,0)}\right) \alpha_{1,2,(0,0)} \frac{V_{W}-V_{L}}{2}
\end{aligned}
$$

where

$$
\alpha_{1,2,(0,0)}=\phi_{2}(0,1) \alpha_{2,1,(0,1)}+\left(1-\phi_{2}(0,1)\right) \alpha_{2,1,(1,0)} .
$$

The optimal kicking strategy, $x_{1,2,(0,0)}$, satisfies the following first-order condition:

$$
P_{G}^{\prime}\left(x_{1,2,(0,0)}\right)\left[\alpha_{1,2,(0,0)} \frac{V_{W}-V_{L}}{2}+U_{G}\right]+P_{O}^{\prime}\left(x_{1,2,(0,0)}\right) U_{O}=0 .
$$

When $s=(1,0)$, the value function for the kicker is

$$
\begin{aligned}
V_{1,2,(1,0)} & =P_{G}\left(x_{1,2,(1,0)}\right) \frac{V_{W}+V_{L}}{2}+\left(1-P_{G}\left(x_{1,2,(1,0)}\right)\right)\left[\phi_{2}(1,0)\left(V_{W}+V_{L}-V_{2,1,(1,0)}\right)\right. \\
& \left.+\left(1-\phi_{2}(1,0)\right) V_{2,1,(0,1)}\right] \\
& =\frac{V_{W}+V_{L}}{2}-\left(1-P_{G}\left(x_{1,2,(1,0)}\right)\right) \alpha_{1,2,(1,0)} \frac{V_{W}-V_{L}}{2},
\end{aligned}
$$


where

$$
\alpha_{1,2,(1,0)}=\phi_{2}(1,0) \alpha_{2,1,(1,0)}+\left(1-\phi_{2}(1,0)\right) \alpha_{2,1,(0,1)}
$$

The optimal kicking strategy, $x_{1,2,(1,0)}$, satisfies the following first-order condition:

$$
P_{G}^{\prime}\left(x_{1,2,(1,0)}\right)\left[\alpha_{1,2,(1,0)} \frac{V_{W}-V_{L}}{2}+U_{G}\right]+P_{O}^{\prime}\left(x_{1,2,(1,0)}\right) U_{O}=0 .
$$

Round 1, First Kick. The value function for the kicker is

$$
\begin{aligned}
V_{1,1,(0,0)} & =P_{G}\left(x_{1,1,(0,0)}\right)\left[V_{W}+V_{L}-V_{1,2,(1,0)}\right]+\left(1-P_{G}\left(x_{1,1,(0,0)}\right)\right)\left[V_{W}+V_{L}-V_{1,2,(0,0)}\right] \\
& =\frac{V_{W}+V_{L}}{2}+\left[P_{G}\left(x_{1,1,(0,0)}\right)\left(1-P_{G}\left(x_{1,2,(1,0)}\right)\right) \alpha_{1,2,(1,0)}\right. \\
& \left.-\left(1-P_{G}\left(x_{1,1,(0,0)}\right)\right) P_{G}\left(x_{1,2,(0,0)}\right) \alpha_{1,2,(0,0)}\right] \frac{V_{W}-V_{L}}{2}
\end{aligned}
$$

The optimal kicking strategy, $x_{1,1,(0,0)}$, satisfies the following first-order condition:

$P_{G}^{\prime}\left(x_{1,1,(0,0)}\right)\left[\left[\left(1-P_{G}\left(x_{1,2,(1,0)}\right)\right) \alpha_{1,2,(1,0)}+P_{G}\left(x_{1,2,(0,0)}\right) \alpha_{1,2,(0,0)}\right] \frac{V_{W}-V_{L}}{2}+U_{G}\right]+P_{O}^{\prime}\left(x_{1,1,(0,0)}\right) U_{O}=0$

Therefore

$$
x_{1,1,(0,0)} \gtreqless x_{1,2,(0,0)} \Longleftrightarrow\left(1-P_{G}\left(x_{1,2,(1,0)}\right)\right) \alpha_{1,2,(1,0)} \gtreqless P_{G}\left(x_{1,2,(0,0)}\right) \alpha_{1,2,(0,0)}
$$

On the other hand, we have

$$
\begin{aligned}
V_{1,1,(0,0)} & =\frac{V_{W}+V_{L}}{2} \Longleftrightarrow P_{G}\left(x_{1,1,(0,0)}\right)\left(1-P_{G}\left(x_{1,2,(1,0)}\right)\right) \alpha_{1,2,(1,0)}=\left(1-P_{G}\left(x_{1,1,(0,0)}\right)\right) P_{G}\left(x_{1,2,(0,0)}\right) \alpha_{1,2,(0,0)} \\
& \Longleftrightarrow\left(1-P_{G}\left(x_{1,2,(1,0)}\right)\right) \alpha_{1,2,(1,0)}=\left(1-P_{G}\left(x_{1,1,(0,0)}\right)\right) \alpha_{1,2,(0,0)}
\end{aligned}
$$

The condition holds if $\phi_{2}(1,0)+\phi_{2}(0,1)=1$.

\section{H First-Mover Advantage: A Refinement}

Here, we address the question as to which state-symmetric equilibrium is more likely to be observed when there are multiple state-symmetric equilibria in ABAB. To that end, we use a selection criterion. Suppose there are multiple state-symmetric equilibria. Let the state-symmetric equilibrium with $\left(x^{*}, y_{E}^{*}, y_{B}^{*}\right)$ be the one with highest $x$, i.e., the intended spot by Team 1's kickers is the closest to the goal-optimal spot among all state-symmetric equilibria. We will refer to this equilibrium as the most aggressive equilibrium for Team 1 for the following reason: As $x^{*}=y_{E}^{*}>y_{B}^{*}$, we have the winning probability of Team $1, \alpha=\frac{1-P_{G}\left(y_{B}^{*}\right)}{2-P_{G}\left(y_{E}^{*}\right)-P_{G}\left(y_{B}^{*}\right)}>\frac{1}{2}$ by Equation 13; and moreover, such a winning probability for Team 1 is the highest among all state-symmetric equilibria. As a result, Team 1's kickers can collectively enforce the most aggressive kicking equilibrium for their team and win more often, in which the first kicker can set the tone of aggressiveness for his team. Being the first mover, if Team 1 can credibly signal Team 2 that they are indeed playing this most aggressive 
equilibrium, this would be the most beneficial for Team 1. In this case, we can use such a signaling through beliefs in the state-symmetric equilibrium to obtain a refinement. For example, if $\sigma_{x^{*}}$, the probability density function of the ball reaching a particular spot on the goal line when it is aimed at $x^{*}$ has the support set $\left[x^{*}-\epsilon_{x^{*}}, x^{*}+\overline{\epsilon_{x^{*}}}\right]$. Suppose that this support is disjoint from such support sets of other equilibria. Then, whenever Team 2 kickers observe a kick spot in $\sigma_{x^{*}}$ 's support, they can credibly deduce that indeed Team 1 is playing this aggressive equilibrium. Hence, the beliefs of Team 2's kickers in information sets that are never reached in a state-symmetric equilibrium can be fine-tuned so that less aggressive equilibria can be eliminated.

Definition 1 (Refinement Criterion) If the most aggressive state-symmetric equilibrium for Team 1 involves aiming at $x^{*}$ for each kicker, and the possible spots that the ball can go under $x^{*}$ (as determined by the support of $\sigma_{x^{*}},\left[x^{*}-\underline{\epsilon}_{x^{*}}, x^{*}+\bar{\epsilon}_{x^{*}}\right]$ ) are different from any of the spots that the ball can go under all other state-symmetric equilibria, then Team 1 can credibly enforce the most aggressive state-symmetric equilibrium.

Hence, we get the following corollary:

Corollary 3 (Team 1 wins more often) If the state-symmetric equilibria can be refined, then Team 1, the team that kicks first, wins with a higher probability than Team 2 in the sudden-death rounds of $A B A B$.

Hence, in our analysis with equally-skilled players and goalies, ABAB is biased toward the first-moving team and further multiple equilibria certainly exist. Indeed, empirically as well, these multiple equilibria and the overall first-mover advantage are evident. The relative frequency figures regarding the winning probability of the teams that kick first vary significantly across tournaments throughout the world (see Figure A.1). 